\title{
Motobamide, an Antitrypanosomal Cyclic Peptide from a Leptolyngbya sp. Marine
}

\section{Cyanobacterium.}

Hiroki Takahashi, ${ }^{\dagger}$ Arihiro Iwasaki,${ }^{\dagger}$ Naoaki Kurisawa, ${ }^{\dagger}$ Ryota Suzuki, ${ }^{\ddagger}$ Ghulam Jeelani, ${ }^{\S}$ Teruhiko Matsubara,^ Toshinori Sato,^ Tomoyoshi Nozaki, ${ }^{\S}$ Kiyotake Suenaga $*, \dagger$

'Department of Chemistry, Faculty of Science and Technology, Keio University, 3-14-1 Hiyoshi, Kohoku-ku, Yokohama, Kanagawa 223-8522, Japan

*S\&E Simulation, 4-15-2 Sasage, Kohnan-ku, Yokohama, Kanagawa 234-0052, Japan

$\S$ Department of Biomedical Chemistry, Graduate School of Medicine, The University of Tokyo, Tokyo, 7-3-1 Hongo, Bunkyo-ku, Tokyo 113-0033, Japan.

${ }^{\wedge}$ Department of Biosciences and Informatics, Faculty of Science and Technology, Keio University, 3-14-1 Hiyoshi, Kohoku-ku, Yokohama, Kanagawa 223-8522, Japan

\section{Contents}

NMR data of motobamide (1)

Analyses of the stereochemistry of amino acids in motobamide (1)

S 9-11

$\mathrm{MS}^{2}$ and $\mathrm{MS}^{3}$ spectra of motobamide (1)

S 12

Phylogenetic tree of the cyanobacterial sample

Detailed results of the computational chemistry 
Figure $\mathrm{S} 1 .{ }^{1} \mathrm{H}$ NMR (400 MHz, $\mathrm{CD}_{3} \mathrm{OD}$ ) spectrum of motobamide (1)

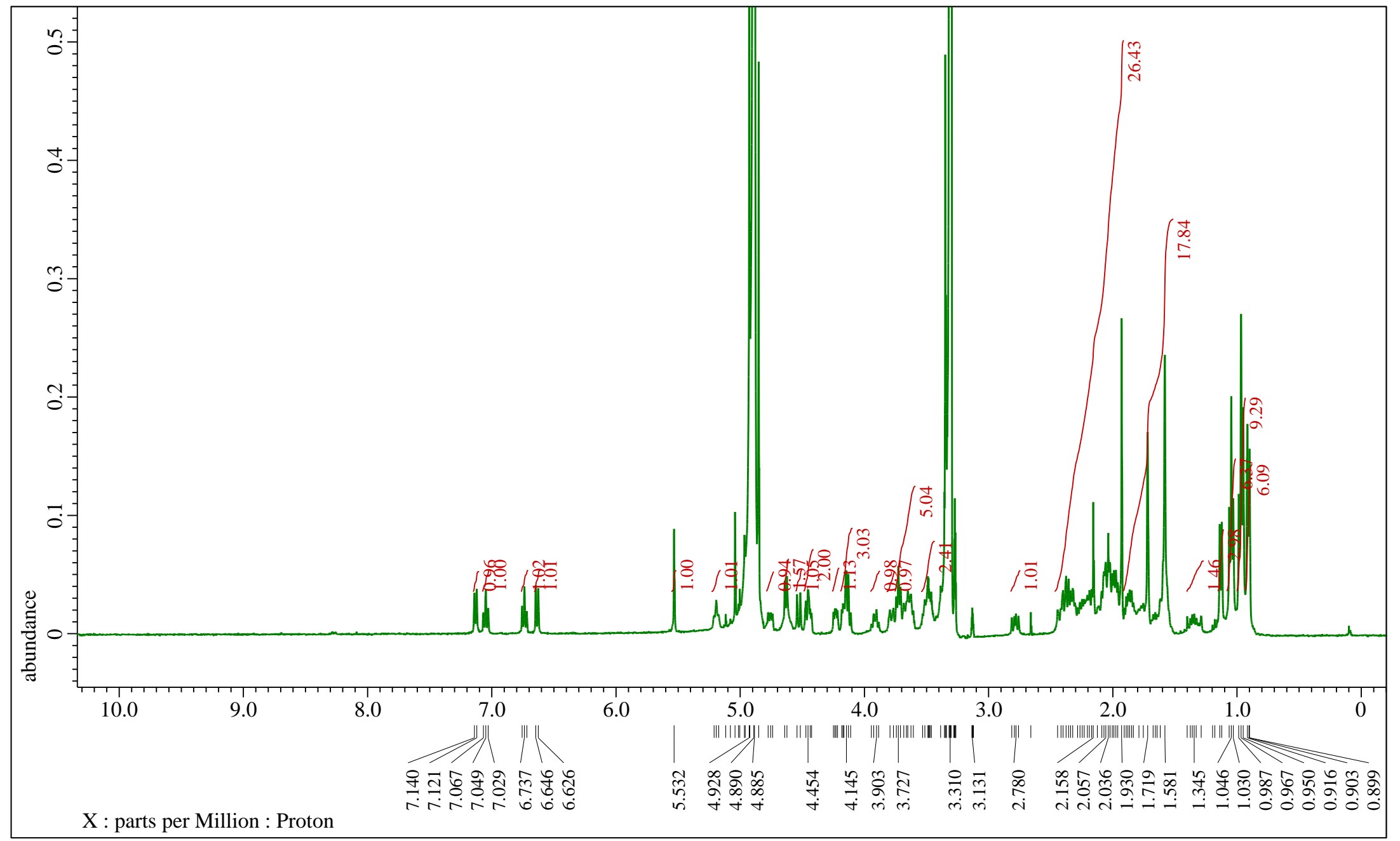


Figure $\mathrm{S} 2 .{ }^{13} \mathrm{C}$ NMR (100 MHz, $\left.\mathrm{CD}_{3} \mathrm{OD}\right)$ spectrum of motobamide (1)

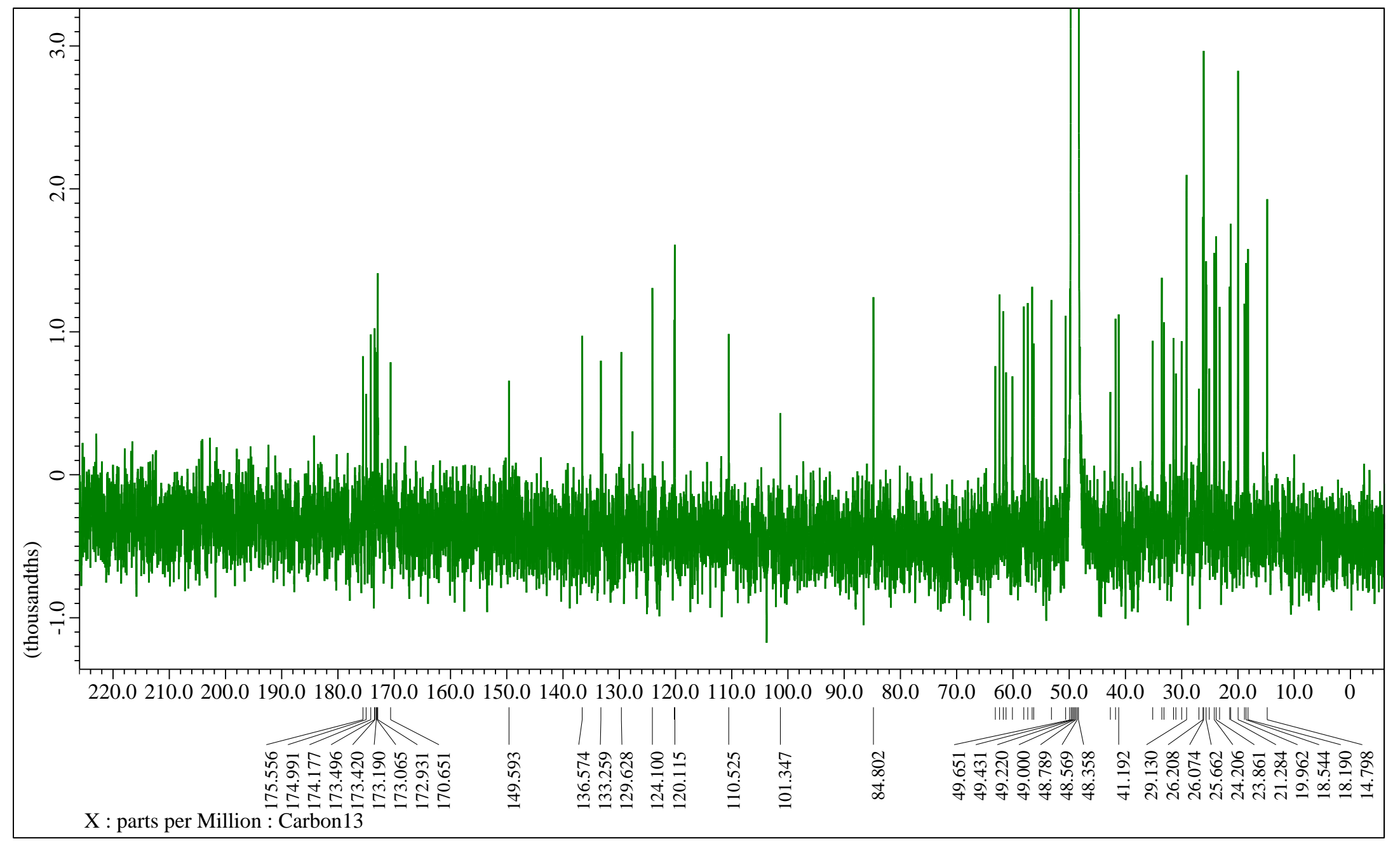


Figure S3. COSY (400 MHz, $\mathrm{CD}_{3} \mathrm{OD}$ ) spectrum of motobamide (1)

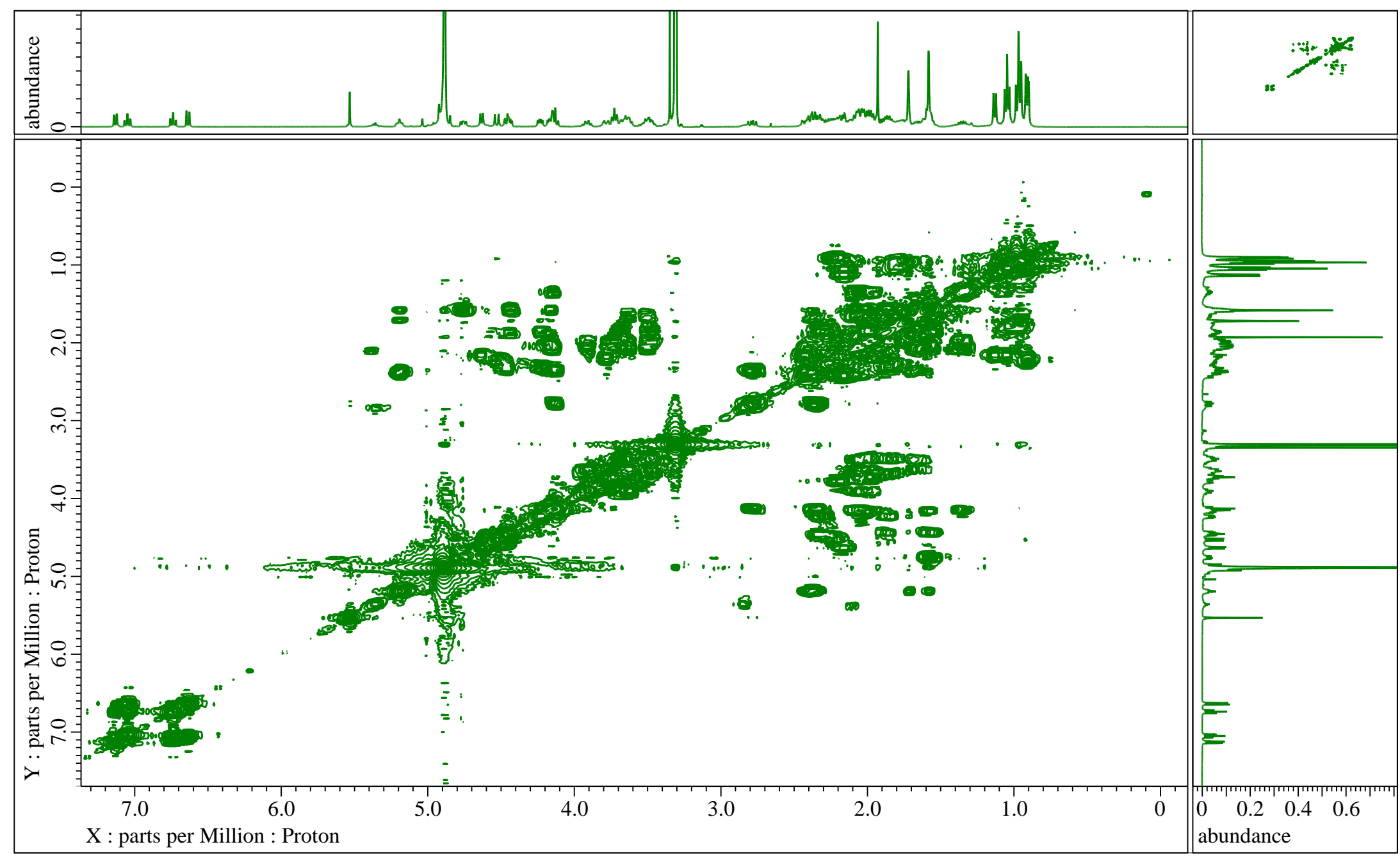


Figure $\mathrm{S} 4 . \mathrm{HMQC}\left(400 \mathrm{MHz}, \mathrm{CD}_{3} \mathrm{OD}\right)$ spectrum of motobamide (1)

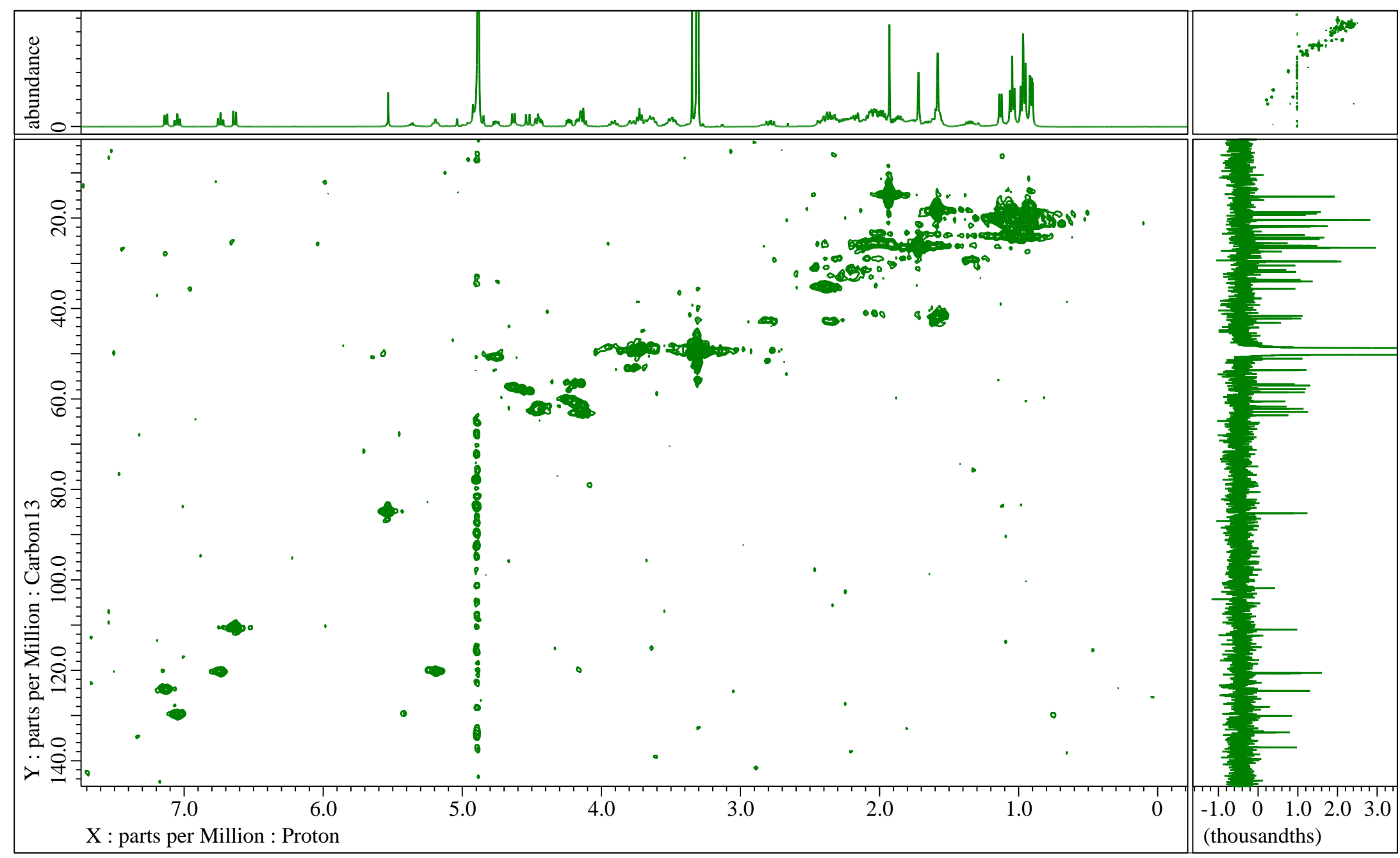


Figure $\mathrm{S} 5 . \mathrm{HMBC}\left(400 \mathrm{MHz}, \mathrm{CD}_{3} \mathrm{OD}\right)$ spectrum of motobamide (1)

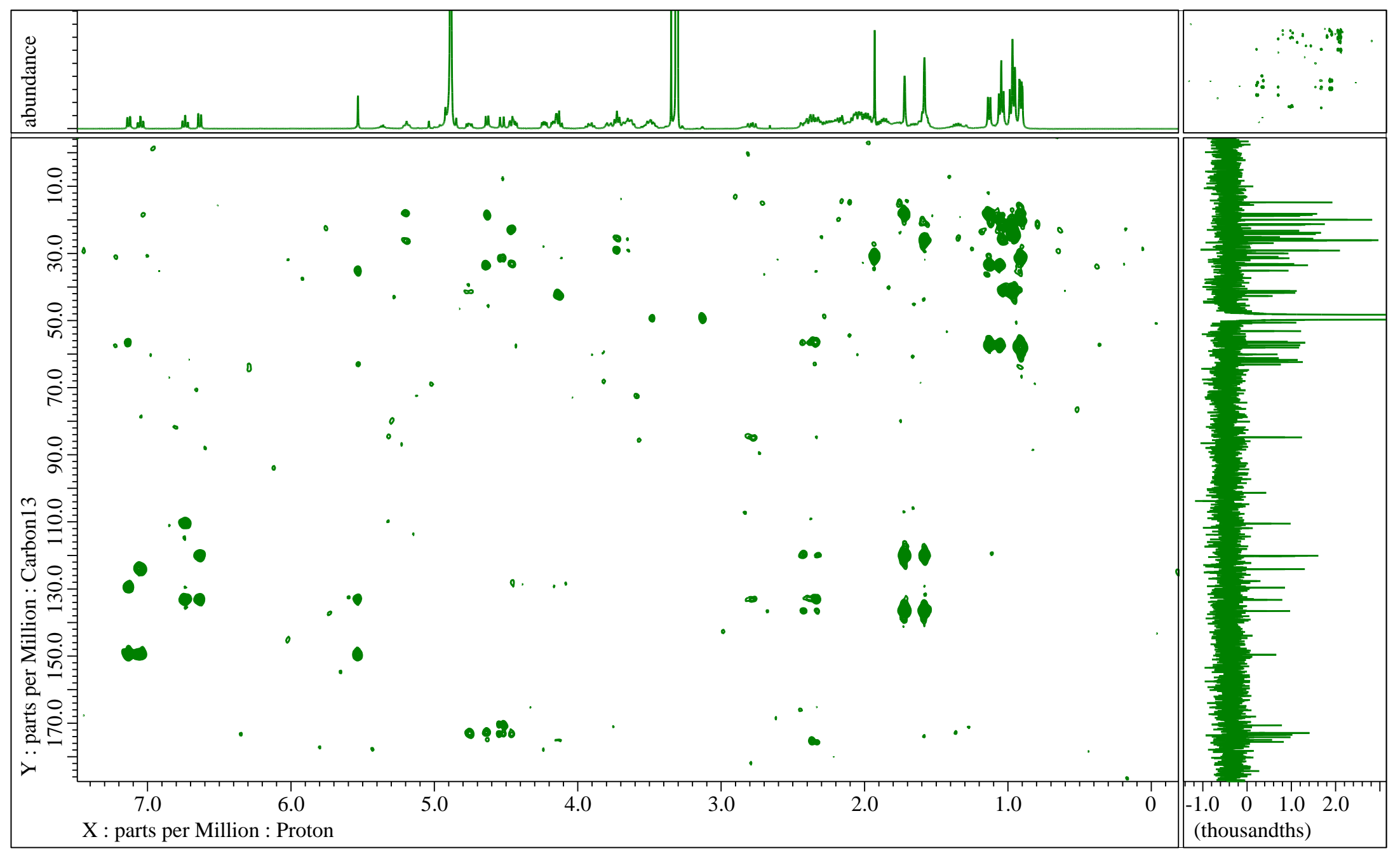


Figure S6. NOESY (400 MHz, $\mathrm{CD}_{3} \mathrm{OD}$ ) spectrum of motobamide (1)




Figure S7. TOCSY (400 MHz, $\mathrm{CD}_{3} \mathrm{OD}$ ) spectrum of motobamide (1)






\section{Chiral HPLC analysis}

Determination of the absolute configuration of the $\alpha$-amino acids in motobamide (1)

Pro: column, DAICEL CHIRALPAK $(\mathrm{MA}+)(\phi 4.6 \times 50 \mathrm{~mm})$; flow rate $1.0 \mathrm{~mL} / \mathrm{min}$; detection at $254 \mathrm{~nm}$; solvent, $2.0 \mathrm{mM} \mathrm{CuSO}_{4}$.

$t_{\mathrm{R}}$ (min): Authentic samples: D-Pro (2.9), L-Pro (5.8)
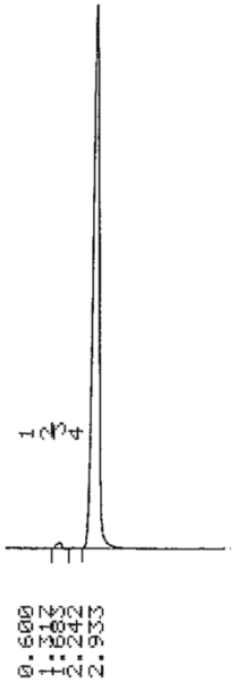

D-Pro
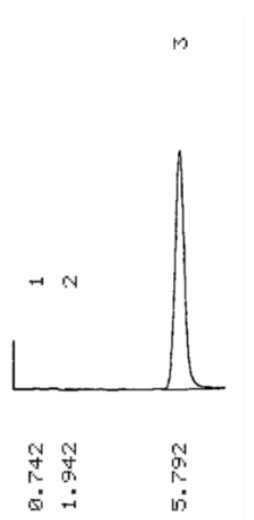

L-Pro

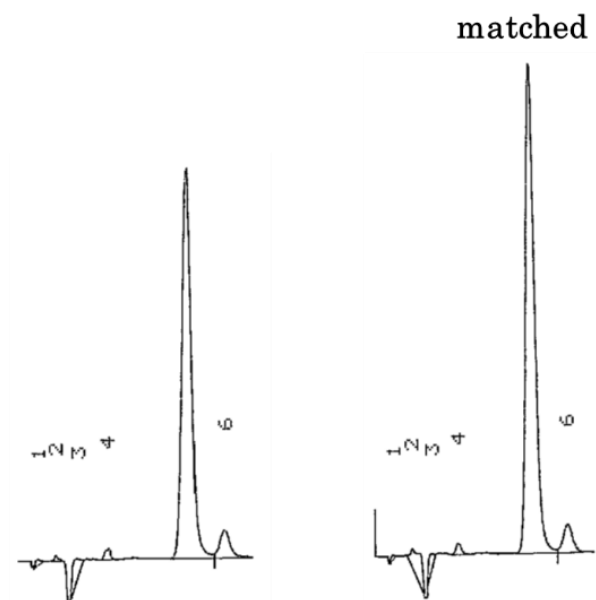

Pro from 1
Pro from 1 L-Pro

Val: column, DAICEL CHIRALPAK $(\mathrm{MA}+)(\phi 4.6 \times 50 \mathrm{~mm})$; flow rate $1.0 \mathrm{~mL} / \mathrm{min}$; detection at $254 \mathrm{~nm}$; solvent, $2.0 \mathrm{mM} \mathrm{CuSO}_{4}$.

$t_{\mathrm{R}}$ (min): Authentic samples: D-Val (3.8), L-Val (6.7)

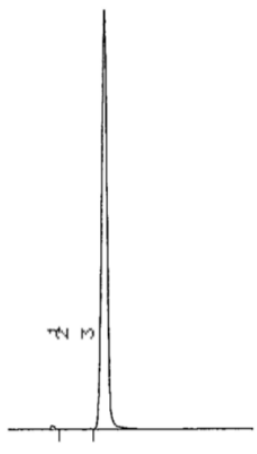

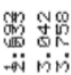

D-Val

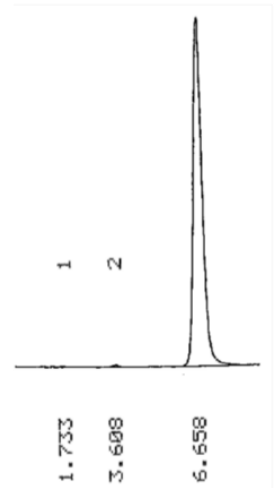

L-Val

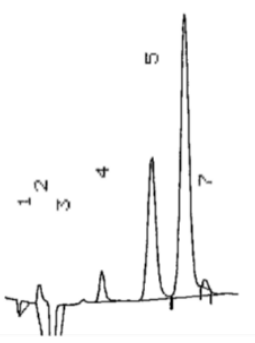

Val from 1



Val from 1 L-Val 
Met: column, DAICEL CHIRALPAK (MA+) $(\phi 4.6 \times 50 \mathrm{~mm})$; flow rate $1.0 \mathrm{~mL} / \mathrm{min}$; detection at $254 \mathrm{~nm}$; solvent, $2.0 \mathrm{mM} \mathrm{CuSO}_{4}$.

$t_{\mathrm{R}}$ (min): Authentic samples: D-Met (6.8), L-Met (11.2)

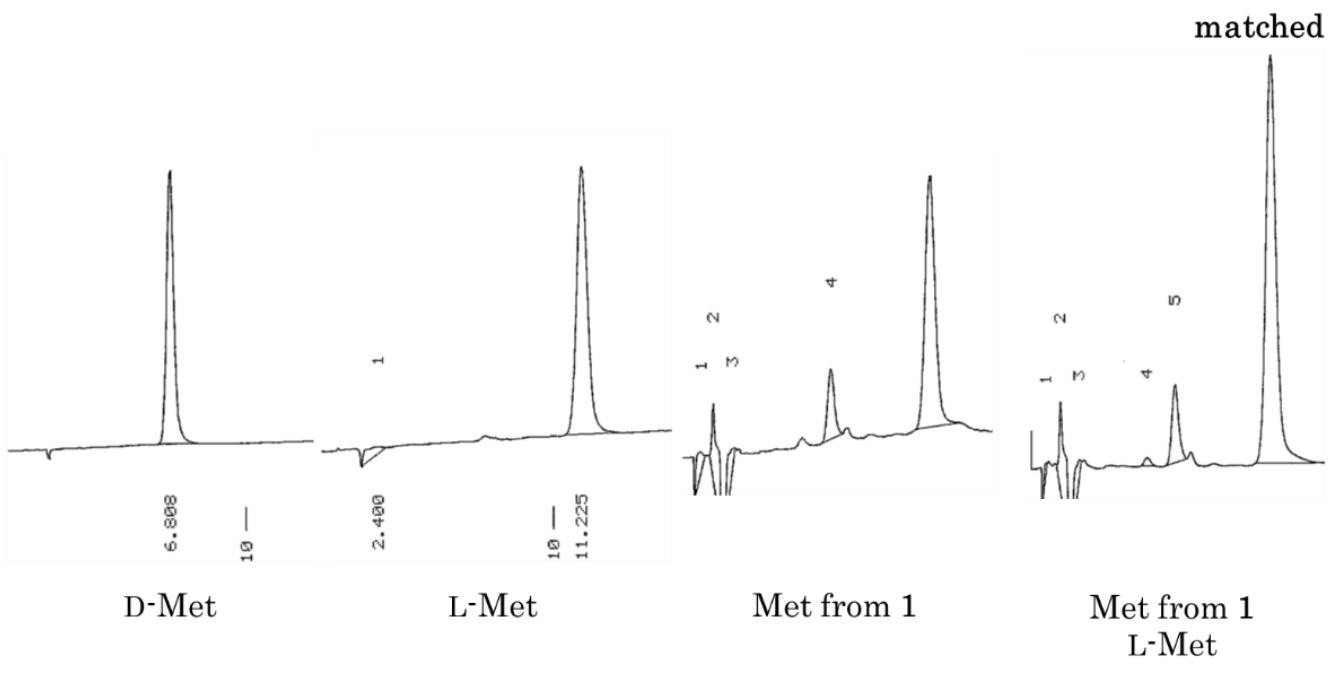

Leu: column, DAICEL CHIRALPAK $(\mathrm{MA}+)(\phi 4.6 \times 50 \mathrm{~mm})$; flow rate $1.0 \mathrm{~mL} / \mathrm{min}$; detection at $254 \mathrm{~nm}$; solvent, $2.0 \mathrm{mM} \mathrm{CuSO}_{4}$.

$t_{\mathrm{R}}$ (min): Authentic samples: D-Leu (8.1), L-Leu (15.3)

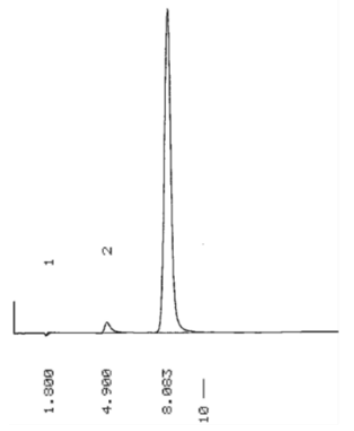

D-Leu

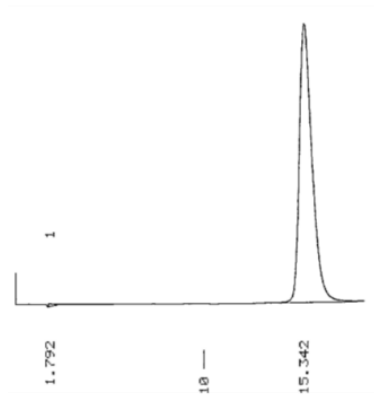

L-Leu

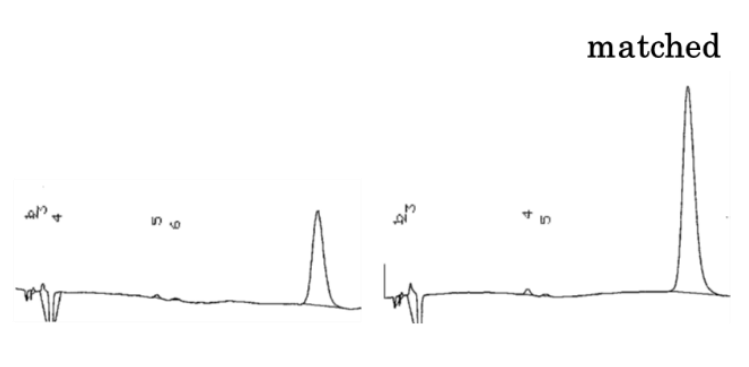

Leu from 1
Leu from 1

L-Leu 
Chiral HPLC analysis of Trp from Prenyl Trp of motobamide (1)

Trp from Prenyl Trp of HT-1-110-4 (1): column, DAICEL CHIRALPAK (MA+) ( $\phi 4.6 \times$ $50 \mathrm{~mm}$ ); flow rate $1.0 \mathrm{~mL} / \mathrm{min}$; detection at $254 \mathrm{~nm}$; solvent, $15 \% \mathrm{MeCN} 2.0 \mathrm{mM}$ $\mathrm{CuSO}_{4}$

$t_{\mathrm{R}}$ (min): Authentic samples: D-Trp (8.8), L-Trp (10.8)

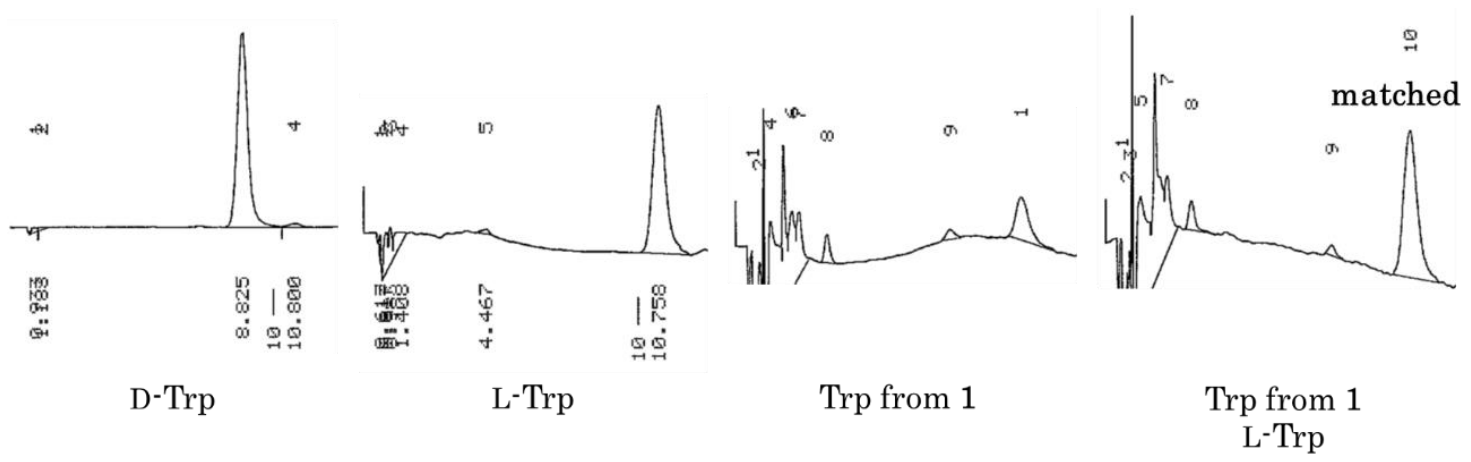




\section{$\mathrm{MS}^{2}$ and $\mathrm{MS}^{3}$ spectra of motobamide (1)}

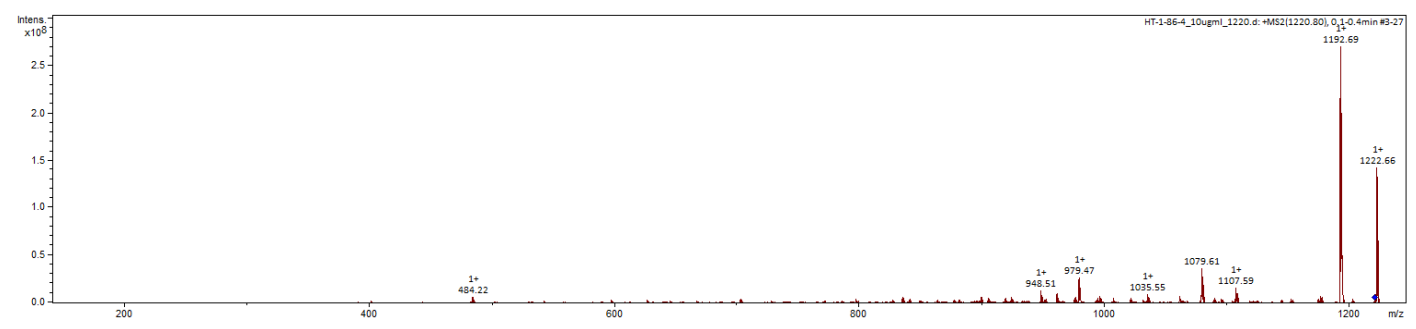

MS2 spectrum of motobamide (1) (precursor, $\mathrm{m} / \mathrm{z} 1221[\mathrm{M}+\mathrm{Na}]+$ )



$\mathrm{MS}^{3}$ spectrum of a fragment $\left(\mathrm{m} / z 1193[\mathrm{M}+\mathrm{Na}]^{+}\right)$from motobamide (1)

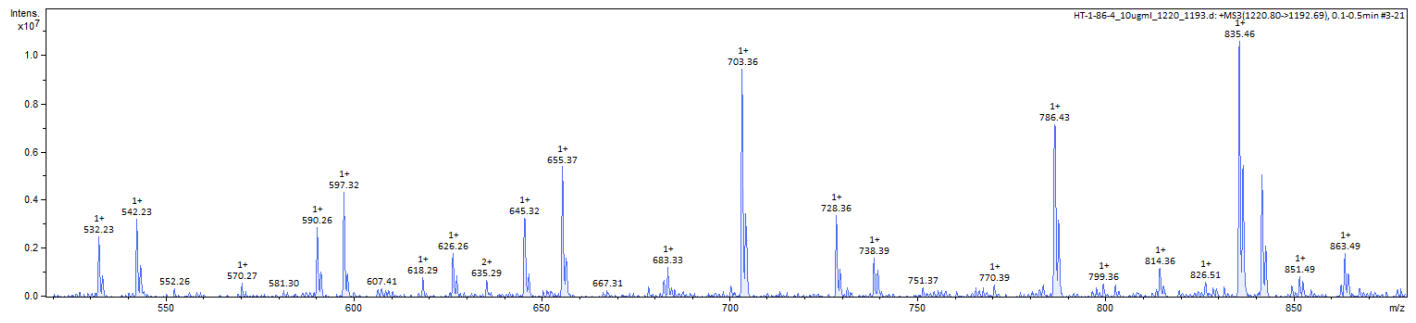

Magnified spectrum of

$\mathrm{MS}^{3}$ spectrum of a fragment $\left(\mathrm{m} / z 1193[\mathrm{M}+\mathrm{Na}]^{+}\right)$from motobamide (1) 


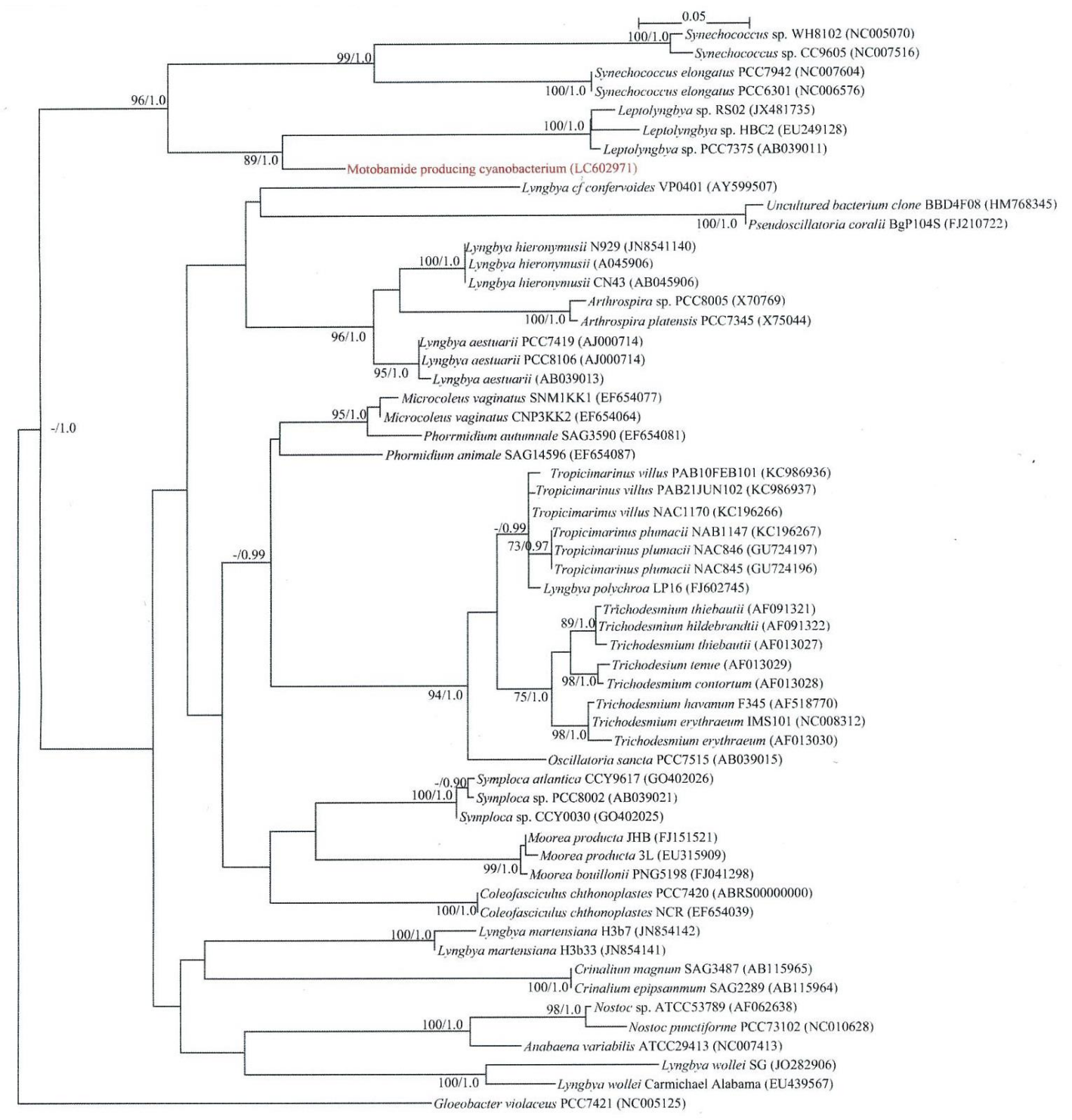

Figure S8. Phylogenetic tree inferred from 16S rDNA sequences using the ML method. The phylogeny is rooted with Gloeobacter violaceus PCC 7421. The numbers at the nodes indicate bootstrap values (values $<70 \%$ are not shown) and posterior probability (values $<0.9$ are not shown) for the ML/Bayesian inference. 
Table S1. Energy and Boltzmann population of optimized conformers of (8S, 10S, 16S)2 which has $>1 \%$ Boltzmann population.

\begin{tabular}{|c|l|r|r|}
\hline Number & Conformer & Energy (Hartree) & Boltzmann Population at 298.15 K \\
\hline $\mathbf{1}$ & SSS1 & -1053.068783 & $50.76 \%$ \\
\hline $\mathbf{2}$ & SSS2 & -1053.066591 & $4.97 \%$ \\
\hline $\mathbf{3}$ & SSS4 & -1053.066805 & $6.24 \%$ \\
\hline $\mathbf{4}$ & SSS8 & -1053.066848 & $6.53 \%$ \\
\hline $\mathbf{5}$ & SSS11 & -1053.065114 & $1.04 \%$ \\
\hline $\mathbf{6}$ & SSS12 & -1053.068175 & $26.66 \%$ \\
\hline $\mathbf{7}$ & SSS15 & -1053.065668 & $1.87 \%$ \\
\hline
\end{tabular}

Table S2. Energy and Boltzmann population of optimized conformers of $(8 S, 10 R, 16 R)$ 2 which has $>1 \%$ Boltzmann population.

\begin{tabular}{|r|l|r|r|}
\hline Number & Conformer & Energy (Hartree) & Boltzmann Population at 298.15 K \\
\hline $\mathbf{1}$ & SRR1 & -1053.069065 & $7.13 \%$ \\
\hline $\mathbf{2}$ & SRR2 & -1053.069801 & $15.55 \%$ \\
\hline $\mathbf{3}$ & SRR3 & -1053.069023 & $6.82 \%$ \\
\hline $\mathbf{4}$ & SRR4 & -1053.068693 & $4.81 \%$ \\
\hline $\mathbf{5}$ & SRR5 & -1053.068193 & $2.83 \%$ \\
\hline $\mathbf{6}$ & SRR6 & -1053.069123 & $7.58 \%$ \\
\hline $\mathbf{7}$ & SRR7 & -1053.068328 & $3.26 \%$ \\
\hline $\mathbf{8}$ & SRR8 & -1053.069363 & $9.77 \%$ \\
\hline $\mathbf{9}$ & SRR11 & -1053.069553 & $11.96 \%$ \\
\hline $\mathbf{1 0}$ & SRR12 & -1053.069724 & $14.33 \%$ \\
\hline $\mathbf{1 1}$ & SRR13 & -1053.068693 & $4.80 \%$ \\
\hline $\mathbf{1 2}$ & SRR14 & -1053.067989 & $2.28 \%$ \\
\hline $\mathbf{1 3}$ & SRR19 & -1053.067909 & $2.09 \%$ \\
\hline $\mathbf{1 4}$ & SRR20 & -1053.068536 & $4.07 \%$ \\
\hline $\mathbf{1 5}$ & SRR22 & -1053.068157 & $2.72 \%$ \\
\hline & & & \\
\hline
\end{tabular}


Structure and geometry of the optimized $(8 S, 10 S, 16 S)-2$ conformers with $>1 \%$ Boltzmann population

\section{SSS1}

$\begin{array}{lll}0 & 1\end{array}$

$\mathrm{C}$

C 11 r2

C $2 \begin{array}{llll}\text { r } 3 & 1 & \text { a3 }\end{array}$

C $\begin{array}{llllll}3 & \mathrm{r} 4 & 2 & \mathrm{a} 4 & 1 & \mathrm{~d} 4\end{array}$

C $\quad 4 \begin{array}{lllll}\text { r5 } & 3 & \text { a5 } & 2 & \text { d } 5\end{array}$

C $5 \begin{array}{llllll}5 & \mathrm{r} 6 & 4 & \mathrm{a} 6 & 3 & \mathrm{~d} 6\end{array}$

$\begin{array}{llllllll}\mathrm{C} & 3 & \mathrm{r} 7 & 2 & \text { a7 } & 1 & \mathrm{~d} 7\end{array}$

$\begin{array}{llllllll}\mathrm{C} & 7 & \mathrm{r} 8 & 3 & \mathrm{a} 8 & 2 & \mathrm{~d} 8\end{array}$

$\begin{array}{lllllll}\mathrm{N} & 2 & \mathrm{r} 9 & 1 & \mathrm{a} 9 & 3 & \mathrm{~d} 9\end{array}$

$\begin{array}{lllllll}\mathrm{N} & 8 & \mathrm{r} 10 & 7 & \mathrm{a} 10 & 3 & \mathrm{~d} 10\end{array}$

$\begin{array}{lllllll}\text { C } & 10 & \text { r11 } & 8 & \text { a11 } & 7 & \text { d } 11\end{array}$

$\begin{array}{lllllll}\text { C } & 11 & \text { r12 } & 10 & \text { a12 } & 8 & \text { d12 }\end{array}$

$\begin{array}{lllllll}\text { C } & 11 & \text { r13 } & 10 & \text { a13 } & 8 & \text { d13 }\end{array}$

$\begin{array}{lllllll}\text { C } & 10 & \text { r14 } & 8 & \text { a14 } & 7 & \text { d14 }\end{array}$

$\begin{array}{lllllll}\text { C } & 7 & \text { r15 } & 3 & \text { a15 } & 2 & \text { d15 }\end{array}$

$\begin{array}{lllllll}\text { C } & 15 & \text { r16 } & 7 & \text { a16 } & 3 & \text { d16 }\end{array}$

$\begin{array}{lllllll}\text { C } & 16 & \text { r17 } & 15 & \text { a17 } & 7 & \text { d17 }\end{array}$

$\begin{array}{lllllll}\text { C } & 17 & \text { r18 } & 16 & \text { a18 } & 15 & \text { d18 }\end{array}$

$\begin{array}{lllllll}\text { C } & 17 & \text { r19 } & 16 & \text { a19 } & 15 & \text { d19 }\end{array}$

$\begin{array}{lllllll}\mathrm{N} & 13 & \mathrm{r} 20 & 11 & \mathrm{a} 20 & 10 & \mathrm{~d} 20\end{array}$

$\begin{array}{lllllll}\mathrm{O} & 13 & \mathrm{r} 21 & 11 & \mathrm{a} 21 & 10 & \mathrm{~d} 21\end{array}$

$\begin{array}{lllllll}\mathrm{C} & 14 & \mathrm{r} 22 & 10 & \mathrm{a} 22 & 8 & \mathrm{~d} 22\end{array}$

$\begin{array}{lllllll}\mathrm{O} & 14 & \text { r23 } & 10 & \text { a23 } & 8 & \text { d } 23\end{array}$

$\begin{array}{lllllll}\text { C } & 20 & \text { r24 } & 13 & \text { a24 } & 11 & \text { d24 }\end{array}$

$\begin{array}{lllllll}\mathrm{H} & 11 & \mathrm{r} 25 & 10 & \mathrm{a} 25 & 8 & \mathrm{~d} 25\end{array}$

$\begin{array}{lllllll}\mathrm{H} & 8 & \mathrm{r} 26 & 7 & \mathrm{a} 26 & 3 & \mathrm{~d} 26\end{array}$

$\begin{array}{lllllll}\mathrm{H} & 1 & \mathrm{r} 27 & 2 & \mathrm{a} 27 & 3 & \mathrm{~d} 27\end{array}$

$\begin{array}{lllllll}\mathrm{H} & 4 & \mathrm{r} 28 & 3 & \mathrm{a} 28 & 2 & \mathrm{~d} 28\end{array}$

$\begin{array}{lllllll}\mathrm{H} & 5 & \mathrm{r} 29 & 4 & \mathrm{a} 29 & 3 & \mathrm{~d} 29\end{array}$

$\begin{array}{lllllll}\mathrm{H} & 6 & \text { r30 } & 5 & \text { a } 30 & 4 & \text { d } 30\end{array}$

$\begin{array}{lllllll}\mathrm{H} & 9 & \mathrm{r} 31 & 2 & \mathrm{a} 31 & 1 & \mathrm{~d} 31\end{array}$

$\begin{array}{lllllll}\mathrm{H} & 12 & \text { r32 } & 11 & \text { a32 } & 10 & \text { d } 32\end{array}$ 


$$
\begin{aligned}
& \begin{array}{lllllll}
\mathrm{H} & 12 & \text { r33 } & 11 & \text { a33 } & 10 & \text { d33 }
\end{array} \\
& \begin{array}{lllllll}
\mathrm{H} & 15 & \mathrm{r} 34 & 7 & \mathrm{a} 34 & 3 & \mathrm{~d} 34
\end{array} \\
& \begin{array}{lllllll}
\mathrm{H} & 15 & \mathrm{r} 35 & 7 & \mathrm{a} 35 & 3 & \mathrm{~d} 35
\end{array} \\
& \begin{array}{lllllll}
\mathrm{H} & 16 & \mathrm{r} 36 & 15 & \mathrm{a} 36 & 7 & \mathrm{~d} 36
\end{array} \\
& \begin{array}{lllllll}
\mathrm{H} & 18 & \mathrm{r} 37 & 17 & \mathrm{a} 37 & 16 & \mathrm{~d} 37
\end{array} \\
& \begin{array}{lllllll}
\mathrm{H} & 18 & \text { r38 } & 17 & \text { a38 } & 16 & \text { d } 38
\end{array} \\
& \begin{array}{lllllll}
\mathrm{H} & 18 & \mathrm{r} 39 & 17 & \mathrm{a} 39 & 16 & \mathrm{~d} 39
\end{array} \\
& \begin{array}{lllllll}
\mathrm{H} & 19 & \mathrm{r} 40 & 17 & \mathrm{a} 40 & 16 & \mathrm{~d} 40
\end{array} \\
& \begin{array}{lllllll}
\mathrm{H} & 19 & \mathrm{r} 41 & 17 & \mathrm{a} 41 & 16 & \mathrm{~d} 41
\end{array} \\
& \begin{array}{lllllll}
\mathrm{H} & 19 & \mathrm{r} 42 & 17 & \mathrm{a} 42 & 16 & \mathrm{~d} 42
\end{array} \\
& \begin{array}{lllllll}
\mathrm{H} & 20 & \mathrm{r} 43 & 13 & \mathrm{a} 43 & 11 & \mathrm{~d} 43
\end{array} \\
& \begin{array}{lllllll}
\mathrm{H} & 22 & \mathrm{r} 44 & 14 & \mathrm{a} 44 & 10 & \mathrm{~d} 44
\end{array} \\
& \begin{array}{lllllll}
\mathrm{H} & 22 & \mathrm{r} 45 & 14 & \mathrm{a} 45 & 10 & \mathrm{~d} 45
\end{array} \\
& \begin{array}{lllllll}
\mathrm{H} & 22 & \mathrm{r} 46 & 14 & \mathrm{a} 46 & 10 & \mathrm{~d} 46
\end{array} \\
& \begin{array}{lllllll}
\mathrm{H} & 24 & \mathrm{r} 47 & 20 & \mathrm{a} 47 & 13 & \mathrm{~d} 47
\end{array} \\
& \begin{array}{lllllll}
\mathrm{H} & 24 & \mathrm{r} 48 & 20 & \mathrm{a} 48 & 13 & \mathrm{~d} 48
\end{array} \\
& \begin{array}{lllllll}
\mathrm{H} & 24 & \mathrm{r} 49 & 20 & \mathrm{a} 49 & 13 & \mathrm{~d} 49
\end{array} \\
& \text { Variables: } \\
& \mathrm{r} 2=1.3996 \\
& \text { r3 }=1.4063 \\
& \mathrm{a} 3=121.01 \\
& \mathrm{r} 4=1.3916 \\
& \mathrm{a} 4=120.24 \\
& \mathrm{~d} 4=359.11 \\
& \mathrm{r} 5=1.4054 \\
& \mathrm{a} 5=119.36 \\
& \mathrm{~d} 5=0.77 \\
& \text { r6 }=1.4003 \\
& \mathrm{a} 6=119.98 \\
& \mathrm{~d} 6=359.88 \\
& \mathrm{r} 7=1.5192 \\
& \mathrm{a} 7=109.25 \\
& \mathrm{~d} 7=180.69 \\
& \mathrm{r} 8=1.5697 \\
& \mathrm{a} 8=102.55 \\
& \mathrm{~d} 8=352.45 \\
& \mathrm{r} 9=1.3958
\end{aligned}
$$




$$
\begin{aligned}
& \mathrm{a} 9=127.95 \\
& \mathrm{~d} 9=181.04 \\
& \mathrm{r} 10=1.4747 \\
& \mathrm{a} 10=105.33 \\
& \mathrm{~d} 10=133.62 \\
& \mathrm{r} 11=1.4772 \\
& \mathrm{a} 11=113.27 \\
& \mathrm{~d} 11=357.75 \\
& \mathrm{r} 12=1.5299 \\
& \mathrm{a} 12=103.97 \\
& \mathrm{~d} 12=345.73 \\
& \mathrm{r} 13=1.5563 \\
& \mathrm{a} 13=112.86 \\
& \mathrm{~d} 13=108.96 \\
& \mathrm{r} 14=1.3622 \\
& \mathrm{a} 14=125.26 \\
& \mathrm{~d} 14=186.64 \\
& \mathrm{r} 15=1.5523 \\
& \mathrm{a} 15=113.68 \\
& \mathrm{~d} 15=229.71 \\
& \mathrm{r} 16=1.5031 \\
& \mathrm{a} 16=114.93 \\
& \mathrm{~d} 16=59.89 \\
& \mathrm{r} 17=1.3482 \\
& \mathrm{a} 17=127.87 \\
& \mathrm{~d} 17=122.33 \\
& \mathrm{r} 18=1.5099 \\
& \mathrm{a} 18=120.79 \\
& \mathrm{~d} 18=178.97 \\
& \mathrm{r} 19=1.5091 \\
& \mathrm{a} 19=124.81 \\
& \mathrm{a} 19.59
\end{aligned}
$$




$$
\begin{aligned}
& \mathrm{d} 21=247.98 \\
& \mathrm{r} 22=1.5145 \\
& \mathrm{a} 22=118.24 \\
& \mathrm{~d} 22=354.68 \\
& \mathrm{r} 23=1.2363 \\
& \mathrm{a} 23=121.36 \\
& \mathrm{~d} 23=174.04 \\
& \text { r24= } 1.4485 \\
& \mathrm{a} 24=123.52 \\
& \mathrm{~d} 24=181.76 \\
& \mathrm{r} 25=1.1013 \\
& \text { a25 }=108.11 \\
& \mathrm{~d} 25=226.88 \\
& \text { r26 }=1.1020 \\
& \text { a26 }=111.76 \\
& \mathrm{~d} 26=252.01 \\
& \text { r27 }=1.0927 \\
& \mathrm{a} 27=120.87 \\
& \mathrm{~d} 27=180.45 \\
& \text { r28 }=1.0937 \\
& \mathrm{a} 28=120.59 \\
& \mathrm{~d} 28=180.12 \\
& \text { r29= } 1.0926 \\
& \mathrm{a} 29=119.97 \\
& \mathrm{~d} 29=179.53 \\
& \text { r30 }=1.0935 \\
& \mathrm{a} 30=119.68 \\
& \mathrm{~d} 30=179.56 \\
& \text { r31 }=1.0147 \\
& \text { a31 }=116.88 \\
& \mathrm{~d} 31=324.71 \\
& \text { r32 }=1.0987 \\
& \text { a32 }=109.07 \\
& \mathrm{~d} 32=266.76 \\
& \text { r33 }=1.0971 \\
& \mathrm{a} 33=111.26 \\
& \mathrm{~d} 33=147.22
\end{aligned}
$$




$$
\begin{aligned}
& \text { r34 }=1.0972 \\
& \text { a34= } 108.51 \\
& \mathrm{~d} 34=186.06 \\
& \text { r35 }=1.1059 \\
& \text { a } 35=106.25 \\
& \mathrm{~d} 35=299.05 \\
& \text { r36 }=1.0973 \\
& \text { a36 }=115.11 \\
& \mathrm{~d} 36=301.65 \\
& \text { r37 }=1.1049 \\
& \text { a37 }=110.92 \\
& \mathrm{~d} 37=239.48 \\
& \text { r38 }=1.1002 \\
& \text { a38 }=112.13 \\
& \mathrm{~d} 38=0.56 \\
& \text { r39 }=1.1048 \\
& \mathrm{a} 39=110.98 \\
& \mathrm{~d} 39=121.77 \\
& \mathrm{r} 40=1.1046 \\
& \mathrm{a} 40=110.44 \\
& \mathrm{~d} 40=119.24 \\
& \mathrm{r} 41=1.1043 \\
& \mathrm{a} 41=110.45 \\
& \mathrm{~d} 41=236.27 \\
& \mathrm{r} 42=1.0978 \\
& \mathrm{a} 42=113.58 \\
& \mathrm{~d} 42=357.83 \\
& \mathrm{r} 43=1.0234 \\
& \mathrm{a} 43=116.28 \\
& \mathrm{~d} 43=354.58 \\
& \mathrm{r} 44=1.0997 \\
& \mathrm{a} 44=112.86 \\
& \mathrm{~d} 44=319.59 \\
& \mathrm{r} 45=1.1011 \\
& \text { a } 45=109.72 \\
& \mathrm{~d} 45=80.36 \\
& \mathrm{r} 46=1.0968
\end{aligned}
$$


$\mathrm{a} 46=108.10$

$\mathrm{d} 46=198.37$

$\mathrm{r} 47=1.1033$

$\mathrm{a} 47=111.46$

$\mathrm{d} 47=112.33$

$\mathrm{r} 48=1.1019$

$\mathrm{a} 48=110.86$

$\mathrm{d} 48=232.83$

$\mathrm{r} 49=1.0972$

$\mathrm{a} 49=108.72$

$\mathrm{d} 49=352.54$ 


\section{SSS2}

$\begin{array}{ll}0 & 1\end{array}$

C

C 11 r2

C $2 \begin{array}{llll}\text { r } 3 & 1 & \text { a3 }\end{array}$

$\begin{array}{lllllll}\text { C } & 3 & \text { r } 4 & 2 & \text { a } 4 & 1 & \text { d } 4\end{array}$

$\begin{array}{lllllll}\mathrm{C} & 4 & \mathrm{r} 5 & 3 & \mathrm{a} 5 & 2 & \mathrm{~d} 5\end{array}$

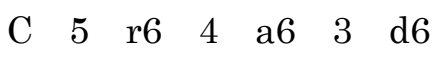

$\begin{array}{lllllll}\mathrm{C} & 3 & \mathrm{r} 7 & 2 & \mathrm{a} 7 & 1 & \mathrm{~d} 7\end{array}$

$\begin{array}{lllllll}\mathrm{C} & 7 & \mathrm{r} 8 & 3 & \mathrm{a} 8 & 2 & \mathrm{~d} 8\end{array}$

$\begin{array}{lllllll}\mathrm{N} & 2 & \mathrm{r} 9 & 1 & \mathrm{a} 9 & 3 & \mathrm{~d} 9\end{array}$

$\begin{array}{lllllll}\mathrm{N} & 8 & \mathrm{r} 10 & 7 & \mathrm{a} 10 & 3 & \mathrm{~d} 10\end{array}$

$\begin{array}{lllllll}\text { C } & 10 & \text { r11 } & 8 & \text { a11 } & 7 & \text { d11 }\end{array}$

$\begin{array}{lllllll}\text { C } & 11 & \text { r12 } & 10 & \text { a } 12 & 8 & \text { d12 }\end{array}$

$\begin{array}{lllllll}\text { C } & 11 & \text { r13 } & 10 & \text { a13 } & 8 & \text { d13 }\end{array}$

$\begin{array}{lllllll}\text { C } & 10 & \text { r14 } & 8 & \text { a14 } & 7 & \text { d14 }\end{array}$

$\begin{array}{lllllll}\mathrm{C} & 7 & \mathrm{r} 15 & 3 & \mathrm{a} 15 & 2 & \mathrm{~d} 15\end{array}$

$\begin{array}{lllllll}\text { C } & 15 & \text { r16 } & 7 & \text { a16 } & 3 & \text { d16 }\end{array}$

$\begin{array}{lllllll}\mathrm{C} & 16 & \mathrm{r} 17 & 15 & \text { a17 } & 7 & \mathrm{~d} 17\end{array}$

$\begin{array}{lllllll}\text { C } & 17 & \text { r18 } & 16 & \text { a18 } & 15 & \text { d18 }\end{array}$

$\begin{array}{lllllll}\mathrm{C} & 17 & \mathrm{r} 19 & 16 & \mathrm{a} 19 & 15 & \mathrm{~d} 19\end{array}$

$\begin{array}{lllllll}\mathrm{N} & 13 & \mathrm{r} 20 & 11 & \mathrm{a} 20 & 10 & \mathrm{~d} 20\end{array}$

$\begin{array}{lllllll}\mathrm{O} & 13 & \mathrm{r} 21 & 11 & \mathrm{a} 21 & 10 & \mathrm{~d} 21\end{array}$

$\begin{array}{lllllll}\text { C } & 14 & \text { r22 } & 10 & \text { a22 } & 8 & \text { d22 }\end{array}$

$\begin{array}{lllllll}\text { O } & 14 & \text { r23 } & 10 & \text { a23 } & 8 & \text { d23 }\end{array}$

$\begin{array}{lllllll}\text { C } & 20 & \text { r24 } & 13 & \text { a24 } & 11 & \text { d24 }\end{array}$

$\begin{array}{lllllll}\mathrm{H} & 11 & \mathrm{r} 25 & 10 & \mathrm{a} 25 & 8 & \mathrm{~d} 25\end{array}$

$\begin{array}{lllllll}\mathrm{H} & 8 & \mathrm{r} 26 & 7 & \mathrm{a} 26 & 3 & \mathrm{~d} 26\end{array}$

$\begin{array}{lllllll}\mathrm{H} & 1 & \mathrm{r} 27 & 2 & \mathrm{a} 27 & 3 & \mathrm{~d} 27\end{array}$

$\begin{array}{lllllll}\mathrm{H} & 4 & \mathrm{r} 28 & 3 & \mathrm{a} 28 & 2 & \mathrm{~d} 28\end{array}$

$\begin{array}{lllllll}\mathrm{H} & 5 & \mathrm{r} 29 & 4 & \mathrm{a} 29 & 3 & \mathrm{~d} 29\end{array}$

$\begin{array}{lllllll}\mathrm{H} & 6 & \mathrm{r} 30 & 5 & \mathrm{a} 30 & 4 & \mathrm{~d} 30\end{array}$

$\begin{array}{lllllll}\mathrm{H} & 9 & \text { r31 } & 2 & \text { a31 } & 1 & \mathrm{~d} 31\end{array}$

$\begin{array}{lllllll}\mathrm{H} & 12 & \text { r32 } & 11 & \text { a32 } & 10 & \text { d } 32\end{array}$

$\begin{array}{lllllll}\mathrm{H} & 12 & \text { r33 } & 11 & \text { a33 } & 10 & \text { d33 }\end{array}$

$\begin{array}{lllllll}\mathrm{H} & 15 & \mathrm{r} 34 & 7 & \mathrm{a} 34 & 3 & \mathrm{~d} 34\end{array}$

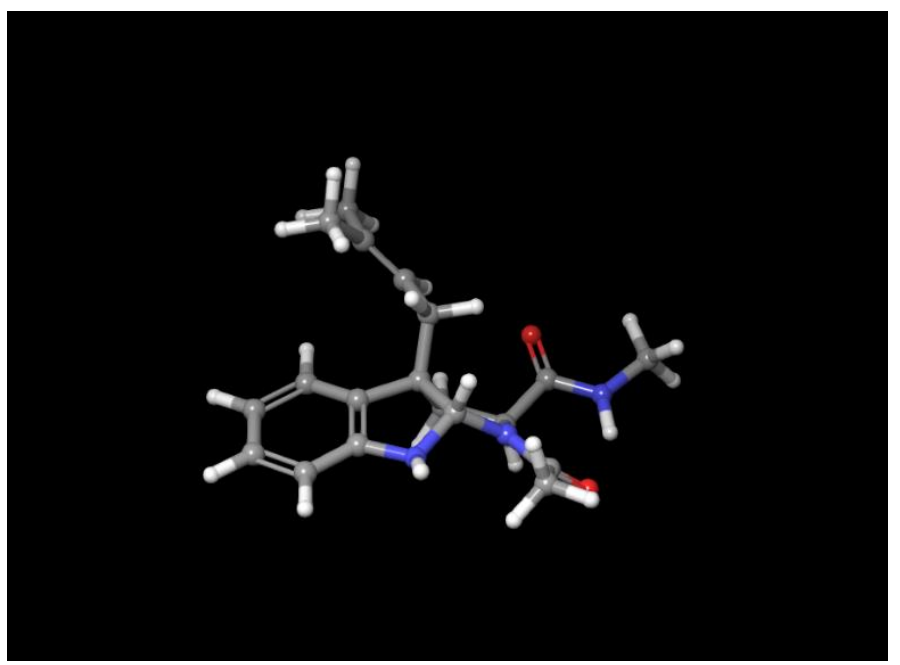




$\begin{array}{lllllll}\mathrm{H} & 15 & \mathrm{r} 35 & 7 & \mathrm{a} 35 & 3 & \mathrm{~d} 35 \\ \mathrm{H} & 16 & \mathrm{r} 36 & 15 & \mathrm{a} 36 & 7 & \mathrm{~d} 36 \\ \mathrm{H} & 18 & \mathrm{r} 37 & 17 & \mathrm{a} 37 & 16 & \mathrm{~d} 37 \\ \mathrm{H} & 18 & \mathrm{r} 38 & 17 & \mathrm{a} 38 & 16 & \mathrm{~d} 38 \\ \mathrm{H} & 18 & \mathrm{r} 39 & 17 & \mathrm{a} 39 & 16 & \mathrm{~d} 39 \\ \mathrm{H} & 19 & \mathrm{r} 40 & 17 & \mathrm{a} 40 & 16 & \mathrm{~d} 40 \\ \mathrm{H} & 19 & \mathrm{r} 41 & 17 & \mathrm{a} 41 & 16 & \mathrm{~d} 41 \\ \mathrm{H} & 19 & \mathrm{r} 42 & 17 & \mathrm{a} 42 & 16 & \mathrm{~d} 42 \\ \mathrm{H} & 20 & \mathrm{r} 43 & 13 & \mathrm{a} 43 & 11 & \mathrm{~d} 43 \\ \mathrm{H} & 22 & \mathrm{r} 44 & 14 & \mathrm{a} 44 & 10 & \mathrm{~d} 44 \\ \mathrm{H} & 22 & \mathrm{r} 45 & 14 & \mathrm{a} 45 & 10 & \mathrm{~d} 45 \\ \mathrm{H} & 22 & \mathrm{r} 46 & 14 & \mathrm{a} 46 & 10 & \mathrm{~d} 46 \\ \mathrm{H} & 24 & \mathrm{r} 47 & 20 & \mathrm{a} 47 & 13 & \mathrm{~d} 47 \\ \mathrm{H} & 24 & \mathrm{r} 48 & 20 & \mathrm{a} 48 & 13 & \mathrm{~d} 48 \\ \mathrm{H} & 24 & \mathrm{r} 49 & 20 & \mathrm{a} 49 & 13 & \mathrm{~d} 49 \\ \text { Variables: } & & & & \end{array}$

$\mathrm{r} 2=1.3992$

$\mathrm{r} 3=1.4085$

a3 $=121.20$

$\mathrm{r} 4=1.3928$

$\mathrm{a} 4=119.91$

$\mathrm{d} 4=358.36$

r5 $=1.4057$

$\mathrm{a} 5=119.44$

$\mathrm{d} 5=1.54$

r6 $=1.3997$

a6 $=120.12$

$\mathrm{d} 6=359.54$

$\mathrm{r} 7=1.5206$

$\mathrm{a} 7=108.90$

$\mathrm{d} 7=180.90$

$\mathrm{r} 8=1.5699$

$\mathrm{a} 8=102.41$

d $8=350.85$

$\mathrm{r} 9=1.3963$

$\mathrm{a} 9=127.61$

$\mathrm{d} 9=181.46$ 


$$
\begin{aligned}
& \mathrm{r} 10=1.4728 \\
& \mathrm{a} 10=105.24 \\
& \mathrm{~d} 10=136.72 \\
& \mathrm{r} 11=1.4787 \\
& \mathrm{a} 11=113.07 \\
& \mathrm{~d} 11=354.72 \\
& \mathrm{r} 12=1.5283 \\
& \mathrm{a} 12=103.66 \\
& \mathrm{~d} 12=346.64 \\
& \mathrm{r} 13=1.5558 \\
& \mathrm{a} 13=113.57 \\
& \mathrm{~d} 13=109.52 \\
& \mathrm{r} 14=1.3623 \\
& \mathrm{a} 14=125.15 \\
& \mathrm{~d} 14=184.37 \\
& \mathrm{r} 15=1.5557 \\
& \mathrm{a} 15=114.63 \\
& \mathrm{~d} 15=231.84 \\
& \mathrm{r} 16=1.5041 \\
& \mathrm{a} 16=116.47 \\
& \mathrm{~d} 16=287.37 \\
& \mathrm{r} 17=1.3479 \\
& \mathrm{a} 17=128.27 \\
& \mathrm{~d} 17=115.88 \\
& \mathrm{r} 18=1.5100 \\
& \mathrm{a} 18=120.64 \\
& \mathrm{~d} 18=177.74 \\
& \mathrm{r} 19=1.5082 \\
& \mathrm{a} 19=125.01 \\
& \mathrm{~d} 19=357.74 \\
& \mathrm{a} 20=1.3493 \\
& \mathrm{~d} 20.32
\end{aligned}
$$




$$
\begin{aligned}
& \mathrm{a} 22=118.21 \\
& \mathrm{~d} 22=355.14 \\
& \mathrm{r} 23=1.2359 \\
& \mathrm{a} 23=121.35 \\
& \mathrm{~d} 23=174.38 \\
& \mathrm{r} 24=1.4483 \\
& \mathrm{a} 24=123.56 \\
& \mathrm{~d} 24=180.94 \\
& \mathrm{r} 25=1.1017 \\
& \mathrm{a} 25=108.17 \\
& \mathrm{~d} 25=227.82 \\
& \mathrm{r} 26=1.1029 \\
& \mathrm{a} 26=111.75 \\
& \mathrm{~d} 26=254.67 \\
& \mathrm{r} 27=1.0927 \\
& \mathrm{a} 27=120.82 \\
& \mathrm{~d} 27=180.52 \\
& \mathrm{r} 28=1.0914 \\
& \mathrm{a} 28=120.30 \\
& \mathrm{~d} 28=180.88 \\
& \mathrm{r} 29=1.0926 \\
& \mathrm{a} 29=119.86 \\
& \mathrm{~d} 29=179.35 \\
& \mathrm{r} 30=1.0935 \\
& \mathrm{a} 30=119.75 \\
& \mathrm{~d} 30=179.64 \\
& \mathrm{r} 31=1.0150 \\
& \mathrm{a} 31=116.76 \\
& \mathrm{~d} 31=325.73 \\
& \mathrm{r} 32=1.0988 \\
& \mathrm{a} 32=108.92 \\
& \mathrm{~d} 32.0959
\end{aligned}
$$




$$
\begin{aligned}
& \mathrm{d} 34=\quad 52.67 \\
& \text { r35 }=1.1034 \\
& \mathrm{a} 35=107.21 \\
& \mathrm{~d} 35=166.96 \\
& \text { r36 }=1.0957 \\
& \text { a36 }=114.44 \\
& \mathrm{~d} 36=292.67 \\
& \text { r37 }=1.1049 \\
& \text { a } 37=110.99 \\
& \mathrm{~d} 37=238.95 \\
& \text { r38 }=1.1002 \\
& \text { a38 }=112.09 \\
& \mathrm{~d} 38=0.09 \\
& \text { r39 }=1.1049 \\
& \text { a39 }=110.95 \\
& \mathrm{~d} 39=121.21 \\
& \mathrm{r} 40=1.1045 \\
& \mathrm{a} 40=110.54 \\
& \mathrm{~d} 40=121.76 \\
& \mathrm{r} 41=1.1045 \\
& \mathrm{a} 41=110.38 \\
& \mathrm{~d} 41=238.93 \\
& \mathrm{r} 42=1.0978 \\
& \mathrm{a} 42=113.58 \\
& \mathrm{~d} 42=0.08 \\
& \mathrm{r} 43=1.0234 \\
& \mathrm{a} 43=116.36 \\
& \mathrm{~d} 43=356.25 \\
& \mathrm{r} 44=1.1014 \\
& \text { a } 44=109.70 \\
& \mathrm{~d} 44=80.33 \\
& \mathrm{r} 45=1.0967 \\
& \mathrm{a} 45=108.12 \\
& \mathrm{~d} 45=198.21 \\
& \mathrm{r} 46=1.0996 \\
& \mathrm{a} 46=112.94 \\
& \mathrm{~d} 46=319.47
\end{aligned}
$$


$\mathrm{r} 47=1.0971$

$\mathrm{a} 47=108.71$

$\mathrm{d} 47=357.20$

$\mathrm{r} 48=1.1030$

$\mathrm{a} 48=111.29$

$\mathrm{d} 48=117.00$

$\mathrm{r} 49=1.1024$

$\mathrm{a} 49=111.09$

$\mathrm{d} 49=237.51$ 


\section{SSS4}
$\begin{array}{ll}0 & 1\end{array}$
C
C $\quad 1 \quad$ r2
C $2 \begin{array}{llll}\text { r } 3 & 1 & \text { a3 }\end{array}$
$\begin{array}{lllllll}\text { C } & 3 & \text { r4 } & 2 & \text { a } 4 & 1 & \mathrm{~d} 4\end{array}$
$\begin{array}{lllllll}\mathrm{C} & 4 & \mathrm{r} 5 & 3 & \mathrm{a} 5 & 2 & \mathrm{~d} 5\end{array}$
C $\quad 5 \quad$ r6 $6 \begin{array}{llll}4 & \text { a6 } & 3 & \text { d6 }\end{array}$
$\begin{array}{lllllll}\mathrm{C} & 3 & \mathrm{r} 7 & 2 & \mathrm{a} 7 & 1 & \mathrm{~d} 7\end{array}$
$\begin{array}{lllllll}\mathrm{C} & 7 & \mathrm{r} 8 & 3 & \mathrm{a} 8 & 2 & \mathrm{~d} 8\end{array}$
$\begin{array}{lllllll}\mathrm{N} & 2 & \mathrm{r} 9 & 1 & \mathrm{a} 9 & 3 & \mathrm{~d} 9\end{array}$
$\begin{array}{lllllll}\mathrm{N} & 8 & \mathrm{r} 10 & 7 & \mathrm{a} 10 & 3 & \mathrm{~d} 10\end{array}$
$\begin{array}{lllllll}\text { C } & 10 & \text { r11 } & 8 & \text { a11 } & 7 & \text { d11 }\end{array}$
$\begin{array}{lllllll}\text { C } & 11 & \text { r12 } & 10 & \text { a12 } & 8 & \text { d12 }\end{array}$
$\begin{array}{lllllll}\text { C } & 11 & \text { r13 } & 10 & \text { a13 } & 8 & \text { d } 13\end{array}$
$\begin{array}{lllllll}\text { C } & 10 & \text { r14 } & 8 & \text { a14 } & 7 & \text { d14 }\end{array}$
$\begin{array}{lllllll}\text { C } & 7 & \text { r15 } & 3 & \text { a15 } & 2 & \text { d } 15\end{array}$
$\begin{array}{lllllll}\mathrm{C} & 15 & \mathrm{r} 16 & 7 & \mathrm{a} 16 & 3 & \mathrm{~d} 16\end{array}$
$\begin{array}{lllllll}\mathrm{C} & 16 & \mathrm{r} 17 & 15 & \text { a17 } & 7 & \mathrm{~d} 17\end{array}$
$\begin{array}{lllllll}\text { C } & 17 & \text { r18 } & 16 & \text { a18 } & 15 & \text { d18 }\end{array}$
$\begin{array}{lllllll}\mathrm{C} & 17 & \mathrm{r} 19 & 16 & \mathrm{a} 19 & 15 & \mathrm{~d} 19\end{array}$
$\begin{array}{lllllll}\mathrm{N} & 13 & \mathrm{r} 20 & 11 & \mathrm{a} 20 & 10 & \mathrm{~d} 20\end{array}$
$\begin{array}{lllllll}\mathrm{O} & 13 & \mathrm{r} 21 & 11 & \mathrm{a} 21 & 10 & \mathrm{~d} 21\end{array}$
$\begin{array}{lllllll}\mathrm{C} & 14 & \mathrm{r} 22 & 10 & \mathrm{a} 22 & 8 & \mathrm{~d} 22\end{array}$
$\begin{array}{lllllll}\mathrm{O} & 14 & \mathrm{r} 23 & 10 & \text { a23 } & 8 & \mathrm{~d} 23\end{array}$
$\begin{array}{lllllll}\text { C } & 20 & \text { r24 } & 13 & \text { a24 } & 11 & \text { d24 }\end{array}$
$\begin{array}{lllllll}\mathrm{H} & 11 & \mathrm{r} 25 & 10 & \mathrm{a} 25 & 8 & \mathrm{~d} 25\end{array}$
$\begin{array}{lllllll}\mathrm{H} & 8 & \mathrm{r} 26 & 7 & \mathrm{a} 26 & 3 & \mathrm{~d} 26\end{array}$
$\begin{array}{lllllll}\mathrm{H} & 1 & \mathrm{r} 27 & 2 & \mathrm{a} 27 & 3 & \mathrm{~d} 27\end{array}$
$\begin{array}{lllllll}\mathrm{H} & 4 & \mathrm{r} 28 & 3 & \mathrm{a} 28 & 2 & \mathrm{~d} 28\end{array}$
$\begin{array}{lllllll}\mathrm{H} & 5 & \mathrm{r} 29 & 4 & \mathrm{a} 29 & 3 & \mathrm{~d} 29\end{array}$
$\begin{array}{lllllll}\mathrm{H} & 6 & \mathrm{r} 30 & 5 & \mathrm{a} 30 & 4 & \mathrm{~d} 30\end{array}$
$\begin{array}{lllllll}\mathrm{H} & 9 & \text { r31 } & 2 & \text { a31 } & 1 & \mathrm{~d} 31\end{array}$
$\begin{array}{lllllll}\mathrm{H} & 12 & \text { r32 } & 11 & \text { a32 } & 10 & \text { d } 32\end{array}$
$\begin{array}{lllllll}\mathrm{H} & 12 & \text { r33 } & 11 & \text { a33 } & 10 & \text { d } 33\end{array}$
$\begin{array}{lllllll}\mathrm{H} & 15 & \text { r34 } & 7 & \text { a34 } & 3 & \text { d34 }\end{array}$

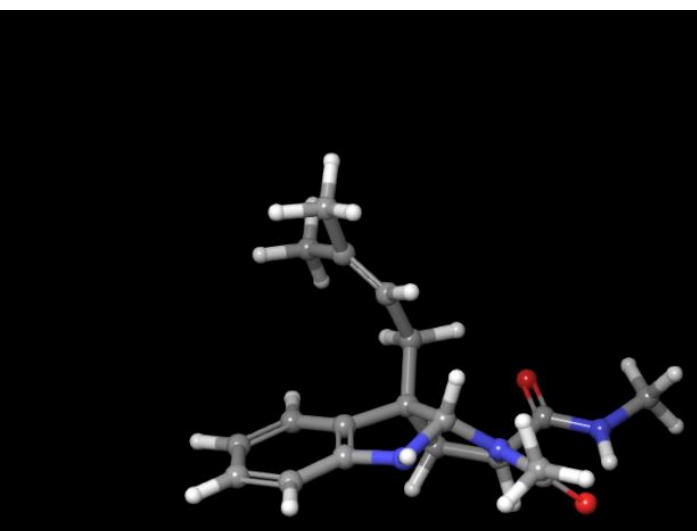




$\begin{array}{lllllll}\mathrm{H} & 15 & \mathrm{r} 35 & 7 & \mathrm{a} 35 & 3 & \mathrm{~d} 35 \\ \mathrm{H} & 16 & \mathrm{r} 36 & 15 & \mathrm{a} 36 & 7 & \mathrm{~d} 36 \\ \mathrm{H} & 18 & \mathrm{r} 37 & 17 & \mathrm{a} 37 & 16 & \mathrm{~d} 37 \\ \mathrm{H} & 18 & \mathrm{r} 38 & 17 & \mathrm{a} 38 & 16 & \mathrm{~d} 38 \\ \mathrm{H} & 18 & \mathrm{r} 39 & 17 & \mathrm{a} 39 & 16 & \mathrm{~d} 39 \\ \mathrm{H} & 19 & \mathrm{r} 40 & 17 & \mathrm{a} 40 & 16 & \mathrm{~d} 40 \\ \mathrm{H} & 19 & \mathrm{r} 41 & 17 & \mathrm{a} 41 & 16 & \mathrm{~d} 41 \\ \mathrm{H} & 19 & \mathrm{r} 42 & 17 & \mathrm{a} 42 & 16 & \mathrm{~d} 42 \\ \mathrm{H} & 20 & \mathrm{r} 43 & 13 & \mathrm{a} 43 & 11 & \mathrm{~d} 43 \\ \mathrm{H} & 22 & \mathrm{r} 44 & 14 & \mathrm{a} 44 & 10 & \mathrm{~d} 44 \\ \mathrm{H} & 22 & \mathrm{r} 45 & 14 & \mathrm{a} 45 & 10 & \mathrm{~d} 45 \\ \mathrm{H} & 22 & \mathrm{r} 46 & 14 & \mathrm{a} 46 & 10 & \mathrm{~d} 46 \\ \mathrm{H} & 24 & \mathrm{r} 47 & 20 & \mathrm{a} 47 & 13 & \mathrm{~d} 47 \\ \mathrm{H} & 24 & \mathrm{r} 48 & 20 & \mathrm{a} 48 & 13 & \mathrm{~d} 48 \\ \mathrm{H} & 24 & \mathrm{r} 49 & 20 & \mathrm{a} 49 & 13 & \mathrm{~d} 49 \\ \text { Variables: } & & & & \end{array}$

$\mathrm{r} 2=1.3994$

$\mathrm{r} 3=1.4060$

$\mathrm{a} 3=121.06$

$\mathrm{r} 4=1.3915$

$\mathrm{a} 4=120.19$

$\mathrm{d} 4=359.03$

r $5=1.4053$

$\mathrm{a} 5=119.39$

$\mathrm{d} 5=0.84$

r6 $=1.4001$

$\mathrm{a} 6=119.99$

$\mathrm{d} 6=359.87$

r7 $=1.5185$

$a 7=109.25$

$\mathrm{d} 7=180.92$

$\mathrm{r} 8=1.5723$

$\mathrm{a} 8=102.55$

$\mathrm{d} 8=352.21$

$\mathrm{r} 9=1.3963$

$\mathrm{a} 9=127.88$

$\mathrm{d} 9=181.10$ 


$$
\begin{aligned}
& \mathrm{r} 10=1.4749 \\
& \mathrm{a} 10=105.32 \\
& \mathrm{~d} 10=133.89 \\
& \mathrm{r} 11=1.4772 \\
& \mathrm{a} 11=113.32 \\
& \mathrm{~d} 11=357.03 \\
& \mathrm{r} 12=1.5289 \\
& \mathrm{a} 12=103.88 \\
& \mathrm{~d} 12=346.11 \\
& \mathrm{r} 13=1.5568 \\
& \mathrm{a} 13=113.28 \\
& \mathrm{~d} 13=109.24 \\
& \mathrm{r} 14=1.3624 \\
& \mathrm{a} 14=125.11 \\
& \mathrm{~d} 14=186.16 \\
& \mathrm{r} 15=1.5523 \\
& \mathrm{a} 15=113.76 \\
& \mathrm{~d} 15=228.88 \\
& \mathrm{r} 16=1.5043 \\
& \mathrm{a} 16=115.52 \\
& \mathrm{~d} 16=57.42 \\
& \mathrm{r} 17=1.3474 \\
& \mathrm{a} 17=128.48 \\
& \mathrm{~d} 17=238.36 \\
& \mathrm{r} 18=1.5101 \\
& \mathrm{a} 18=120.72 \\
& \mathrm{~d} 18=181.12 \\
& \mathrm{r} 19=1.5084 \\
& \mathrm{a} 19=125.02 \\
& \mathrm{~d} 19=
\end{aligned}
$$




$$
\begin{aligned}
& \mathrm{a} 22=118.30 \\
& \mathrm{~d} 22=354.82 \\
& \mathrm{r} 23=1.2361 \\
& \mathrm{a} 23=121.33 \\
& \mathrm{~d} 23=174.17 \\
& \text { r24 }=1.4485 \\
& \text { a24 }=123.50 \\
& \mathrm{~d} 24=181.15 \\
& \text { r25 }=1.1015 \\
& \text { a25 }=108.10 \\
& \mathrm{~d} 25=227.28 \\
& \text { r26 }=1.1012 \\
& \mathrm{a} 26=112.58 \\
& \mathrm{~d} 26=251.95 \\
& \text { r27 }=1.0926 \\
& \mathrm{a} 27=120.88 \\
& \mathrm{~d} 27=180.43 \\
& \text { r28 }=1.0932 \\
& \text { a28 }=120.60 \\
& \mathrm{~d} 28=180.20 \\
& \text { r29 }=1.0926 \\
& \text { a29 }=119.96 \\
& \mathrm{~d} 29=179.52 \\
& \text { r30 }=1.0935 \\
& \text { a30 }=119.69 \\
& \mathrm{~d} 30=179.57 \\
& \text { r31 }=1.0148 \\
& \text { a31 }=116.91 \\
& \mathrm{~d} 31=324.83 \\
& \text { r32 }=1.0986 \\
& \text { a32 }=109.10 \\
& \mathrm{~d} 32=267.00 \\
& \text { r33 }=1.0969 \\
& \mathrm{a} 33=111.14 \\
& \mathrm{~d} 33=147.48 \\
& \text { r34= } 1.1030 \\
& \text { a34= } 107.54
\end{aligned}
$$




$$
\begin{aligned}
& \mathrm{d} 34=180.41 \\
& \mathrm{r} 35=1.1001 \\
& \mathrm{a} 35=107.20 \\
& \mathrm{~d} 35=293.20 \\
& \mathrm{r} 36=1.0975 \\
& \mathrm{a} 36=115.23 \\
& \mathrm{~d} 36=59.99 \\
& \mathrm{r} 37=1.1048 \\
& \mathrm{a} 37=110.91 \\
& \mathrm{~d} 37=238.48 \\
& \mathrm{r} 38=1.1003 \\
& \mathrm{a} 38=112.16 \\
& \mathrm{~d} 38=359.58 \\
& \mathrm{r} 39=1.1049 \\
& \mathrm{a} 39=111.01 \\
& \mathrm{~d} 39=120.74 \\
& \mathrm{r} 40=1.1043 \\
& \mathrm{a} 40=110.45 \\
& \mathrm{~d} 40=121.26 \\
& \mathrm{~d} 46=116=319.41 \\
& \mathrm{r} 41=1.1045 \\
& \mathrm{a} 41=110.45 \\
& \mathrm{~d} 41=238.41 \\
& \mathrm{r} 42=1.0978 \\
& \mathrm{a} 42=113.65 \\
& \mathrm{~d} 42=359.78 \\
& \mathrm{r} 43=1.0233 \\
& \mathrm{a} 43=116.37 \\
& \mathrm{~d} 43=355.76 \\
& \mathrm{r} 44=1.1011 \\
& \mathrm{~d} 44=109.73 \\
& \mathrm{~d} 45=80.25 \\
& \mathrm{~d}=1098
\end{aligned}
$$


$\mathrm{r} 47=1.1024$

$\mathrm{a} 47=111.03$

$\mathrm{d} 47=236.34$

$\mathrm{r} 48=1.0972$

$\mathrm{a} 48=108.68$

$\mathrm{d} 48=356.03$

$\mathrm{r} 49=1.1030$

$\mathrm{a} 49=111.33$

$\mathrm{d} 49=115.83$ 


\section{SSS8}
$\begin{array}{ll}0 & 1\end{array}$
$\mathrm{C}$
C 11 r2
C $2 \begin{array}{llll}\text { r } 3 & 1 & \text { a3 }\end{array}$
$\begin{array}{lllllll}\text { C } & 3 & \text { r4 } & 2 & \text { a } 4 & 1 & \mathrm{~d} 4\end{array}$
$\begin{array}{lllllll}\mathrm{C} & 4 & \mathrm{r} 5 & 3 & \mathrm{a} 5 & 2 & \mathrm{~d} 5\end{array}$

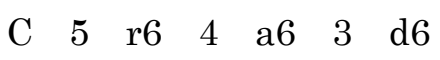
$\begin{array}{lllllll}\mathrm{C} & 3 & \mathrm{r} 7 & 2 & \mathrm{a} 7 & 1 & \mathrm{~d} 7\end{array}$
$\begin{array}{lllllll}\mathrm{C} & 7 & \mathrm{r} 8 & 3 & \mathrm{a} 8 & 2 & \mathrm{~d} 8\end{array}$
$\begin{array}{lllllll}\mathrm{N} & 2 & \mathrm{r} 9 & 1 & \mathrm{a} 9 & 3 & \mathrm{~d} 9\end{array}$
$\begin{array}{lllllll}\mathrm{N} & 8 & \mathrm{r} 10 & 7 & \mathrm{a} 10 & 3 & \mathrm{~d} 10\end{array}$
$\begin{array}{lllllll}\text { C } & 10 & \text { r11 } & 8 & \text { a11 } & 7 & \text { d11 }\end{array}$
$\begin{array}{lllllll}\text { C } & 11 & \text { r12 } & 10 & \text { a12 } & 8 & \text { d12 }\end{array}$
$\begin{array}{lllllll}\text { C } & 11 & \text { r13 } & 10 & \text { a13 } & 8 & \text { d13 }\end{array}$
$\begin{array}{lllllll}\text { C } & 10 & \text { r14 } & 8 & \text { a14 } & 7 & \text { d14 }\end{array}$
$\begin{array}{lllllll}\text { C } & 7 & \text { r15 } & 3 & \text { a15 } & 2 & \text { d } 15\end{array}$
$\begin{array}{lllllll}\text { C } & 15 & \text { r16 } & 7 & \text { a16 } & 3 & \text { d16 }\end{array}$
$\begin{array}{lllllll}\text { C } & 16 & \text { r17 } & 15 & \text { a17 } & 7 & \text { d17 }\end{array}$
$\begin{array}{lllllll}\text { C } & 17 & \text { r18 } & 16 & \text { a18 } & 15 & \text { d18 }\end{array}$
$\begin{array}{lllllll}\mathrm{C} & 17 & \mathrm{r} 19 & 16 & \mathrm{a} 19 & 15 & \mathrm{~d} 19\end{array}$
$\begin{array}{lllllll}\mathrm{N} & 13 & \mathrm{r} 20 & 11 & \mathrm{a} 20 & 10 & \mathrm{~d} 20\end{array}$
$\begin{array}{lllllll}\mathrm{O} & 13 & \mathrm{r} 21 & 11 & \mathrm{a} 21 & 10 & \mathrm{~d} 21\end{array}$
$\begin{array}{lllllll}\text { C } & 14 & \text { r22 } & 10 & \text { a22 } & 8 & \text { d22 }\end{array}$
$\begin{array}{lllllll}\mathrm{O} & 14 & \mathrm{r} 23 & 10 & \text { a23 } & 8 & \mathrm{~d} 23\end{array}$
$\begin{array}{lllllll}\text { C } & 20 & \text { r24 } & 13 & \text { a24 } & 11 & \text { d24 }\end{array}$
$\begin{array}{lllllll}\mathrm{H} & 11 & \mathrm{r} 25 & 10 & \mathrm{a} 25 & 8 & \mathrm{~d} 25\end{array}$
$\begin{array}{lllllll}\mathrm{H} & 8 & \mathrm{r} 26 & 7 & \mathrm{a} 26 & 3 & \mathrm{~d} 26\end{array}$
$\begin{array}{lllllll}\mathrm{H} & 1 & \mathrm{r} 27 & 2 & \mathrm{a} 27 & 3 & \mathrm{~d} 27\end{array}$
$\begin{array}{lllllll}\mathrm{H} & 4 & \mathrm{r} 28 & 3 & \mathrm{a} 28 & 2 & \mathrm{~d} 28\end{array}$
$\begin{array}{lllllll}\mathrm{H} & 5 & \mathrm{r} 29 & 4 & \mathrm{a} 29 & 3 & \mathrm{~d} 29\end{array}$
$\begin{array}{lllllll}\mathrm{H} & 6 & \mathrm{r} 30 & 5 & \mathrm{a} 30 & 4 & \mathrm{~d} 30\end{array}$
$\begin{array}{lllllll}\mathrm{H} & 9 & \text { r31 } & 2 & \text { a31 } & 1 & \mathrm{~d} 31\end{array}$
$\begin{array}{lllllll}\mathrm{H} & 12 & \text { r32 } & 11 & \text { a32 } & 10 & \text { d } 32\end{array}$
$\begin{array}{lllllll}\mathrm{H} & 12 & \text { r33 } & 11 & \text { a33 } & 10 & \text { d33 }\end{array}$
$\begin{array}{lllllll}\mathrm{H} & 15 & \text { r34 } & 7 & \text { a34 } & 3 & \text { d34 }\end{array}$

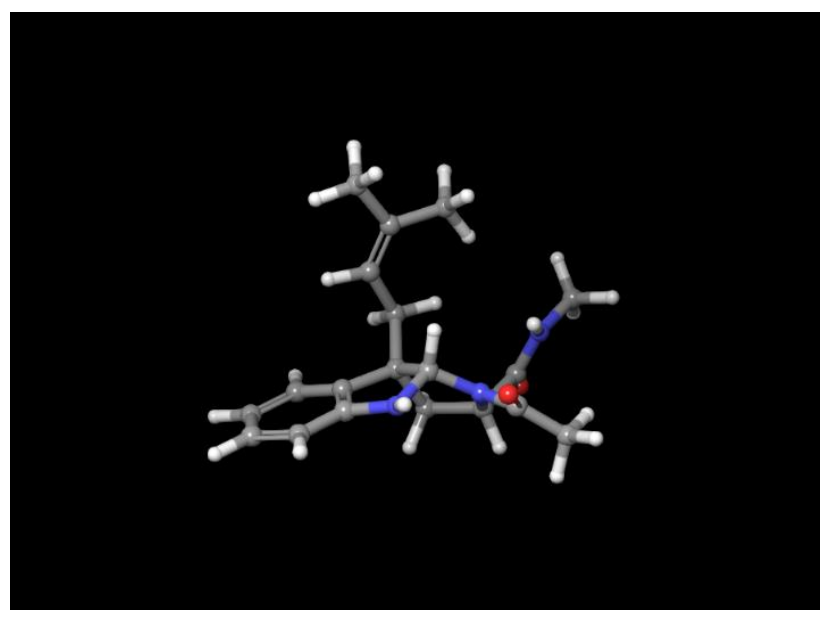




$\begin{array}{lllllll}\mathrm{H} & 15 & \mathrm{r} 35 & 7 & \mathrm{a} 35 & 3 & \mathrm{~d} 35 \\ \mathrm{H} & 16 & \mathrm{r} 36 & 15 & \mathrm{a} 36 & 7 & \mathrm{~d} 36 \\ \mathrm{H} & 18 & \mathrm{r} 37 & 17 & \mathrm{a} 37 & 16 & \mathrm{~d} 37 \\ \mathrm{H} & 18 & \mathrm{r} 38 & 17 & \mathrm{a} 38 & 16 & \mathrm{~d} 38 \\ \mathrm{H} & 18 & \mathrm{r} 39 & 17 & \mathrm{a} 39 & 16 & \mathrm{~d} 39 \\ \mathrm{H} & 19 & \mathrm{r} 40 & 17 & \mathrm{a} 40 & 16 & \mathrm{~d} 40 \\ \mathrm{H} & 19 & \mathrm{r} 41 & 17 & \mathrm{a} 41 & 16 & \mathrm{~d} 41 \\ \mathrm{H} & 19 & \mathrm{r} 42 & 17 & \mathrm{a} 42 & 16 & \mathrm{~d} 42 \\ \mathrm{H} & 20 & \mathrm{r} 43 & 13 & \mathrm{a} 43 & 11 & \mathrm{~d} 43 \\ \mathrm{H} & 22 & \mathrm{r} 44 & 14 & \mathrm{a} 44 & 10 & \mathrm{~d} 44 \\ \mathrm{H} & 22 & \mathrm{r} 45 & 14 & \mathrm{a} 45 & 10 & \mathrm{~d} 45 \\ \mathrm{H} & 22 & \mathrm{r} 46 & 14 & \mathrm{a} 46 & 10 & \mathrm{~d} 46 \\ \mathrm{H} & 24 & \mathrm{r} 47 & 20 & \mathrm{a} 47 & 13 & \mathrm{~d} 47 \\ \mathrm{H} & 24 & \mathrm{r} 48 & 20 & \mathrm{a} 48 & 13 & \mathrm{~d} 48 \\ \mathrm{H} & 24 & \mathrm{r} 49 & 20 & \mathrm{a} 49 & 13 & \mathrm{~d} 49 \\ \text { Variables: } & & & & \end{array}$

$\mathrm{r} 2=1.4007$

$\mathrm{r} 3=1.4110$

a3 $=120.72$

$\mathrm{r} 4=1.3900$

$\mathrm{a} 4=120.39$

$\mathrm{d} 4=359.13$

r5 $=1.4069$

$\mathrm{a} 5=119.40$

$\mathrm{d} 5=0.78$

r6 $=1.3999$

a6 $=119.86$

$\mathrm{d} 6=359.79$

$\mathrm{r} 7=1.5213$

$a 7=108.78$

$\mathrm{d} 7=181.16$

$\mathrm{r} 8=1.5638$

$\mathrm{a} 8=102.42$

$\mathrm{d} 8=350.03$

$\mathrm{r} 9=1.3858$

$\mathrm{a} 9=128.64$

$\mathrm{d} 9=181.55$ 


$$
\begin{aligned}
& \mathrm{r} 10=1.4714 \\
& \mathrm{a} 10=105.62 \\
& \mathrm{~d} 10=136.38 \\
& \mathrm{r} 11=1.4643 \\
& \mathrm{a} 11=113.26 \\
& \mathrm{~d} 11=355.59 \\
& \mathrm{r} 12=1.5428 \\
& \mathrm{a} 12=103.62 \\
& \mathrm{~d} 12=345.93 \\
& \mathrm{r} 13=1.5448 \\
& \mathrm{a} 13=116.42 \\
& \mathrm{~d} 13=109.13 \\
& \text { r14= } 1.3741 \\
& \text { a14 }=118.62 \\
& \text { d14 }=188.01 \\
& \mathrm{r} 15=1.5508 \\
& \mathrm{a} 15=114.35 \\
& \mathrm{~d} 15=226.76 \\
& \text { r16 }=1.5045 \\
& \mathrm{a} 16=114.79 \\
& \mathrm{~d} 16=55.46 \\
& \text { r17 }=1.3478 \\
& \text { a17 }=128.41 \\
& \mathrm{~d} 17=122.48 \\
& \mathrm{r} 18=1.5100 \\
& \text { a18 }=120.65 \\
& \mathrm{~d} 18=178.98 \\
& \mathrm{r} 19=1.5085 \\
& \mathrm{a} 19=125.04 \\
& \mathrm{~d} 19=359.56 \\
& \mathrm{r} 20=1.3527 \\
& \mathrm{a} 20=117.06 \\
& \mathrm{~d} 20=358.44 \\
& \mathrm{r} 21=1.2266 \\
& \mathrm{a} 21=118.76 \\
& \mathrm{~d} 21=177.96 \\
& \mathrm{r} 22=1.5157
\end{aligned}
$$


$\mathrm{a} 22=117.45$

$\mathrm{d} 22=171.98$

r23 $=1.2275$

a23 $=120.48$

$\mathrm{d} 23=352.56$

r24 $=1.4507$

$\mathrm{a} 24=123.49$

$\mathrm{d} 24=178.08$

r25 $=1.1004$

a25 $=111.11$

$\mathrm{d} 25=228.29$

r26 $=1.1026$

$\mathrm{a} 26=112.89$

$\mathrm{d} 26=255.26$

r27 $=1.0926$

a27 $=120.83$

$\mathrm{d} 27=180.51$

r28 $=1.0937$

a28 $=120.60$

$\mathrm{d} 28=180.19$

r29= 1.0924

$\mathrm{a} 29=120.01$

$\mathrm{d} 29=179.44$

r30 $=1.0937$

$\mathrm{a} 30=119.61$

$\mathrm{d} 30=179.58$

r31 $=1.0135$

a31 $=121.15$

$\mathrm{d} 31=333.61$

r32 $=1.0988$

a32 $=108.92$

$\mathrm{d} 32=269.13$

r33 $=1.0983$

$\mathrm{a} 33=111.61$

$\mathrm{d} 33=150.34$

r34 $=1.0993$

a34 $=108.90$ 


$$
\begin{aligned}
& \mathrm{d} 34=180.65 \\
& \text { r35 }=1.1056 \\
& \text { a35 }=106.72 \\
& \text { d } 35=294.43 \\
& \text { r36 }=1.0965 \\
& \text { a36 }=114.62 \\
& \text { d } 36=301.76 \\
& \text { r37 }=1.1048 \\
& \text { a } 37=110.92 \\
& \mathrm{~d} 37=239.36 \\
& \text { r38 }=1.1001 \\
& \text { a38 }=112.13 \\
& \mathrm{~d} 38=0.47 \\
& \text { r39 }=1.1048 \\
& \text { a39 }=110.94 \\
& \mathrm{~d} 39=121.65 \\
& \mathrm{r} 40=1.1044 \\
& \mathrm{a} 40=110.48 \\
& \mathrm{~d} 40=119.10 \\
& \mathrm{r} 41=1.1042 \\
& \mathrm{a} 41=110.47 \\
& \mathrm{~d} 41=236.26 \\
& \mathrm{r} 42=1.0976 \\
& \mathrm{a} 42=113.59 \\
& \mathrm{~d} 42=357.74 \\
& \mathrm{r} 43=1.0141 \\
& \mathrm{a} 43=117.75 \\
& \mathrm{~d} 43=358.60 \\
& \mathrm{r} 44=1.1023 \\
& \mathrm{a} 44=110.26 \\
& \mathrm{~d} 44=291.36 \\
& \mathrm{r} 45=1.0995 \\
& \mathrm{a} 45=112.28 \\
& \mathrm{~d} 45=51.33 \\
& \mathrm{r} 46=1.0966 \\
& \mathrm{a} 46=108.37 \\
& \mathrm{~d} 46=172.43
\end{aligned}
$$


$\mathrm{r} 47=1.0971$

$\mathrm{a} 47=108.16$

$\mathrm{d} 47=\quad 0.66$

$\mathrm{r} 48=1.1016$

$\mathrm{a} 48=111.17$

$\mathrm{d} 48=120.18$

$\mathrm{r} 49=1.1017$

$\mathrm{a} 49=111.08$

$\mathrm{d} 49=241.06$ 


\section{SSS11}
$\begin{array}{ll}0 & 1\end{array}$
C
C 11 r2
C $2 \begin{array}{llll}\text { r } 3 & 1 & \text { a3 }\end{array}$
$\begin{array}{lllllll}\text { C } & 3 & \text { r4 } & 2 & \text { a } 4 & 1 & \mathrm{~d} 4\end{array}$
$\begin{array}{lllllll}\mathrm{C} & 4 & \mathrm{r} 5 & 3 & \mathrm{a} 5 & 2 & \mathrm{~d} 5\end{array}$
$\begin{array}{lllllll}\mathrm{C} & 5 & \mathrm{r} 6 & 4 & \mathrm{a} 6 & 3 & \mathrm{~d} 6\end{array}$
$\begin{array}{lllllll}\text { C } & 3 & \text { r7 } & 2 & \text { a7 } & 1 & \text { d7 }\end{array}$
$\begin{array}{lllllll}\mathrm{C} & 7 & \mathrm{r} 8 & 3 & \mathrm{a} 8 & 2 & \mathrm{~d} 8\end{array}$
$\begin{array}{lllllll}\mathrm{N} & 2 & \mathrm{r} 9 & 1 & \mathrm{a} 9 & 3 & \mathrm{~d} 9\end{array}$
$\begin{array}{lllllll}\mathrm{N} & 8 & \mathrm{r} 10 & 7 & \mathrm{a} 10 & 3 & \mathrm{~d} 10\end{array}$
$\begin{array}{lllllll}\text { C } & 10 & \text { r11 } & 8 & \text { a11 } & 7 & \text { d11 }\end{array}$
$\begin{array}{lllllll}\text { C } & 11 & \text { r12 } & 10 & \text { a12 } & 8 & \text { d12 }\end{array}$
$\begin{array}{lllllll}\text { C } & 11 & \text { r13 } & 10 & \text { a13 } & 8 & \text { d } 13\end{array}$
$\begin{array}{lllllll}\text { C } & 10 & \text { r14 } & 8 & \text { a14 } & 7 & \text { d14 }\end{array}$
$\begin{array}{lllllll}\text { C } & 7 & \text { r15 } & 3 & \text { a15 } & 2 & \text { d } 15\end{array}$
$\begin{array}{lllllll}\mathrm{C} & 15 & \mathrm{r} 16 & 7 & \mathrm{a} 16 & 3 & \mathrm{~d} 16\end{array}$
$\begin{array}{lllllll}\text { C } & 16 & \text { r17 } & 15 & \text { a17 } & 7 & \text { d17 }\end{array}$
$\begin{array}{lllllll}\text { C } & 17 & \text { r18 } & 16 & \text { a18 } & 15 & \text { d18 }\end{array}$
$\begin{array}{lllllll}\mathrm{C} & 17 & \mathrm{r} 19 & 16 & \mathrm{a} 19 & 15 & \mathrm{~d} 19\end{array}$
$\begin{array}{lllllll}\mathrm{N} & 13 & \mathrm{r} 20 & 11 & \mathrm{a} 20 & 10 & \mathrm{~d} 20\end{array}$
$\begin{array}{lllllll}\mathrm{O} & 13 & \mathrm{r} 21 & 11 & \mathrm{a} 21 & 10 & \mathrm{~d} 21\end{array}$
$\begin{array}{lllllll}\text { C } & 14 & \text { r22 } & 10 & \text { a22 } & 8 & \text { d22 }\end{array}$
$\begin{array}{lllllll}\mathrm{O} & 14 & \mathrm{r} 23 & 10 & \text { a23 } & 8 & \mathrm{~d} 23\end{array}$
$\begin{array}{lllllll}\text { C } & 20 & \text { r24 } & 13 & \text { a24 } & 11 & \text { d24 }\end{array}$
$\begin{array}{lllllll}\mathrm{H} & 11 & \mathrm{r} 25 & 10 & \mathrm{a} 25 & 8 & \mathrm{~d} 25\end{array}$
$\begin{array}{lllllll}\mathrm{H} & 8 & \mathrm{r} 26 & 7 & \mathrm{a} 26 & 3 & \mathrm{~d} 26\end{array}$
$\begin{array}{lllllll}\mathrm{H} & 1 & \mathrm{r} 27 & 2 & \mathrm{a} 27 & 3 & \mathrm{~d} 27\end{array}$
$\begin{array}{lllllll}\mathrm{H} & 4 & \mathrm{r} 28 & 3 & \mathrm{a} 28 & 2 & \mathrm{~d} 28\end{array}$
$\begin{array}{lllllll}\mathrm{H} & 5 & \mathrm{r} 29 & 4 & \mathrm{a} 29 & 3 & \mathrm{~d} 29\end{array}$
$\begin{array}{lllllll}\mathrm{H} & 6 & \text { r30 } & 5 & \text { a330 } & 4 & \text { d } 30\end{array}$
$\begin{array}{lllllll}\mathrm{H} & 9 & \text { r31 } & 2 & \text { a31 } & 1 & \mathrm{~d} 31\end{array}$
$\begin{array}{lllllll}\mathrm{H} & 12 & \text { r32 } & 11 & \text { a32 } & 10 & \text { d } 32\end{array}$
$\begin{array}{lllllll}\mathrm{H} & 12 & \text { r33 } & 11 & \text { a33 } & 10 & \text { d33 }\end{array}$
$\begin{array}{lllllll}\mathrm{H} & 15 & \text { r34 } & 7 & \text { a34 } & 3 & \text { d34 }\end{array}$

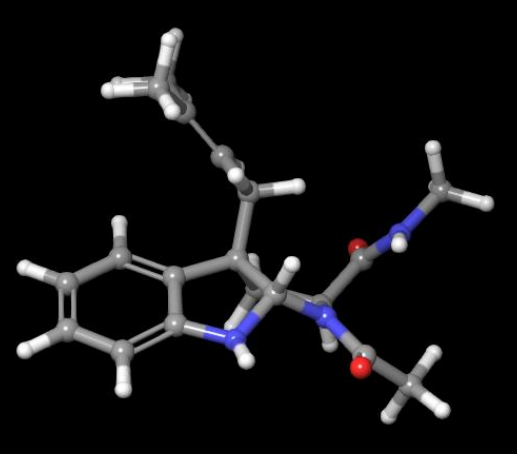



$\begin{array}{lllllll}\mathrm{H} & 15 & \mathrm{r} 35 & 7 & \mathrm{a} 35 & 3 & \mathrm{~d} 35\end{array}$
$\begin{array}{lllllll}\mathrm{H} & 16 & \mathrm{r} 36 & 15 & \text { a36 } & 7 & \mathrm{~d} 36\end{array}$
$\begin{array}{lllllll}\mathrm{H} & 18 & \mathrm{r} 37 & 17 & \mathrm{a} 37 & 16 & \mathrm{~d} 37\end{array}$
$\begin{array}{lllllll}\mathrm{H} & 18 & \mathrm{r} 38 & 17 & \mathrm{a} 38 & 16 & \mathrm{~d} 38\end{array}$
$\begin{array}{lllllll}\mathrm{H} & 18 & \mathrm{r} 39 & 17 & \mathrm{a} 39 & 16 & \mathrm{~d} 39\end{array}$
$\begin{array}{lllllll}\mathrm{H} & 19 & \mathrm{r} 40 & 17 & \mathrm{a} 40 & 16 & \mathrm{~d} 40\end{array}$
$\begin{array}{lllllll}\mathrm{H} & 19 & \mathrm{r} 41 & 17 & \mathrm{a} 41 & 16 & \mathrm{~d} 41\end{array}$
$\begin{array}{lllllll}\mathrm{H} & 19 & \mathrm{r} 42 & 17 & \mathrm{a} 42 & 16 & \mathrm{~d} 42\end{array}$
$\begin{array}{lllllll}\mathrm{H} & 20 & \mathrm{r} 43 & 13 & \mathrm{a} 43 & 11 & \mathrm{~d} 43\end{array}$
$\begin{array}{lllllll}\mathrm{H} & 22 & \mathrm{r} 44 & 14 & \mathrm{a} 44 & 10 & \mathrm{~d} 44\end{array}$
$\begin{array}{lllllll}\mathrm{H} & 22 & \mathrm{r} 45 & 14 & \mathrm{a} 45 & 10 & \mathrm{~d} 45\end{array}$
$\begin{array}{lllllll}\mathrm{H} & 22 & \mathrm{r} 46 & 14 & \mathrm{a} 46 & 10 & \mathrm{~d} 46\end{array}$
$\begin{array}{lllllll}\mathrm{H} & 24 & \mathrm{r} 47 & 20 & \mathrm{a} 47 & 13 & \mathrm{~d} 47\end{array}$
$\begin{array}{lllllll}\mathrm{H} & 24 & \mathrm{r} 48 & 20 & \mathrm{a} 48 & 13 & \mathrm{~d} 48\end{array}$
$\begin{array}{lllllll}\mathrm{H} & 24 & \mathrm{r} 49 & 20 & \mathrm{a} 49 & 13 & \mathrm{~d} 49\end{array}$
Variables:

$$
\begin{aligned}
& \mathrm{r} 2=1.4001 \\
& \mathrm{r} 3=1.4127 \\
& \mathrm{a} 3=120.95 \\
& \mathrm{r} 4=1.3915 \\
& \mathrm{a} 4=120.04 \\
& \mathrm{~d} 4=358.59 \\
& \mathrm{r} 5=1.4069 \\
& \mathrm{a} 5=119.48 \\
& \mathrm{~d} 5= \\
& \mathrm{r} 6=1.3992 \\
& \mathrm{a} 6=120.02 \\
& \mathrm{~d} 6=359.58 \\
& \mathrm{r} 7=1.5226 \\
& \mathrm{a} 7=108.61 \\
& \mathrm{~d} 7=180.99 \\
& \mathrm{r} 8=1.5666 \\
& \mathrm{a} 8=102.19 \\
& \mathrm{~d} 8=349.47 \\
& \mathrm{r} 9=1.3867 \\
& \mathrm{a} 9=128.26 \\
& \mathrm{~d} 9=181.79
\end{aligned}
$$




$$
\begin{aligned}
& \mathrm{r} 10=1.4708 \\
& \mathrm{a} 10=105.61 \\
& \mathrm{~d} 10=137.97 \\
& \mathrm{r} 11=1.4648 \\
& \text { a11 }=112.95 \\
& \mathrm{~d} 11=355.28 \\
& \text { r12 }=1.5406 \\
& \mathrm{a} 12=103.37 \\
& \mathrm{~d} 12=344.74 \\
& \text { r13= } 1.5455 \\
& \mathrm{a} 13=116.37 \\
& \mathrm{~d} 13=107.51 \\
& \mathrm{r} 14=1.3741 \\
& \mathrm{a} 14=118.68 \\
& \mathrm{~d} 14=187.89 \\
& \mathrm{r} 15=1.5540 \\
& \mathrm{a} 15=114.87 \\
& \mathrm{~d} 15=229.67 \\
& \text { r16 }=1.5049 \\
& \text { a16 }=116.46 \\
& \mathrm{~d} 16=287.74 \\
& \text { r17 }=1.3477 \\
& \mathrm{a} 17=128.20 \\
& \mathrm{~d} 17=120.33 \\
& \mathrm{r} 18=1.5097 \\
& \text { a18 }=120.66 \\
& \mathrm{~d} 18=177.85 \\
& \mathrm{r} 19=1.5079 \\
& \mathrm{a} 19=124.99 \\
& \mathrm{~d} 19=357.85 \\
& \mathrm{r} 20=1.3519 \\
& \mathrm{a} 20=117.05 \\
& \mathrm{~d} 20=1.84 \\
& \mathrm{r} 21=1.2270 \\
& \mathrm{a} 21=118.76 \\
& \mathrm{~d} 21=181.11 \\
& \mathrm{r} 22=1.5158
\end{aligned}
$$


$\mathrm{a} 22=117.43$

$\mathrm{d} 22=172.05$

$\mathrm{r} 23=1.2273$

a23 $=120.46$

$\mathrm{d} 23=352.64$

r24 $=1.4504$

$\mathrm{a} 24=123.56$

$\mathrm{d} 24=179.61$

r25 $=1.1003$

$\mathrm{a} 25=111.13$

$\mathrm{d} 25=227.03$

r26 $=1.1038$

$\mathrm{a} 26=112.83$

$\mathrm{d} 26=256.55$

r27 $=1.0926$

a27 $=120.78$

$\mathrm{d} 27=180.57$

r28 $=1.0917$

$\mathrm{a} 28=120.31$

$\mathrm{d} 28=180.74$

r29 $=1.0926$

$\mathrm{a} 29=119.89$

$\mathrm{d} 29=179.36$

r30 $=1.0937$

$\mathrm{a} 30=119.70$

$\mathrm{d} 30=179.64$

r31 $=1.0138$

a31 $=120.85$

$\mathrm{d} 31=333.29$

r32 $=1.0988$

a32 $=108.79$

$\mathrm{d} 32=272.11$

r33 $=1.0969$

a33 $=111.58$

$\mathrm{d} 33=153.67$

r34= 1.0994

a34 $=107.53$ 


$$
\begin{aligned}
& \mathrm{d} 34=52.96 \\
& \mathrm{r} 35=1.1052 \\
& \mathrm{a} 35=107.42 \\
& \mathrm{~d} 35=166.44 \\
& \mathrm{r} 36=1.0968 \\
& \mathrm{a} 36=115.18 \\
& \mathrm{~d} 36=297.64 \\
& \mathrm{r} 37=1.1048 \\
& \mathrm{a} 37=110.93 \\
& \mathrm{~d} 37=238.88 \\
& \mathrm{r} 38=1.1002 \\
& \mathrm{a} 38=112.17 \\
& \mathrm{~d} 38= \\
& \mathrm{r} 39=1.1048 \\
& \mathrm{a} 39=110.91 \\
& \mathrm{~d} 39=121.17 \\
& \mathrm{r} 40=1.1045 \\
& \mathrm{a} 40=110.49 \\
& \mathrm{~d} 46=109 \\
& \mathrm{~d} 40=120.90 \\
& \mathrm{r} 41=1.1044 \\
& \mathrm{a} 41=110.33 \\
& \mathrm{~d} 41=238.01 \\
& \mathrm{r} 42=1.0978 \\
& \mathrm{a} 42=113.67 \\
& \mathrm{~d} 42=359.29 \\
& \mathrm{r} 43=1.0140 \\
& \mathrm{a} 43=117.76 \\
& \mathrm{~d} 43=356.65 \\
& \mathrm{r} 44=1.1022 \\
& \mathrm{a} 44=110.16 \\
& \mathrm{~d} 44=290.39 \\
& \mathrm{~d} 4=1.0995
\end{aligned}
$$


$\mathrm{r} 47=1.1013$

$\mathrm{a} 47=110.92$

$\mathrm{d} 47=236.05$

$\mathrm{r} 48=1.0970$

$\mathrm{a} 48=108.21$

$\mathrm{d} 48=355.67$

$\mathrm{r} 49=1.1021$

$\mathrm{a} 49=111.23$

$\mathrm{d} 49=115.20$ 


\section{SSS12}
$\begin{array}{ll}0 & 1\end{array}$
C
C 11 r2
C $2 \begin{array}{llll}\text { r } 3 & 1 & \text { a3 }\end{array}$
$\begin{array}{lllllll}\text { C } & 3 & \text { r4 } & 2 & \text { a } 4 & 1 & \mathrm{~d} 4\end{array}$
$\begin{array}{lllllll}\mathrm{C} & 4 & \mathrm{r} 5 & 3 & \mathrm{a} 5 & 2 & \mathrm{~d} 5\end{array}$
C $\quad 5 \quad$ r6 $64 \begin{array}{llll}\text { a6 } & 3 & \text { d6 }\end{array}$
$\begin{array}{lllllll}\mathrm{C} & 3 & \mathrm{r} 7 & 2 & \mathrm{a} 7 & 1 & \mathrm{~d} 7\end{array}$
$\begin{array}{lllllll}\mathrm{C} & 7 & \mathrm{r} 8 & 3 & \mathrm{a} 8 & 2 & \mathrm{~d} 8\end{array}$
$\begin{array}{lllllll}\mathrm{N} & 2 & \mathrm{r} 9 & 1 & \mathrm{a} 9 & 3 & \mathrm{~d} 9\end{array}$
$\begin{array}{lllllll}\mathrm{N} & 8 & \mathrm{r} 10 & 7 & \mathrm{a} 10 & 3 & \mathrm{~d} 10\end{array}$
$\begin{array}{lllllll}\text { C } & 10 & \text { r11 } & 8 & \text { a11 } & 7 & \text { d11 }\end{array}$
$\begin{array}{lllllll}\text { C } & 11 & \text { r12 } & 10 & \text { a12 } & 8 & \text { d12 }\end{array}$
$\begin{array}{lllllll}\text { C } & 11 & \text { r13 } & 10 & \text { a13 } & 8 & \text { d } 13\end{array}$
$\begin{array}{lllllll}\text { C } & 10 & \text { r14 } & 8 & \text { a14 } & 7 & \text { d14 }\end{array}$
$\begin{array}{lllllll}\text { C } & 7 & \text { r15 } & 3 & \text { a15 } & 2 & \text { d15 }\end{array}$
$\begin{array}{lllllll}\text { C } & 15 & \text { r16 } & 7 & \text { a16 } & 3 & \text { d } 16\end{array}$
$\begin{array}{lllllll}\mathrm{C} & 16 & \mathrm{r} 17 & 15 & \text { a17 } & 7 & \mathrm{~d} 17\end{array}$
$\begin{array}{lllllll}\text { C } & 17 & \text { r18 } & 16 & \text { a18 } & 15 & \text { d18 }\end{array}$
$\begin{array}{lllllll}\text { C } & 17 & \text { r19 } & 16 & \text { a19 } & 15 & \text { d19 }\end{array}$
$\begin{array}{lllllll}\mathrm{N} & 13 & \mathrm{r} 20 & 11 & \mathrm{a} 20 & 10 & \mathrm{~d} 20\end{array}$
$\begin{array}{lllllll}\mathrm{O} & 13 & \mathrm{r} 21 & 11 & \mathrm{a} 21 & 10 & \mathrm{~d} 21\end{array}$
$\begin{array}{lllllll}\mathrm{C} & 14 & \mathrm{r} 22 & 10 & \mathrm{a} 22 & 8 & \mathrm{~d} 22\end{array}$
$\begin{array}{lllllll}\text { O } & 14 & \text { r23 } & 10 & \text { a23 } & 8 & \text { d23 }\end{array}$
$\begin{array}{lllllll}\text { C } & 20 & \text { r24 } & 13 & \text { a24 } & 11 & \text { d24 }\end{array}$
$\begin{array}{lllllll}\mathrm{H} & 11 & \mathrm{r} 25 & 10 & \mathrm{a} 25 & 8 & \mathrm{~d} 25\end{array}$
$\begin{array}{lllllll}\mathrm{H} & 8 & \mathrm{r} 26 & 7 & \mathrm{a} 26 & 3 & \mathrm{~d} 26\end{array}$
$\begin{array}{lllllll}\mathrm{H} & 1 & \mathrm{r} 27 & 2 & \mathrm{a} 27 & 3 & \mathrm{~d} 27\end{array}$
$\begin{array}{lllllll}\mathrm{H} & 4 & \mathrm{r} 28 & 3 & \mathrm{a} 28 & 2 & \mathrm{~d} 28\end{array}$
$\begin{array}{lllllll}\mathrm{H} & 5 & \mathrm{r} 29 & 4 & \mathrm{a} 29 & 3 & \mathrm{~d} 29\end{array}$
$\begin{array}{lllllll}\mathrm{H} & 6 & \mathrm{r} 30 & 5 & \text { a330 } & 4 & \mathrm{~d} 30\end{array}$
$\begin{array}{lllllll}\mathrm{H} & 9 & \text { r31 } & 2 & \text { a31 } & 1 & \text { d } 31\end{array}$
$\begin{array}{lllllll}\mathrm{H} & 12 & \text { r32 } & 11 & \text { a32 } & 10 & \text { d } 32\end{array}$
$\begin{array}{lllllll}\mathrm{H} & 12 & \text { r33 } & 11 & \text { a33 } & 10 & \text { d } 33\end{array}$
$\begin{array}{lllllll}\mathrm{H} & 15 & \text { r34 } & 7 & \text { a34 } & 3 & \text { d34 }\end{array}$ 


$\begin{array}{lllllll}\mathrm{H} & 15 & \mathrm{r} 35 & 7 & \mathrm{a} 35 & 3 & \mathrm{~d} 35 \\ \mathrm{H} & 16 & \mathrm{r} 36 & 15 & \mathrm{a} 36 & 7 & \mathrm{~d} 36 \\ \mathrm{H} & 18 & \mathrm{r} 37 & 17 & \mathrm{a} 37 & 16 & \mathrm{~d} 37 \\ \mathrm{H} & 18 & \mathrm{r} 38 & 17 & \mathrm{a} 38 & 16 & \mathrm{~d} 38 \\ \mathrm{H} & 18 & \mathrm{r} 39 & 17 & \mathrm{a} 39 & 16 & \mathrm{~d} 39 \\ \mathrm{H} & 19 & \mathrm{r} 40 & 17 & \mathrm{a} 40 & 16 & \mathrm{~d} 40 \\ \mathrm{H} & 19 & \mathrm{r} 41 & 17 & \mathrm{a} 41 & 16 & \mathrm{~d} 41 \\ \mathrm{H} & 19 & \mathrm{r} 42 & 17 & \mathrm{a} 42 & 16 & \mathrm{~d} 42 \\ \mathrm{H} & 20 & \mathrm{r} 43 & 13 & \mathrm{a} 43 & 11 & \mathrm{~d} 43 \\ \mathrm{H} & 22 & \mathrm{r} 44 & 14 & \mathrm{a} 44 & 10 & \mathrm{~d} 44 \\ \mathrm{H} & 22 & \mathrm{r} 45 & 14 & \mathrm{a} 45 & 10 & \mathrm{~d} 45 \\ \mathrm{H} & 22 & \mathrm{r} 46 & 14 & \mathrm{a} 46 & 10 & \mathrm{~d} 46 \\ \mathrm{H} & 24 & \mathrm{r} 47 & 20 & \mathrm{a} 47 & 13 & \mathrm{~d} 47 \\ \mathrm{H} & 24 & \mathrm{r} 48 & 20 & \mathrm{a} 48 & 13 & \mathrm{~d} 48 \\ \mathrm{H} & 24 & \mathrm{r} 49 & 20 & \mathrm{a} 49 & 13 & \mathrm{~d} 49 \\ \text { Variables: } & & & & \end{array}$

$\mathrm{r} 2=1.4012$

$\mathrm{r} 3=1.4117$

a3 $=120.66$

$\mathrm{r} 4=1.3900$

$\mathrm{a} 4=120.37$

$\mathrm{d} 4=359.10$

r5 $=1.4068$

$\mathrm{a} 5=119.46$

$\mathrm{d} 5=\quad 0.72$

r6 $=1.3999$

$\mathrm{a} 6=119.83$

$\mathrm{d} 6=359.92$

$\mathrm{r} 7=1.5209$

$\mathrm{a} 7=108.95$

$\mathrm{d} 7=180.45$

$\mathrm{r} 8=1.5624$

$\mathrm{a} 8=102.53$

d8 $=351.91$

$\mathrm{r} 9=1.3853$

$\mathrm{a} 9=128.62$

$\mathrm{d} 9=181.46$ 


$$
\begin{aligned}
& \mathrm{r} 10=1.4635 \\
& \mathrm{a} 10=105.71 \\
& \mathrm{~d} 10=134.42 \\
& \mathrm{r} 11=1.4551 \\
& \mathrm{a} 11=113.91 \\
& \mathrm{~d} 11=358.63 \\
& \mathrm{r} 12=1.5482 \\
& \mathrm{a} 12=103.23 \\
& \mathrm{~d} 12=343.94 \\
& \mathrm{r} 13=1.5469 \\
& \mathrm{a} 13=112.43 \\
& \mathrm{~d} 13=105.73 \\
& \mathrm{r} 14=1.3666 \\
& \mathrm{a} 14=118.94 \\
& \mathrm{~d} 14=174.12 \\
& \mathrm{r} 15=1.5525 \\
& \mathrm{a} 15=114.29 \\
& \mathrm{~d} 15=229.03 \\
& \mathrm{r} 16=1.5046 \\
& \mathrm{a} 16=115.14 \\
& \mathrm{~d} 16=56.08 \\
& \mathrm{r} 17=1.3480 \\
& \mathrm{a} 17=127.68 \\
& \mathrm{~d} 17=112.50 \\
& \mathrm{r} 18=1.5097 \\
& \mathrm{a} 18=120.74 \\
& \mathrm{~d} 18=178.62 \\
& \mathrm{r} 19=1.5096 \\
& \mathrm{a} 19=124.72 \\
& \mathrm{~d} 19=359.06 \\
& \mathrm{r} 20=1.3525 \\
& \mathrm{~d} 20.93
\end{aligned}
$$


$\mathrm{a} 22=117.48$

$\mathrm{d} 22=182.28$

r23= 1.2295

a23 $=120.47$

$\mathrm{d} 23=2.73$

r24 $=1.4507$

$\mathrm{a} 24=123.78$

$\mathrm{d} 24=177.22$

r25 $=1.1017$

$\mathrm{a} 25=110.85$

$\mathrm{d} 25=226.56$

r26 $=1.1012$

$\mathrm{a} 26=112.96$

$\mathrm{d} 26=253.03$

r27 $=1.0927$

$\mathrm{a} 27=120.81$

$\mathrm{d} 27=180.54$

r28= 1.0939

$\mathrm{a} 28=120.53$

$\mathrm{d} 28=180.11$

r29 $=1.0925$

$\mathrm{a} 29=120.03$

$\mathrm{d} 29=179.50$

r30 $=1.0937$

$\mathrm{a} 30=119.61$

$\mathrm{d} 30=179.52$

r31 $=1.0138$

a31 $=120.78$

$\mathrm{d} 31=332.21$

r32 $=1.0990$

$\mathrm{a} 32=108.68$

$\mathrm{d} 32=269.01$

r33 $=1.0995$

a33= 112.34

$\mathrm{d} 33=150.12$

r34 $=1.0980$

a34= 108.33 


$$
\begin{aligned}
& \mathrm{d} 34=180.80 \\
& \mathrm{r} 35=1.1053 \\
& \mathrm{a} 35=106.80 \\
& \mathrm{~d} 35=294.99 \\
& \mathrm{r} 36=1.0967 \\
& \mathrm{a} 36=115.15 \\
& \mathrm{~d} 36=292.09 \\
& \mathrm{r} 37=1.1049 \\
& \mathrm{a} 37=110.88 \\
& \mathrm{~d} 37=238.59 \\
& \mathrm{r} 38=1.1003 \\
& \mathrm{a} 38=112.17 \\
& \mathrm{~d} 38=359.66 \\
& \mathrm{r} 39=1.1047 \\
& \mathrm{a} 39=110.99 \\
& \mathrm{~d} 39=120.90 \\
& \mathrm{r} 40=1.1051 \\
& \mathrm{a} 40=110.51 \\
& \mathrm{~d} 40=115.55 \\
& \mathrm{~d} 46=18=186.09 \\
& \mathrm{r} 41=1.1044 \\
& \mathrm{a} 41=110.12 \\
& \mathrm{~d} 41=232.30 \\
& \mathrm{r} 42=1.0979 \\
& \mathrm{a} 42=113.74 \\
& \mathrm{~d} 42=353.44 \\
& \mathrm{r} 43=1.0144 \\
& \mathrm{a} 43=118.44 \\
& \mathrm{~d} 43=357.09 \\
& \mathrm{r} 44=1.1010 \\
& \mathrm{~d} 44=111.75 \\
& \mathrm{~d} 45=306.52 \\
& \mathrm{~d}=1010
\end{aligned}
$$


$\mathrm{r} 47=1.0969$

$\mathrm{a} 47=108.22$

$\mathrm{d} 47=\quad 1.89$

$\mathrm{r} 48=1.1017$

$\mathrm{a} 48=111.01$

$\mathrm{d} 48=121.39$

$\mathrm{r} 49=1.1021$

$\mathrm{a} 49=111.22$

$\mathrm{d} 49=242.25$ 


\section{SSS15}
$\begin{array}{ll}0 & 1\end{array}$
C
C 11 r2
C $2 \begin{array}{llll}\text { r } 3 & 1 & \text { a3 }\end{array}$
$\begin{array}{lllllll}\mathrm{C} & 3 & \mathrm{r} 4 & 2 & \mathrm{a} 4 & 1 & \mathrm{~d} 4\end{array}$
$\begin{array}{lllllll}\mathrm{C} & 4 & \mathrm{r} 5 & 3 & \mathrm{a} 5 & 2 & \mathrm{~d} 5\end{array}$
C $\quad 5 \quad$ r6 $64 \begin{array}{llll}\text { a6 } & 3 & \text { d6 }\end{array}$
$\begin{array}{lllllll}\mathrm{C} & 3 & \mathrm{r} 7 & 2 & \mathrm{a} 7 & 1 & \mathrm{~d} 7\end{array}$
$\begin{array}{lllllll}\mathrm{C} & 7 & \mathrm{r} 8 & 3 & \mathrm{a} 8 & 2 & \mathrm{~d} 8\end{array}$
$\begin{array}{lllllll}\mathrm{N} & 2 & \mathrm{r} 9 & 1 & \mathrm{a} 9 & 3 & \mathrm{~d} 9\end{array}$
$\begin{array}{lllllll}\mathrm{N} & 8 & \mathrm{r} 10 & 7 & \mathrm{a} 10 & 3 & \mathrm{~d} 10\end{array}$
$\begin{array}{lllllll}\text { C } & 10 & \text { r11 } & 8 & \text { a11 } & 7 & \text { d11 }\end{array}$
$\begin{array}{lllllll}\text { C } & 11 & \text { r12 } & 10 & \text { a12 } & 8 & \text { d12 }\end{array}$
$\begin{array}{lllllll}\text { C } & 11 & \text { r13 } & 10 & \text { a13 } & 8 & \text { d } 13\end{array}$
$\begin{array}{lllllll}\text { C } & 10 & \text { r14 } & 8 & \text { a14 } & 7 & \text { d14 }\end{array}$
$\begin{array}{lllllll}\text { C } & 7 & \text { r15 } & 3 & \text { a15 } & 2 & \text { d15 }\end{array}$
$\begin{array}{lllllll}\text { C } & 15 & \text { r16 } & 7 & \text { a16 } & 3 & \text { d16 }\end{array}$
$\begin{array}{lllllll}\text { C } & 16 & \text { r17 } & 15 & \text { a17 } & 7 & \text { d17 }\end{array}$
$\begin{array}{lllllll}\text { C } & 17 & \text { r18 } & 16 & \text { a18 } & 15 & \text { d18 }\end{array}$
$\begin{array}{lllllll}\mathrm{C} & 17 & \mathrm{r} 19 & 16 & \mathrm{a} 19 & 15 & \mathrm{~d} 19\end{array}$
$\begin{array}{lllllll}\mathrm{N} & 13 & \mathrm{r} 20 & 11 & \mathrm{a} 20 & 10 & \mathrm{~d} 20\end{array}$
$\begin{array}{lllllll}\mathrm{O} & 13 & \mathrm{r} 21 & 11 & \mathrm{a} 21 & 10 & \mathrm{~d} 21\end{array}$
$\begin{array}{lllllll}\text { C } & 14 & \text { r22 } & 10 & \text { a22 } & 8 & \text { d22 }\end{array}$
$\begin{array}{lllllll}\mathrm{O} & 14 & \mathrm{r} 23 & 10 & \text { a23 } & 8 & \mathrm{~d} 23\end{array}$
$\begin{array}{lllllll}\text { C } & 20 & \text { r24 } & 13 & \text { a24 } & 11 & \text { d24 }\end{array}$
$\begin{array}{lllllll}\mathrm{H} & 11 & \mathrm{r} 25 & 10 & \mathrm{a} 25 & 8 & \mathrm{~d} 25\end{array}$
$\begin{array}{lllllll}\mathrm{H} & 8 & \mathrm{r} 26 & 7 & \mathrm{a} 26 & 3 & \mathrm{~d} 26\end{array}$
$\begin{array}{lllllll}\mathrm{H} & 1 & \mathrm{r} 27 & 2 & \mathrm{a} 27 & 3 & \mathrm{~d} 27\end{array}$
$\begin{array}{lllllll}\mathrm{H} & 4 & \mathrm{r} 28 & 3 & \mathrm{a} 28 & 2 & \mathrm{~d} 28\end{array}$
$\begin{array}{lllllll}\mathrm{H} & 5 & \mathrm{r} 29 & 4 & \mathrm{a} 29 & 3 & \mathrm{~d} 29\end{array}$
$\begin{array}{lllllll}\mathrm{H} & 6 & \text { r30 } & 5 & \text { a330 } & 4 & \text { d } 30\end{array}$
$\begin{array}{lllllll}\mathrm{H} & 9 & \text { r31 } & 2 & \text { a31 } & 1 & \mathrm{~d} 31\end{array}$
$\begin{array}{lllllll}\mathrm{H} & 12 & \text { r32 } & 11 & \text { a32 } & 10 & \text { d } 32\end{array}$
$\begin{array}{lllllll}\mathrm{H} & 12 & \text { r33 } & 11 & \text { a33 } & 10 & \text { d33 }\end{array}$
$\begin{array}{lllllll}\mathrm{H} & 15 & \text { r34 } & 7 & \text { a34 } & 3 & \text { d34 }\end{array}$ 


$\begin{array}{lllllll}\mathrm{H} & 15 & \mathrm{r} 35 & 7 & \mathrm{a} 35 & 3 & \mathrm{~d} 35 \\ \mathrm{H} & 16 & \mathrm{r} 36 & 15 & \mathrm{a} 36 & 7 & \mathrm{~d} 36 \\ \mathrm{H} & 18 & \mathrm{r} 37 & 17 & \mathrm{a} 37 & 16 & \mathrm{~d} 37 \\ \mathrm{H} & 18 & \mathrm{r} 38 & 17 & \mathrm{a} 38 & 16 & \mathrm{~d} 38 \\ \mathrm{H} & 18 & \mathrm{r} 39 & 17 & \mathrm{a} 39 & 16 & \mathrm{~d} 39 \\ \mathrm{H} & 19 & \mathrm{r} 40 & 17 & \mathrm{a} 40 & 16 & \mathrm{~d} 40 \\ \mathrm{H} & 19 & \mathrm{r} 41 & 17 & \mathrm{a} 41 & 16 & \mathrm{~d} 41 \\ \mathrm{H} & 19 & \mathrm{r} 42 & 17 & \mathrm{a} 42 & 16 & \mathrm{~d} 42 \\ \mathrm{H} & 20 & \mathrm{r} 43 & 13 & \mathrm{a} 43 & 11 & \mathrm{~d} 43 \\ \mathrm{H} & 22 & \mathrm{r} 44 & 14 & \mathrm{a} 44 & 10 & \mathrm{~d} 44 \\ \mathrm{H} & 22 & \mathrm{r} 45 & 14 & \mathrm{a} 45 & 10 & \mathrm{~d} 45 \\ \mathrm{H} & 22 & \mathrm{r} 46 & 14 & \mathrm{a} 46 & 10 & \mathrm{~d} 46 \\ \mathrm{H} & 24 & \mathrm{r} 47 & 20 & \mathrm{a} 47 & 13 & \mathrm{~d} 47 \\ \mathrm{H} & 24 & \mathrm{r} 48 & 20 & \mathrm{a} 48 & 13 & \mathrm{~d} 48 \\ \mathrm{H} & 24 & \mathrm{r} 49 & 20 & \mathrm{a} 49 & 13 & \mathrm{~d} 49 \\ \text { Variables: } & & & & \end{array}$

Variables:

$\mathrm{r} 2=1.4003$

$\mathrm{r} 3=1.4108$

$\mathrm{a} 3=120.80$

$\mathrm{r} 4=1.3897$

$\mathrm{a} 4=120.32$

$\mathrm{d} 4=359.12$

r $5=1.4067$

$\mathrm{a} 5=119.42$

$\mathrm{d} 5=0.76$

r6 $=1.3996$

a6 $=119.89$

$\mathrm{d} 6=359.83$

$\mathrm{r} 7=1.5203$

$\mathrm{a} 7=108.79$

$\mathrm{d} 7=181.13$

$\mathrm{r} 8=1.5665$

$\mathrm{a} 8=102.37$

$\mathrm{d} 8=349.67$

$\mathrm{r} 9=1.3868$

$\mathrm{a} 9=128.56$

$\mathrm{d} 9=181.47$ 


$$
\begin{aligned}
& \mathrm{r} 10=1.4703 \\
& \mathrm{a} 10=105.61 \\
& \mathrm{~d} 10=136.99 \\
& \mathrm{r} 11=1.4639 \\
& \mathrm{a} 11=113.35 \\
& \mathrm{~d} 11=355.06 \\
& \mathrm{r} 12=1.5427 \\
& \mathrm{a} 12=103.44 \\
& \mathrm{~d} 12=346.00 \\
& \mathrm{r} 13=1.5446 \\
& \mathrm{a} 13=116.30 \\
& \mathrm{~d} 13=108.96 \\
& \mathrm{r} 14=1.3740 \\
& \mathrm{a} 14=118.60 \\
& \mathrm{~d} 14=187.04 \\
& \mathrm{r} 15=1.5509 \\
& \mathrm{a} 15=114.37 \\
& \mathrm{~d} 15=225.59 \\
& \mathrm{r} 16=1.5047 \\
& \mathrm{a} 16=115.46 \\
& \mathrm{~d} 16=55.81 \\
& \mathrm{r} 22=1.5153 \\
& \mathrm{r} 17=1.3471 \\
& \mathrm{a} 17=128.35 \\
& \mathrm{~d} 17=237.46 \\
& \mathrm{r} 18=1.5099 \\
& \mathrm{a} 18=120.68 \\
& \mathrm{~d} 18=180.84 \\
& \mathrm{r} 19=1.5083 \\
& \mathrm{a} 19=125.03 \\
& \mathrm{~d} 19=
\end{aligned}
$$




$$
\begin{aligned}
& \mathrm{a} 22=117.38 \\
& \mathrm{~d} 22=172.59 \\
& \mathrm{r} 23=1.2274 \\
& \mathrm{a} 23=120.44 \\
& \mathrm{~d} 23=353.09 \\
& \mathrm{r} 24=1.4502 \\
& \mathrm{a} 24=123.60 \\
& \mathrm{~d} 24=179.92 \\
& \mathrm{r} 25=1.1002 \\
& \mathrm{a} 25=111.17 \\
& \mathrm{~d} 25=228.31 \\
& \mathrm{r} 26=1.1021 \\
& \mathrm{a} 26=113.75 \\
& \mathrm{~d} 26=255.66 \\
& \mathrm{r} 27=1.0926 \\
& \mathrm{a} 27=120.84 \\
& \mathrm{~d} 27=180.53 \\
& \mathrm{r} 28=1.0933 \\
& \mathrm{a} 28=120.60 \\
& \mathrm{~d} 28=180.15 \\
& \mathrm{r} 29=1.0927 \\
& \mathrm{a} 29=119.99 \\
& \mathrm{~d} 29=179.45 \\
& \mathrm{r} 30=1.0936 \\
& \mathrm{a} 30=119.63 \\
& \mathrm{~d} 30=179.55 \\
& \mathrm{r} 31=1.0136 \\
& \mathrm{a} 31=120.99 \\
& \mathrm{~d} 31=333.55 \\
& \mathrm{r} 32=1.0987 \\
& \mathrm{a} 32=108.88 \\
& \mathrm{~d} 32.6959
\end{aligned}
$$




$$
\begin{aligned}
& \mathrm{d} 34=177.61 \\
& \mathrm{r} 35=1.0998 \\
& \mathrm{a} 35=107.60 \\
& \mathrm{~d} 35=291.20 \\
& \mathrm{r} 36=1.0971 \\
& \mathrm{a} 36=115.12 \\
& \mathrm{~d} 36=58.68 \\
& \mathrm{r} 37=1.1047 \\
& \mathrm{a} 37=110.89 \\
& \mathrm{~d} 37=238.51 \\
& \mathrm{r} 38=1.1003 \\
& \mathrm{a} 38=112.15 \\
& \mathrm{~d} 38=359.60 \\
& \mathrm{r} 39=1.1048 \\
& \mathrm{a} 39=110.99 \\
& \mathrm{~d} 39=120.76 \\
& \mathrm{r} 40=1.1045 \\
& \mathrm{a} 40=110.45 \\
& \mathrm{~d} 40=238.02 \\
& \mathrm{~d} 46=1.0967 \\
& \mathrm{r} 41=1.0979 \\
& \mathrm{a} 41=113.70 \\
& \mathrm{~d} 41=359.39 \\
& \mathrm{r} 42=1.1044 \\
& \mathrm{a} 42=110.42 \\
& \mathrm{~d} 42=120.90 \\
& \mathrm{r} 43=1.0141 \\
& \mathrm{a} 43=117.69 \\
& \mathrm{~d} 43=358.12 \\
& \mathrm{r} 44=1.1021 \\
& \mathrm{a} 44=110.31 \\
& \mathrm{~d} 44=291.96 \\
& \mathrm{~d} 45=1.0995 \\
& \mathrm{~d}=136
\end{aligned}
$$


$\mathrm{r} 47=1.1014$

$\mathrm{a} 47=110.97$

$\mathrm{d} 47=237.03$

$\mathrm{r} 48=1.0971$

$\mathrm{a} 48=108.19$

$\mathrm{d} 48=356.64$

$\mathrm{r} 49=1.1020$

$\mathrm{a} 49=111.22$

$\mathrm{d} 49=116.16$ 
Structure and geometry of the optimized $(8 S, 10 R, 16 R)-2$ conformers with $>1 \%$ Boltzmann population

\section{SRR1}

$\begin{array}{ll}0 & 1\end{array}$

$\mathrm{C}$

C $\quad 1 \quad \mathrm{r} 2$

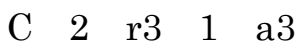

C $3 \begin{array}{llllll}3 & \mathrm{r} 4 & 2 & \mathrm{a} 4 & 1 & \mathrm{~d} 4\end{array}$

C $\quad 4 \quad \begin{array}{rlllll}\text { r5 } & 3 & \text { a5 } & 2 & \text { d5 }\end{array}$

C $5 \begin{array}{llllll}5 & \mathrm{r} 6 & 4 & \mathrm{a} 6 & 3 & \mathrm{~d} 6\end{array}$

$\begin{array}{llllllll}\mathrm{C} & 3 & \mathrm{r} 7 & 2 & \text { a7 } & 1 & \mathrm{~d} 7\end{array}$

$\begin{array}{lllllll}\text { C } & 7 & \text { r8 } & 3 & \text { a8 } & 2 & \mathrm{~d} 8\end{array}$

$\begin{array}{lllllll}\mathrm{N} & 2 & \mathrm{r} 9 & 1 & \mathrm{a} 9 & 3 & \mathrm{~d} 9\end{array}$

$\begin{array}{lllllll}\mathrm{N} & 8 & \mathrm{r} 10 & 7 & \mathrm{a} 10 & 3 & \mathrm{~d} 10\end{array}$

$\begin{array}{lllllll}\mathrm{C} & 10 & \mathrm{r} 11 & 8 & \text { a11 } & 7 & \mathrm{~d} 11\end{array}$

$\begin{array}{lllllll}\mathrm{C} & 11 & \mathrm{r} 12 & 10 & \mathrm{a} 12 & 8 & \mathrm{~d} 12\end{array}$

$\begin{array}{lllllll}\text { C } & 11 & \text { r13 } & 10 & \text { a13 } & 8 & \text { d13 }\end{array}$

$\begin{array}{lllllll}\text { C } & 10 & \text { r14 } & 8 & \text { a14 } & 7 & \text { d14 }\end{array}$

$\begin{array}{lllllll}\text { C } & 7 & \text { r15 } & 3 & \text { a15 } & 2 & \text { d } 15\end{array}$

$\begin{array}{lllllll}\mathrm{C} & 15 & \mathrm{r} 16 & 7 & \mathrm{a} 16 & 3 & \mathrm{~d} 16\end{array}$

$\begin{array}{lllllll}\mathrm{C} & 16 & \mathrm{r} 17 & 15 & \mathrm{a} 17 & 7 & \mathrm{~d} 17\end{array}$

$\begin{array}{lllllll}\text { C } & 17 & \text { r18 } & 16 & \text { a18 } & 15 & \text { d18 }\end{array}$

$\begin{array}{lllllll}\text { C } & 17 & \text { r19 } & 16 & \text { a19 } & 15 & \text { d19 }\end{array}$

$\begin{array}{lllllll}\mathrm{N} & 13 & \mathrm{r} 20 & 11 & \mathrm{a} 20 & 10 & \mathrm{~d} 20\end{array}$

$\begin{array}{lllllll}\mathrm{O} & 13 & \mathrm{r} 21 & 11 & \mathrm{a} 21 & 10 & \mathrm{~d} 21\end{array}$

$\begin{array}{lllllll}\text { C } & 14 & \text { r22 } & 10 & \text { a22 } & 8 & \text { d } 22\end{array}$

$\begin{array}{lllllll}\mathrm{O} & 14 & \mathrm{r} 23 & 10 & \text { a23 } & 8 & \mathrm{~d} 23\end{array}$

$\begin{array}{lllllll}\text { C } & 20 & \text { r24 } & 13 & \text { a24 } & 11 & \text { d24 }\end{array}$

$\begin{array}{lllllll}\mathrm{H} & 11 & \mathrm{r} 25 & 10 & \mathrm{a} 25 & 8 & \mathrm{~d} 25\end{array}$

$\begin{array}{lllllll}\mathrm{H} & 8 & \mathrm{r} 26 & 7 & \mathrm{a} 26 & 3 & \mathrm{~d} 26\end{array}$

$\begin{array}{lllllll}\mathrm{H} & 1 & \mathrm{r} 27 & 2 & \mathrm{a} 27 & 3 & \mathrm{~d} 27\end{array}$

$\begin{array}{lllllll}\mathrm{H} & 4 & \mathrm{r} 28 & 3 & \mathrm{a} 28 & 2 & \mathrm{~d} 28\end{array}$

$\begin{array}{lllllll}\mathrm{H} & 5 & \mathrm{r} 29 & 4 & \mathrm{a} 29 & 3 & \mathrm{~d} 29\end{array}$

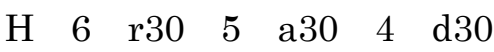

$\begin{array}{lllllll}\mathrm{H} & 9 & \mathrm{r} 31 & 2 & \text { a31 } & 1 & \mathrm{~d} 31\end{array}$ 


$$
\begin{aligned}
& \begin{array}{lllllll}
\mathrm{H} & 12 & \text { r32 } & 11 & \text { a32 } & 10 & \text { d } 32
\end{array} \\
& \begin{array}{lllllll}
\mathrm{H} & 12 & \mathrm{r} 33 & 11 & \mathrm{a} 33 & 10 & \mathrm{~d} 33
\end{array} \\
& \begin{array}{lllllll}
\mathrm{H} & 15 & \mathrm{r} 34 & 7 & \mathrm{a} 34 & 3 & \mathrm{~d} 34
\end{array} \\
& \begin{array}{lllllll}
\mathrm{H} & 15 & \mathrm{r} 35 & 7 & \mathrm{a} 35 & 3 & \mathrm{~d} 35
\end{array} \\
& \begin{array}{lllllll}
\mathrm{H} & 16 & \mathrm{r} 36 & 15 & \mathrm{a} 36 & 7 & \mathrm{~d} 36
\end{array} \\
& \begin{array}{lllllll}
\mathrm{H} & 18 & \mathrm{r} 37 & 17 & \mathrm{a} 37 & 16 & \mathrm{~d} 37
\end{array} \\
& \begin{array}{lllllll}
\mathrm{H} & 18 & \mathrm{r} 38 & 17 & \mathrm{a} 38 & 16 & \mathrm{~d} 38
\end{array} \\
& \begin{array}{lllllll}
\mathrm{H} & 18 & \mathrm{r} 39 & 17 & \mathrm{a} 39 & 16 & \mathrm{~d} 39
\end{array} \\
& \begin{array}{lllllll}
\mathrm{H} & 19 & \mathrm{r} 40 & 17 & \mathrm{a} 40 & 16 & \mathrm{~d} 40
\end{array} \\
& \begin{array}{lllllll}
\mathrm{H} & 19 & \mathrm{r} 41 & 17 & \mathrm{a} 41 & 16 & \mathrm{~d} 41
\end{array} \\
& \begin{array}{lllllll}
\mathrm{H} & 19 & \mathrm{r} 42 & 17 & \mathrm{a} 42 & 16 & \mathrm{~d} 42
\end{array} \\
& \begin{array}{lllllll}
\mathrm{H} & 20 & \mathrm{r} 43 & 13 & \mathrm{a} 43 & 11 & \mathrm{~d} 43
\end{array} \\
& \begin{array}{lllllll}
\mathrm{H} & 22 & \mathrm{r} 44 & 14 & \mathrm{a} 44 & 10 & \mathrm{~d} 44
\end{array} \\
& \begin{array}{lllllll}
\mathrm{H} & 22 & \mathrm{r} 45 & 14 & \mathrm{a} 45 & 10 & \mathrm{~d} 45
\end{array} \\
& \begin{array}{lllllll}
\mathrm{H} & 22 & \mathrm{r} 46 & 14 & \mathrm{a} 46 & 10 & \mathrm{~d} 46
\end{array} \\
& \begin{array}{lllllll}
\mathrm{H} & 24 & \mathrm{r} 47 & 20 & \mathrm{a} 47 & 13 & \mathrm{~d} 47
\end{array} \\
& \begin{array}{lllllll}
\mathrm{H} & 24 & \mathrm{r} 48 & 20 & \mathrm{a} 48 & 13 & \mathrm{~d} 48
\end{array} \\
& \begin{array}{lllllll}
\mathrm{H} & 24 & \mathrm{r} 49 & 20 & \mathrm{a} 49 & 13 & \mathrm{~d} 49
\end{array} \\
& \text { Variables: } \\
& \mathrm{r} 2=1.3999 \\
& \text { r3 }=1.4079 \\
& \text { a3 }=120.96 \\
& \mathrm{r} 4=1.3918 \\
& \mathrm{a} 4=120.17 \\
& \mathrm{~d} 4=\quad 1.18 \\
& \mathrm{r} 5=1.4051 \\
& \mathrm{a} 5=119.46 \\
& \mathrm{~d} 5=359.31 \\
& \mathrm{r} 6=1.4005 \\
& \mathrm{a} 6=119.92 \\
& \mathrm{~d} 6=359.65 \\
& \mathrm{r} 7=1.5234 \\
& \mathrm{a} 7=109.77 \\
& \mathrm{~d} 7=184.49 \\
& \mathrm{r} 8=1.5707 \\
& \mathrm{a} 8=102.22 \\
& \mathrm{~d} 8=353.75
\end{aligned}
$$




$$
\begin{aligned}
& \mathrm{r} 9=1.3923 \\
& \mathrm{a} 9=128.22 \\
& \mathrm{~d} 9=177.33 \\
& \mathrm{r} 10=1.4658 \\
& \mathrm{a} 10=105.06 \\
& \mathrm{~d} 10=243.84 \\
& \mathrm{r} 11=1.4590 \\
& \mathrm{a} 11=112.60 \\
& \mathrm{~d} 11=15.80 \\
& \mathrm{r} 12=1.5431 \\
& \mathrm{a} 12=103.13 \\
& \mathrm{~d} 12=332.09 \\
& \mathrm{r} 13=1.5450 \\
& \mathrm{a} 13=115.93 \\
& \mathrm{~d} 13=95.25 \\
& \mathrm{r} 14=1.3719 \\
& \mathrm{a} 14=119.90 \\
& \mathrm{~d} 14=198.99 \\
& \mathrm{r} 15=1.5602 \\
& \mathrm{a} 15=109.64 \\
& \mathrm{~d} 15=113.43 \\
& \mathrm{r} 16=1.5047 \\
& \mathrm{a} 16=115.79 \\
& \mathrm{~d} 16=188.61 \\
& \mathrm{r} 17=1.3487 \\
& \mathrm{a} 17=127.90 \\
& \mathrm{~d} 17=111.22 \\
& \mathrm{r} 18=1.5096 \\
& \mathrm{a} 18=120.68 \\
& \mathrm{~d} 18=179.30 \\
& \mathrm{r} 19=1.5085 \\
& \mathrm{a} 19.07
\end{aligned}
$$




$$
\begin{aligned}
& \mathrm{a} 21=118.86 \\
& \mathrm{~d} 21=173.88 \\
& \mathrm{r} 22=1.5163 \\
& \mathrm{a} 22=117.12 \\
& \mathrm{~d} 22=177.37 \\
& \mathrm{r} 23=1.2272 \\
& \text { a23 }=120.74 \\
& \mathrm{~d} 23=357.34 \\
& \text { r24= } 1.4494 \\
& \mathrm{a} 24=123.03 \\
& \mathrm{~d} 24=181.81 \\
& \text { r25 }=1.0988 \\
& \mathrm{a} 25=111.03 \\
& \mathrm{~d} 25=214.34 \\
& \text { r26 }=1.0971 \\
& \mathrm{a} 26=114.04 \\
& \mathrm{~d} 26=125.88 \\
& \text { r27 }=1.0926 \\
& \mathrm{a} 27=120.83 \\
& \mathrm{~d} 27=179.70 \\
& \mathrm{r} 28=1.0939 \\
& \mathrm{a} 28=120.53 \\
& \mathrm{~d} 28=179.94 \\
& \text { r29 }=1.0925 \\
& \mathrm{a} 29=120.00 \\
& \mathrm{~d} 29=180.09 \\
& \text { r30 }=1.0935 \\
& \mathrm{a} 30=119.66 \\
& \mathrm{~d} 30=180.53 \\
& \text { r31 }=1.0143 \\
& \text { a31 }=120.29 \\
& \mathrm{~d} 31=33.91 \\
& \text { r32 }=1.0983 \\
& \text { a32 }=108.51 \\
& \mathrm{~d} 32=269.74 \\
& \text { r33 }=1.0981 \\
& \mathrm{a} 33=111.72
\end{aligned}
$$




$$
\begin{aligned}
& \mathrm{d} 33=151.33 \\
& \mathrm{r} 34=1.0997 \\
& \mathrm{a} 34=107.69 \\
& \mathrm{~d} 34=313.31 \\
& \mathrm{r} 35=1.1049 \\
& \mathrm{a} 35=106.79 \\
& \mathrm{~d} 35=66.82 \\
& \mathrm{r} 36=1.0967 \\
& \mathrm{a} 36=115.61 \\
& \mathrm{~d} 36=290.42 \\
& \mathrm{r} 37=1.1046 \\
& \mathrm{a} 37=110.90 \\
& \mathrm{~d} 37=121.74 \\
& \mathrm{r} 38=1.1047 \\
& \mathrm{a} 38=110.88 \\
& \mathrm{~d} 38=239.44 \\
& \mathrm{r} 39=1.1002 \\
& \mathrm{a} 39=112.16 \\
& \mathrm{~d} 39= \\
& \mathrm{d} 45=1.59 \\
& \mathrm{r} 40=1.1044 \\
& \mathrm{a} 40=110.41 \\
& \mathrm{~d} 40=121.28 \\
& \mathrm{r} 41=1.1043 \\
& \mathrm{a} 41=110.37 \\
& \mathrm{~d} 41=238.30 \\
& \mathrm{r} 42=1.0978 \\
& \mathrm{a} 42=113.75 \\
& \mathrm{~d} 42=359.76 \\
& \mathrm{r} 43=1.0183 \\
& \mathrm{a} 43=117.57 \\
& \mathrm{~d} 43=350.42 \\
& \mathrm{~d} 45=1016
\end{aligned}
$$


$\mathrm{r} 46=1.0965$

$\mathrm{a} 46=108.38$

$\mathrm{d} 46=177.24$

$\mathrm{r} 47=1.0973$

$\mathrm{a} 47=108.52$

$\mathrm{d} 47=344.98$

$\mathrm{r} 48=1.1032$

$\mathrm{a} 48=111.53$

$\mathrm{d} 48=104.55$

$\mathrm{r} 49=1.1008$

$\mathrm{a} 49=110.59$

$\mathrm{d} 49=225.26$ 


\section{SRR2}

\section{$\begin{array}{ll}0 & 1\end{array}$}

C

C 11 r2

C $2 \begin{array}{llll}\text { r } 3 & 1 & \text { a3 }\end{array}$

$\begin{array}{lllllll}\text { C } & 3 & \text { r4 } & 2 & \text { a } 4 & 1 & \mathrm{~d} 4\end{array}$

$\begin{array}{lllllll}\mathrm{C} & 4 & \mathrm{r} 5 & 3 & \mathrm{a} 5 & 2 & \mathrm{~d} 5\end{array}$

$\begin{array}{lllllll}\mathrm{C} & 5 & \mathrm{r} 6 & 4 & \mathrm{a} 6 & 3 & \mathrm{~d} 6\end{array}$

$\begin{array}{lllllll}\text { C } & 3 & \text { r7 } & 2 & \text { a7 } & 1 & \text { d7 }\end{array}$

$\begin{array}{lllllll}\mathrm{C} & 7 & \mathrm{r} 8 & 3 & \mathrm{a} 8 & 2 & \mathrm{~d} 8\end{array}$

$\begin{array}{lllllll}\mathrm{N} & 2 & \mathrm{r} 9 & 1 & \mathrm{a} 9 & 3 & \mathrm{~d} 9\end{array}$

$\begin{array}{lllllll}\mathrm{N} & 8 & \mathrm{r} 10 & 7 & \mathrm{a} 10 & 3 & \mathrm{~d} 10\end{array}$

$\begin{array}{lllllll}\text { C } & 10 & \text { r11 } & 8 & \text { a11 } & 7 & \text { d11 }\end{array}$

$\begin{array}{lllllll}\text { C } & 11 & \text { r12 } & 10 & \text { a12 } & 8 & \text { d12 }\end{array}$

$\begin{array}{lllllll}\text { C } & 11 & \text { r13 } & 10 & \text { a13 } & 8 & \text { d13 }\end{array}$

$\begin{array}{lllllll}\text { C } & 10 & \text { r14 } & 8 & \text { a14 } & 7 & \text { d14 }\end{array}$

$\begin{array}{lllllll}\text { C } & 7 & \text { r15 } & 3 & \text { a15 } & 2 & \text { d15 }\end{array}$

$\begin{array}{lllllll}\mathrm{C} & 15 & \mathrm{r} 16 & 7 & \mathrm{a} 16 & 3 & \mathrm{~d} 16\end{array}$

$\begin{array}{lllllll}\text { C } & 16 & \text { r17 } & 15 & \text { a17 } & 7 & \text { d17 }\end{array}$

$\begin{array}{lllllll}\text { C } & 17 & \text { r18 } & 16 & \text { a18 } & 15 & \text { d18 }\end{array}$

$\begin{array}{lllllll}\text { C } & 17 & \text { r19 } & 16 & \text { a19 } & 15 & \text { d19 }\end{array}$

$\begin{array}{lllllll}\mathrm{N} & 13 & \mathrm{r} 20 & 11 & \mathrm{a} 20 & 10 & \mathrm{~d} 20\end{array}$

$\begin{array}{lllllll}\mathrm{O} & 13 & \mathrm{r} 21 & 11 & \mathrm{a} 21 & 10 & \mathrm{~d} 21\end{array}$

$\begin{array}{lllllll}\mathrm{C} & 14 & \mathrm{r} 22 & 10 & \mathrm{a} 22 & 8 & \mathrm{~d} 22\end{array}$

$\begin{array}{lllllll}\mathrm{O} & 14 & \text { r23 } & 10 & \text { a23 } & 8 & \text { d23 }\end{array}$

$\begin{array}{lllllll}\text { C } & 20 & \text { r24 } & 13 & \text { a24 } & 11 & \text { d24 }\end{array}$

$\begin{array}{lllllll}\mathrm{H} & 11 & \mathrm{r} 25 & 10 & \mathrm{a} 25 & 8 & \mathrm{~d} 25\end{array}$

$\begin{array}{lllllll}\mathrm{H} & 8 & \mathrm{r} 26 & 7 & \mathrm{a} 26 & 3 & \mathrm{~d} 26\end{array}$

$\begin{array}{lllllll}\mathrm{H} & 1 & \mathrm{r} 27 & 2 & \mathrm{a} 27 & 3 & \mathrm{~d} 27\end{array}$

$\begin{array}{lllllll}\mathrm{H} & 4 & \mathrm{r} 28 & 3 & \mathrm{a} 28 & 2 & \mathrm{~d} 28\end{array}$

$\begin{array}{lllllll}\mathrm{H} & 5 & \mathrm{r} 29 & 4 & \mathrm{a} 29 & 3 & \mathrm{~d} 29\end{array}$

$\begin{array}{lllllll}\mathrm{H} & 6 & \mathrm{r} 30 & 5 & \text { a330 } & 4 & \mathrm{~d} 30\end{array}$

$\begin{array}{lllllll}\mathrm{H} & 9 & \text { r31 } & 2 & \text { a31 } & 1 & \text { d } 31\end{array}$

$\begin{array}{lllllll}\mathrm{H} & 12 & \text { r32 } & 11 & \text { a32 } & 10 & \text { d32 }\end{array}$

$\begin{array}{lllllll}\mathrm{H} & 12 & \text { r33 } & 11 & \text { a33 } & 10 & \text { d33 }\end{array}$

$\begin{array}{lllllll}\mathrm{H} & 15 & \mathrm{r} 34 & 7 & \mathrm{a} 34 & 3 & \mathrm{~d} 34\end{array}$ 

$\begin{array}{lllllll}\mathrm{H} & 15 & \mathrm{r} 35 & 7 & \mathrm{a} 35 & 3 & \mathrm{~d} 35\end{array}$
$\begin{array}{lllllll}\mathrm{H} & 16 & \mathrm{r} 36 & 15 & \mathrm{a} 36 & 7 & \mathrm{~d} 36\end{array}$
$\begin{array}{lllllll}\mathrm{H} & 18 & \mathrm{r} 37 & 17 & \mathrm{a} 37 & 16 & \mathrm{~d} 37\end{array}$
$\begin{array}{lllllll}\mathrm{H} & 18 & \mathrm{r} 38 & 17 & \mathrm{a} 38 & 16 & \mathrm{~d} 38\end{array}$
$\begin{array}{lllllll}\mathrm{H} & 18 & \mathrm{r} 39 & 17 & \mathrm{a} 39 & 16 & \mathrm{~d} 39\end{array}$
$\begin{array}{lllllll}\mathrm{H} & 19 & \mathrm{r} 40 & 17 & \mathrm{a} 40 & 16 & \mathrm{~d} 40\end{array}$
$\begin{array}{lllllll}\mathrm{H} & 19 & \mathrm{r} 41 & 17 & \mathrm{a} 41 & 16 & \mathrm{~d} 41\end{array}$
$\begin{array}{lllllll}\mathrm{H} & 19 & \mathrm{r} 42 & 17 & \mathrm{a} 42 & 16 & \mathrm{~d} 42\end{array}$
$\begin{array}{lllllll}\mathrm{H} & 20 & \mathrm{r} 43 & 13 & \mathrm{a} 43 & 11 & \mathrm{~d} 43\end{array}$
$\begin{array}{lllllll}\mathrm{H} & 22 & \mathrm{r} 44 & 14 & \mathrm{a} 44 & 10 & \mathrm{~d} 44\end{array}$
$\begin{array}{lllllll}\mathrm{H} & 22 & \mathrm{r} 45 & 14 & \mathrm{a} 45 & 10 & \mathrm{~d} 45\end{array}$
$\begin{array}{lllllll}\mathrm{H} & 22 & \mathrm{r} 46 & 14 & \mathrm{a} 46 & 10 & \mathrm{~d} 46\end{array}$
$\begin{array}{lllllll}\mathrm{H} & 24 & \mathrm{r} 47 & 20 & \mathrm{a} 47 & 13 & \mathrm{~d} 47\end{array}$
$\begin{array}{lllllll}\mathrm{H} & 24 & \mathrm{r} 48 & 20 & \mathrm{a} 48 & 13 & \mathrm{~d} 48\end{array}$
$\begin{array}{lllllll}\mathrm{H} & 24 & \mathrm{r} 49 & 20 & \mathrm{a} 49 & 13 & \mathrm{~d} 49\end{array}$
Variables:

$$
\begin{aligned}
& \mathrm{r} 2=1.4000 \\
& \mathrm{r} 3=1.4072 \\
& \mathrm{a} 3=120.99 \\
& \mathrm{r} 4=1.3920 \\
& \mathrm{a} 4=120.17 \\
& \mathrm{~d} 4=1.21 \\
& \mathrm{r} 5=1.4048 \\
& \mathrm{a} 5=119.46 \\
& \mathrm{~d} 5=359.21 \\
& \mathrm{r} 6=1.4005 \\
& \mathrm{a} 6=119.94 \\
& \mathrm{~d} 6=359.74 \\
& \mathrm{r} 7=1.5231 \\
& \mathrm{a} 7=109.81 \\
& \mathrm{~d} 7=183.73 \\
& \mathrm{r} 8=1.5712 \\
& \mathrm{a} 8=102.31 \\
& \mathrm{~d} 8=355.11 \\
& \mathrm{r} 9=1.3936 \\
& \mathrm{a} 9=128.13 \\
& \mathrm{~d} 9=177.29
\end{aligned}
$$




$$
\begin{aligned}
& \mathrm{r} 10=1.4650 \\
& \mathrm{a} 10=105.15 \\
& \mathrm{~d} 10=242.41 \\
& \mathrm{r} 11=1.4590 \\
& \mathrm{a} 11=112.57 \\
& \mathrm{~d} 11=16.80 \\
& \mathrm{r} 12=1.5430 \\
& \mathrm{a} 12=103.15 \\
& \mathrm{~d} 12=331.90 \\
& \mathrm{r} 13=1.5451 \\
& \mathrm{a} 13=116.02 \\
& \mathrm{~d} 13=95.06 \\
& \mathrm{r} 14=1.3720 \\
& \mathrm{a} 14=120.00 \\
& \mathrm{~d} 14=198.82 \\
& \mathrm{r} 15=1.5556 \\
& \mathrm{a} 15=112.17 \\
& \mathrm{~d} 15=115.92 \\
& \mathrm{~d} 16=1.5035 \\
& \mathrm{a} 16=115.03 \\
& \mathrm{~d} 16=305.95 \\
& \mathrm{r} 17=1.3480 \\
& \mathrm{a} 17=128.16 \\
& \mathrm{~d} 17=242.18 \\
& \mathrm{r} 18=1.5099 \\
& \mathrm{a} 18=120.63 \\
& \mathrm{~d} 18=180.52 \\
& \mathrm{r} 19=1.5088 \\
& \mathrm{a} 19=125.09 \\
& \mathrm{~d} 19=
\end{aligned}
$$




$$
\begin{aligned}
& \mathrm{a} 22=117.07 \\
& \mathrm{~d} 22=177.87 \\
& \mathrm{r} 23=1.2270 \\
& \mathrm{a} 23=120.75 \\
& \mathrm{~d} 23=357.88 \\
& \text { r24= } 1.4496 \\
& \mathrm{a} 24=123.01 \\
& \mathrm{~d} 24=182.01 \\
& \text { r25 }=1.0988 \\
& \mathrm{a} 25=111.03 \\
& \mathrm{~d} 25=214.19 \\
& \text { r26 }=1.0976 \\
& \mathrm{a} 26=114.04 \\
& \mathrm{~d} 26=123.92 \\
& \text { r27 }=1.0925 \\
& \mathrm{a} 27=120.83 \\
& \mathrm{~d} 27=179.67 \\
& \text { r28 }=1.0939 \\
& \mathrm{a} 28=120.51 \\
& \mathrm{~d} 28=179.98 \\
& \text { r29 }=1.0925 \\
& \mathrm{a} 29=120.00 \\
& \mathrm{~d} 29=180.18 \\
& \text { r30 }=1.0934 \\
& \text { a30 }=119.67 \\
& \mathrm{~d} 30=180.54 \\
& \text { r31 }=1.0145 \\
& \text { a31 }=119.93 \\
& \mathrm{~d} 31=34.84 \\
& \text { r32 }=1.1000 \\
& \text { a32 }=108.74 \\
& \mathrm{~d} 32=269.69 \\
& \text { r33 }=1.0980 \\
& \text { a33 }=111.73 \\
& \mathrm{~d} 33=151.10 \\
& \text { r34 }=1.1060 \\
& \text { a34 }=106.90
\end{aligned}
$$




$$
\begin{aligned}
& \mathrm{d} 34=67.43 \\
& \mathrm{r} 35=1.1005 \\
& \mathrm{a} 35=107.88 \\
& \mathrm{~d} 35=181.22 \\
& \mathrm{r} 36=1.0967 \\
& \mathrm{a} 36=114.86 \\
& \mathrm{~d} 36=62.49 \\
& \mathrm{r} 37=1.1048 \\
& \mathrm{a} 37=110.93 \\
& \mathrm{~d} 37=120.57 \\
& \mathrm{r} 38=1.1048 \\
& \mathrm{a} 38=110.92 \\
& \mathrm{~d} 38=238.28 \\
& \mathrm{r} 39=1.1001 \\
& \mathrm{a} 39=112.13 \\
& \mathrm{~d} 39=359.43 \\
& \mathrm{r} 40=1.1047 \\
& \mathrm{a} 40=110.42 \\
& \mathrm{~d} 40=239.38 \\
& \mathrm{~d} 46=116=297.77 \\
& \mathrm{r} 41=1.0978 \\
& \mathrm{a} 41=113.74 \\
& \mathrm{~d} 41=0.81 \\
& \mathrm{r} 42=1.1043 \\
& \mathrm{a} 42=110.43 \\
& \mathrm{~d} 42=122.33 \\
& \mathrm{r} 43=1.0187 \\
& \mathrm{a} 43=117.60 \\
& \mathrm{~d} 43=350.44 \\
& \mathrm{r} 44=1.1007 \\
& \mathrm{a} 44=111.21 \\
& \mathrm{~d} 44=57.66 \\
& \mathrm{~d} 4=1.0965
\end{aligned}
$$


$\mathrm{r} 47=1.1033$

$\mathrm{a} 47=111.47$

$\mathrm{d} 47=106.02$

$\mathrm{r} 48=1.1009$

$\mathrm{a} 48=110.67$

$\mathrm{d} 48=226.72$

$\mathrm{r} 49=1.0973$

a $49=108.49$

$\mathrm{d} 49=346.47$ 


\section{SRR3}

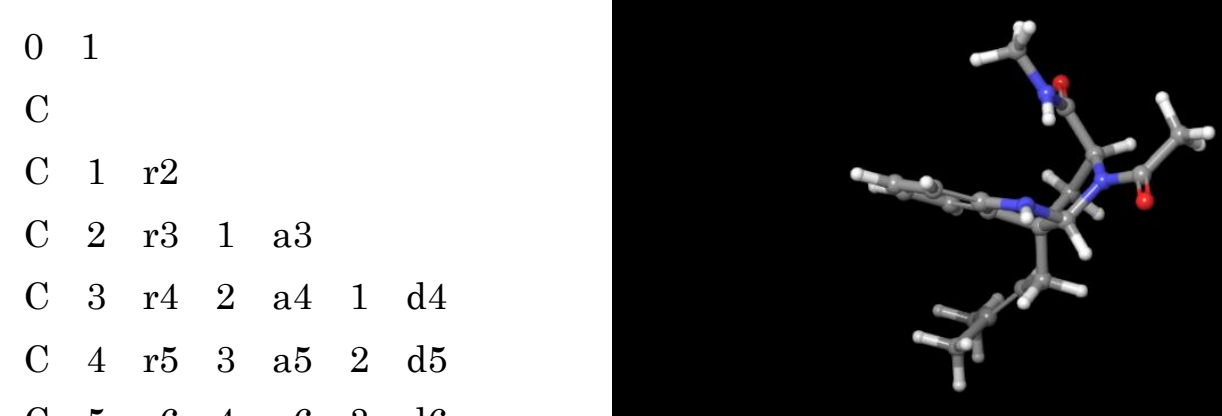

C $5 \begin{array}{llllll}5 & \text { r6 } & 4 & \text { a6 } & 3 & \text { d6 }\end{array}$

$\begin{array}{lllllll}\text { C } & 3 & \text { r7 } & 2 & \text { a7 } & 1 & \text { d7 }\end{array}$

$\begin{array}{lllllll}\mathrm{C} & 7 & \mathrm{r} 8 & 3 & \mathrm{a} 8 & 2 & \mathrm{~d} 8\end{array}$

$\begin{array}{lllllll}\mathrm{N} & 2 & \mathrm{r} 9 & 1 & \mathrm{a} 9 & 3 & \mathrm{~d} 9\end{array}$

$\begin{array}{lllllll}\mathrm{N} & 8 & \mathrm{r} 10 & 7 & \mathrm{a} 10 & 3 & \mathrm{~d} 10\end{array}$

$\begin{array}{lllllll}\text { C } & 10 & \text { r11 } & 8 & \text { a11 } & 7 & \text { d11 }\end{array}$

$\begin{array}{lllllll}\text { C } & 11 & \text { r12 } & 10 & \text { a12 } & 8 & \text { d12 }\end{array}$

$\begin{array}{lllllll}\text { C } & 11 & \text { r13 } & 10 & \text { a13 } & 8 & \text { d13 }\end{array}$

$\begin{array}{lllllll}\text { C } & 10 & \text { r14 } & 8 & \text { a14 } & 7 & \text { d14 }\end{array}$

$\begin{array}{lllllll}\text { C } & 7 & \text { r15 } & 3 & \text { a15 } & 2 & \text { d } 15\end{array}$

$\begin{array}{lllllll}\mathrm{C} & 15 & \mathrm{r} 16 & 7 & \mathrm{a} 16 & 3 & \mathrm{~d} 16\end{array}$

$\begin{array}{lllllll}\text { C } & 16 & \text { r17 } & 15 & \text { a17 } & 7 & \mathrm{~d} 17\end{array}$

$\begin{array}{lllllll}\text { C } & 17 & \text { r18 } & 16 & \text { a18 } & 15 & \text { d18 }\end{array}$

$\begin{array}{lllllll}\text { C } & 17 & \text { r19 } & 16 & \text { a19 } & 15 & \text { d19 }\end{array}$

$\begin{array}{lllllll}\mathrm{N} & 13 & \mathrm{r} 20 & 11 & \mathrm{a} 20 & 10 & \mathrm{~d} 20\end{array}$

$\begin{array}{lllllll}\mathrm{O} & 13 & \mathrm{r} 21 & 11 & \mathrm{a} 21 & 10 & \mathrm{~d} 21\end{array}$

$\begin{array}{lllllll}\text { C } & 14 & \text { r22 } & 10 & \text { a22 } & 8 & \text { d } 22\end{array}$

$\begin{array}{lllllll}\text { O } & 14 & \text { r23 } & 10 & \text { a23 } & 8 & \text { d23 }\end{array}$

$\begin{array}{lllllll}\text { C } & 20 & \text { r24 } & 13 & \text { a24 } & 11 & \text { d24 }\end{array}$

$\begin{array}{lllllll}\mathrm{H} & 11 & \mathrm{r} 25 & 10 & \mathrm{a} 25 & 8 & \mathrm{~d} 25\end{array}$

$\begin{array}{lllllll}\mathrm{H} & 8 & \mathrm{r} 26 & 7 & \mathrm{a} 26 & 3 & \mathrm{~d} 26\end{array}$

$\begin{array}{lllllll}\mathrm{H} & 1 & \mathrm{r} 27 & 2 & \mathrm{a} 27 & 3 & \mathrm{~d} 27\end{array}$

$\begin{array}{lllllll}\mathrm{H} & 4 & \mathrm{r} 28 & 3 & \mathrm{a} 28 & 2 & \mathrm{~d} 28\end{array}$

$\begin{array}{lllllll}\mathrm{H} & 5 & \mathrm{r} 29 & 4 & \mathrm{a} 29 & 3 & \mathrm{~d} 29\end{array}$

$\begin{array}{lllllll}\mathrm{H} & 6 & \text { r30 } & 5 & \text { a330 } & 4 & \text { d } 30\end{array}$

$\begin{array}{lllllll}\mathrm{H} & 9 & \text { r31 } & 2 & \text { a31 } & 1 & \text { d } 31\end{array}$

$\begin{array}{lllllll}\mathrm{H} & 12 & \text { r32 } & 11 & \text { a32 } & 10 & \text { d32 }\end{array}$

$\begin{array}{lllllll}\mathrm{H} & 12 & \text { r33 } & 11 & \text { a33 } & 10 & \text { d } 33\end{array}$

$\begin{array}{lllllll}\mathrm{H} & 15 & \text { r34 } & 7 & \text { a34 } & 3 & \text { d34 }\end{array}$ 

$\begin{array}{lllllll}\mathrm{H} & 15 & \mathrm{r} 35 & 7 & \mathrm{a} 35 & 3 & \mathrm{~d} 35\end{array}$
$\begin{array}{lllllll}\mathrm{H} & 16 & \mathrm{r} 36 & 15 & \text { a36 } & 7 & \mathrm{~d} 36\end{array}$
$\begin{array}{lllllll}\mathrm{H} & 18 & \mathrm{r} 37 & 17 & \mathrm{a} 37 & 16 & \mathrm{~d} 37\end{array}$
$\begin{array}{lllllll}\mathrm{H} & 18 & \mathrm{r} 38 & 17 & \mathrm{a} 38 & 16 & \mathrm{~d} 38\end{array}$
$\begin{array}{lllllll}\mathrm{H} & 18 & \mathrm{r} 39 & 17 & \mathrm{a} 39 & 16 & \mathrm{~d} 39\end{array}$
$\begin{array}{lllllll}\mathrm{H} & 19 & \mathrm{r} 40 & 17 & \mathrm{a} 40 & 16 & \mathrm{~d} 40\end{array}$
$\begin{array}{lllllll}\mathrm{H} & 19 & \mathrm{r} 41 & 17 & \mathrm{a} 41 & 16 & \mathrm{~d} 41\end{array}$
$\begin{array}{lllllll}\mathrm{H} & 19 & \mathrm{r} 42 & 17 & \mathrm{a} 42 & 16 & \mathrm{~d} 42\end{array}$
$\begin{array}{lllllll}\mathrm{H} & 20 & \mathrm{r} 43 & 13 & \mathrm{a} 43 & 11 & \mathrm{~d} 43\end{array}$
$\begin{array}{lllllll}\mathrm{H} & 22 & \mathrm{r} 44 & 14 & \mathrm{a} 44 & 10 & \mathrm{~d} 44\end{array}$
$\begin{array}{lllllll}\mathrm{H} & 22 & \mathrm{r} 45 & 14 & \mathrm{a} 45 & 10 & \mathrm{~d} 45\end{array}$
$\begin{array}{lllllll}\mathrm{H} & 22 & \mathrm{r} 46 & 14 & \mathrm{a} 46 & 10 & \mathrm{~d} 46\end{array}$
$\begin{array}{lllllll}\mathrm{H} & 24 & \mathrm{r} 47 & 20 & \mathrm{a} 47 & 13 & \mathrm{~d} 47\end{array}$
$\begin{array}{lllllll}\mathrm{H} & 24 & \mathrm{r} 48 & 20 & \mathrm{a} 48 & 13 & \mathrm{~d} 48\end{array}$
$\begin{array}{lllllll}\mathrm{H} & 24 & \mathrm{r} 49 & 20 & \mathrm{a} 49 & 13 & \mathrm{~d} 49\end{array}$
Variables:

$$
\begin{aligned}
& \mathrm{r} 2=1.3999 \\
& \mathrm{r} 3=1.4094 \\
& \mathrm{a} 3=121.11 \\
& \mathrm{r} 4=1.3924 \\
& \mathrm{a} 4=119.94 \\
& \mathrm{~d} 4=2.12 \\
& \mathrm{r} 5=1.4056 \\
& \mathrm{a} 5=119.52 \\
& \mathrm{~d} 5=358.52 \\
& \mathrm{r} 6=1.4000 \\
& \mathrm{a} 6=120.00 \\
& \mathrm{~d} 6=359.82 \\
& \mathrm{r} 7=1.5248 \\
& \mathrm{a} 7=109.58 \\
& \mathrm{~d} 7=184.63 \\
& \mathrm{r} 8=1.5724 \\
& \mathrm{a} 8=102.10 \\
& \mathrm{~d} 8=352.12 \\
& \mathrm{r} 9=1.3906 \\
& \mathrm{a} 9=128.08 \\
& \mathrm{~d} 9=177.02
\end{aligned}
$$




$$
\begin{aligned}
& \mathrm{r} 10=1.4679 \\
& \mathrm{a} 10=105.12 \\
& \mathrm{~d} 10=245.83 \\
& \mathrm{r} 11=1.4590 \\
& \mathrm{a} 11=112.54 \\
& \mathrm{~d} 11=13.81 \\
& \mathrm{r} 12=1.5419 \\
& \mathrm{a} 12=103.07 \\
& \mathrm{~d} 12=332.52 \\
& \mathrm{r} 13=1.5449 \\
& \mathrm{a} 13=115.90 \\
& \mathrm{~d} 13=95.58 \\
& \mathrm{r} 14=1.3723 \\
& \mathrm{a} 14=119.80 \\
& \mathrm{~d} 14=198.36 \\
& \mathrm{r} 15=1.5572 \\
& \mathrm{a} 15=111.76 \\
& \mathrm{~d} 15=110.14 \\
& \mathrm{r} 16=1.5030 \\
& \mathrm{a} 16=115.16 \\
& \mathrm{~d} 16=70.66 \\
& \mathrm{r} 22=1.5163 \\
& \mathrm{r} 17=1.3475 \\
& \mathrm{a} 17=128.35 \\
& \mathrm{~d} 17=239.28 \\
& \mathrm{r} 18=1.5098 \\
& \mathrm{a} 18=120.69 \\
& \mathrm{~d} 18=181.08 \\
& \mathrm{r} 19=1.5080 \\
& \mathrm{a} 19=124.94 \\
& \mathrm{~d} 19= \\
& \mathrm{r} 20=1.3510 \\
& \mathrm{~d} 20.69
\end{aligned}
$$


$\mathrm{a} 22=117.14$

$\mathrm{d} 22=176.91$

$\mathrm{r} 23=1.2271$

$\mathrm{a} 23=120.67$

$\mathrm{d} 23=356.85$

r24 $=1.4494$

$\mathrm{a} 24=123.16$

$\mathrm{d} 24=181.72$

r25 $=1.0989$

$\mathrm{a} 25=111.08$

$\mathrm{d} 25=214.84$

r26 $=1.0983$

a26 $=113.94$

$\mathrm{d} 26=127.80$

r27 $=1.0925$

a27 $=120.82$

$\mathrm{d} 27=179.59$

r28 $=1.0920$

$\mathrm{a} 28=120.39$

$\mathrm{d} 28=179.27$

r29 $=1.0926$

a29 $=119.93$

$\mathrm{d} 29=180.18$

r30 $=1.0935$

$\mathrm{a} 30=119.71$

$\mathrm{d} 30=180.58$

r31 $=1.0137$

a31 $=121.08$

$\mathrm{d} 31=32.58$

r32 $=1.1004$

a32 $=108.48$

$\mathrm{d} 32=272.00$

r33 $=1.0977$

a33 $=111.63$

$\mathrm{d} 33=153.46$

r34 $=1.1067$

a34= 106.55 


$$
\begin{aligned}
& \mathrm{d} 34=191.26 \\
& \mathrm{r} 35=1.0997 \\
& \mathrm{a} 35=108.27 \\
& \mathrm{~d} 35=305.37 \\
& \mathrm{r} 36=1.0975 \\
& \mathrm{a} 36=114.88 \\
& \mathrm{~d} 36=60.43 \\
& \mathrm{r} 37=1.1047 \\
& \mathrm{a} 37=110.96 \\
& \mathrm{~d} 37=121.08 \\
& \mathrm{r} 38=1.1048 \\
& \mathrm{a} 38=110.88 \\
& \mathrm{~d} 38=238.79 \\
& \mathrm{r} 39=1.1002 \\
& \mathrm{a} 39=112.16 \\
& \mathrm{~d} 39=359.91 \\
& \mathrm{r} 40=1.1045 \\
& \mathrm{a} 40=110.50 \\
& \mathrm{~d} 40=238.39 \\
& \mathrm{r} 41=1.0978 \\
& \mathrm{a} 41=113.65 \\
& \mathrm{~d} 46=176=176.39 \\
& \mathrm{~d} 41=359.97 \\
& \mathrm{r} 42=1.1043 \\
& \mathrm{a} 42=110.34 \\
& \mathrm{~d} 42=121.27 \\
& \mathrm{r} 43=1.0173 \\
& \mathrm{a} 43=117.57 \\
& \mathrm{~d} 43=351.12 \\
& \mathrm{r} 44=1.1017 \\
& \mathrm{a} 44=111.10 \\
& \mathrm{~d} 44=296.07 \\
& \mathrm{a} 45=1006
\end{aligned}
$$


$\mathrm{r} 47=1.1030$

$\mathrm{a} 47=111.42$

$\mathrm{d} 47=107.07$

$\mathrm{r} 48=1.1010$

$\mathrm{a} 48=110.71$

$\mathrm{d} 48=227.81$

$\mathrm{r} 49=1.0972$

$\mathrm{a} 49=108.45$

$\mathrm{d} 49=347.54$ 


\section{SRR4}
$\begin{array}{ll}0 & 1\end{array}$
$\mathrm{C}$
C 11 r2
C $2 \begin{array}{llll}\text { r } 3 & 1 & \text { a3 }\end{array}$
$\begin{array}{lllllll}\text { C } & 3 & \text { r4 } & 2 & \text { a } 4 & 1 & \mathrm{~d} 4\end{array}$
$\begin{array}{lllllll}\mathrm{C} & 4 & \mathrm{r} 5 & 3 & \mathrm{a} 5 & 2 & \mathrm{~d} 5\end{array}$
$\begin{array}{lllllll}\mathrm{C} & 5 & \mathrm{r} 6 & 4 & \mathrm{a} 6 & 3 & \mathrm{~d} 6\end{array}$
$\begin{array}{lllllll}\text { C } & 3 & \text { r7 } & 2 & \text { a7 } & 1 & \text { d7 }\end{array}$
$\begin{array}{lllllll}\mathrm{C} & 7 & \mathrm{r} 8 & 3 & \mathrm{a} 8 & 2 & \mathrm{~d} 8\end{array}$
$\begin{array}{lllllll}\mathrm{N} & 2 & \mathrm{r} 9 & 1 & \mathrm{a} 9 & 3 & \mathrm{~d} 9\end{array}$
$\begin{array}{lllllll}\mathrm{N} & 8 & \mathrm{r} 10 & 7 & \mathrm{a} 10 & 3 & \mathrm{~d} 10\end{array}$
$\begin{array}{lllllll}\text { C } & 10 & \text { r11 } & 8 & \text { a11 } & 7 & \text { d11 }\end{array}$
$\begin{array}{lllllll}\text { C } & 11 & \text { r12 } & 10 & \text { a12 } & 8 & \text { d12 }\end{array}$
$\begin{array}{lllllll}\text { C } & 11 & \text { r13 } & 10 & \text { a13 } & 8 & \text { d } 13\end{array}$
$\begin{array}{lllllll}\text { C } & 10 & \text { r14 } & 8 & \text { a14 } & 7 & \text { d14 }\end{array}$
$\begin{array}{lllllll}\text { C } & 7 & \text { r15 } & 3 & \text { a15 } & 2 & \text { d } 15\end{array}$
$\begin{array}{lllllll}\mathrm{C} & 15 & \mathrm{r} 16 & 7 & \mathrm{a} 16 & 3 & \mathrm{~d} 16\end{array}$
$\begin{array}{lllllll}\text { C } & 16 & \text { r17 } & 15 & \text { a17 } & 7 & \text { d } 17\end{array}$
$\begin{array}{lllllll}\text { C } & 17 & \text { r18 } & 16 & \text { a18 } & 15 & \text { d18 }\end{array}$
$\begin{array}{lllllll}\text { C } & 17 & \text { r19 } & 16 & \text { a19 } & 15 & \text { d19 }\end{array}$
$\begin{array}{lllllll}\mathrm{N} & 13 & \mathrm{r} 20 & 11 & \mathrm{a} 20 & 10 & \mathrm{~d} 20\end{array}$
$\begin{array}{lllllll}\mathrm{O} & 13 & \mathrm{r} 21 & 11 & \mathrm{a} 21 & 10 & \mathrm{~d} 21\end{array}$
$\begin{array}{lllllll}\text { C } & 14 & \text { r22 } & 10 & \text { a22 } & 8 & \text { d22 }\end{array}$
$\begin{array}{lllllll}\text { O } & 14 & \text { r23 } & 10 & \text { a23 } & 8 & \text { d23 }\end{array}$
$\begin{array}{lllllll}\text { C } & 20 & \text { r24 } & 13 & \text { a24 } & 11 & \text { d24 }\end{array}$
$\begin{array}{lllllll}\mathrm{H} & 11 & \mathrm{r} 25 & 10 & \mathrm{a} 25 & 8 & \mathrm{~d} 25\end{array}$
$\begin{array}{lllllll}\mathrm{H} & 8 & \mathrm{r} 26 & 7 & \mathrm{a} 26 & 3 & \mathrm{~d} 26\end{array}$
$\begin{array}{lllllll}\mathrm{H} & 1 & \mathrm{r} 27 & 2 & \mathrm{a} 27 & 3 & \mathrm{~d} 27\end{array}$
$\begin{array}{lllllll}\mathrm{H} & 4 & \mathrm{r} 28 & 3 & \mathrm{a} 28 & 2 & \mathrm{~d} 28\end{array}$
$\begin{array}{lllllll}\mathrm{H} & 5 & \mathrm{r} 29 & 4 & \mathrm{a} 29 & 3 & \mathrm{~d} 29\end{array}$
$\begin{array}{lllllll}\mathrm{H} & 6 & \mathrm{r} 30 & 5 & \text { a330 } & 4 & \mathrm{~d} 30\end{array}$
$\begin{array}{lllllll}\mathrm{H} & 9 & \text { r31 } & 2 & \text { a31 } & 1 & \text { d } 31\end{array}$
$\begin{array}{lllllll}\mathrm{H} & 12 & \text { r32 } & 11 & \text { a32 } & 10 & \text { d } 32\end{array}$
$\begin{array}{lllllll}\mathrm{H} & 12 & \text { r33 } & 11 & \text { a33 } & 10 & \text { d } 33\end{array}$
$\begin{array}{lllllll}\mathrm{H} & 15 & \mathrm{r} 34 & 7 & \mathrm{a} 34 & 3 & \mathrm{~d} 34\end{array}$






$\begin{array}{lllllll}\mathrm{H} & 15 & \mathrm{r} 35 & 7 & \mathrm{a} 35 & 3 & \mathrm{~d} 35 \\ \mathrm{H} & 16 & \mathrm{r} 36 & 15 & \mathrm{a} 36 & 7 & \mathrm{~d} 36 \\ \mathrm{H} & 18 & \mathrm{r} 37 & 17 & \mathrm{a} 37 & 16 & \mathrm{~d} 37 \\ \mathrm{H} & 18 & \mathrm{r} 38 & 17 & \mathrm{a} 38 & 16 & \mathrm{~d} 38 \\ \mathrm{H} & 18 & \mathrm{r} 39 & 17 & \mathrm{a} 39 & 16 & \mathrm{~d} 39 \\ \mathrm{H} & 19 & \mathrm{r} 40 & 17 & \mathrm{a} 40 & 16 & \mathrm{~d} 40 \\ \mathrm{H} & 19 & \mathrm{r} 41 & 17 & \mathrm{a} 41 & 16 & \mathrm{~d} 41 \\ \mathrm{H} & 19 & \mathrm{r} 42 & 17 & \mathrm{a} 42 & 16 & \mathrm{~d} 42 \\ \mathrm{H} & 20 & \mathrm{r} 43 & 13 & \mathrm{a} 43 & 11 & \mathrm{~d} 43 \\ \mathrm{H} & 22 & \mathrm{r} 44 & 14 & \mathrm{a} 44 & 10 & \mathrm{~d} 44 \\ \mathrm{H} & 22 & \mathrm{r} 45 & 14 & \mathrm{a} 45 & 10 & \mathrm{~d} 45 \\ \mathrm{H} & 22 & \mathrm{r} 46 & 14 & \mathrm{a} 46 & 10 & \mathrm{~d} 46 \\ \mathrm{H} & 24 & \mathrm{r} 47 & 20 & \mathrm{a} 47 & 13 & \mathrm{~d} 47 \\ \mathrm{H} & 24 & \mathrm{r} 48 & 20 & \mathrm{a} 48 & 13 & \mathrm{~d} 48 \\ \mathrm{H} & 24 & \mathrm{r} 49 & 20 & \mathrm{a} 49 & 13 & \mathrm{~d} 49 \\ \text { Variables: } & & & & \end{array}$

$\mathrm{r} 2=1.3994$

$\mathrm{r} 3=1.4083$

$\mathrm{a} 3=121.11$

$\mathrm{r} 4=1.3895$

$\mathrm{a} 4=120.19$

$\mathrm{d} 4=359.00$

r5 $=1.4059$

$\mathrm{a} 5=119.41$

$\mathrm{d} 5=0.69$

r6 $=1.4001$

$\mathrm{a} 6=119.96$

$\mathrm{d} 6=359.91$

$\mathrm{r} 7=1.5267$

$a 7=109.10$

$\mathrm{d} 7=182.01$

$\mathrm{r} 8=1.5653$

$\mathrm{a} 8=101.40$

$\mathrm{d} 8=347.24$

$\mathrm{r} 9=1.3888$

$\mathrm{a} 9=129.00$

$\mathrm{d} 9=182.34$ 


$$
\begin{aligned}
& \mathrm{r} 10=1.4827 \\
& \mathrm{a} 10=104.33 \\
& \mathrm{~d} 10=258.51 \\
& \mathrm{r} 11=1.4777 \\
& \mathrm{a} 11=113.10 \\
& \mathrm{~d} 11=359.52 \\
& \mathrm{r} 12=1.5304 \\
& \mathrm{a} 12=103.65 \\
& \mathrm{~d} 12=343.08 \\
& \mathrm{r} 13=1.5535 \\
& \mathrm{a} 13=114.94 \\
& \mathrm{~d} 13=107.52 \\
& \mathrm{r} 14=1.3627 \\
& \mathrm{a} 14=124.77 \\
& \mathrm{~d} 14=192.37 \\
& \mathrm{r} 15=1.5592 \\
& \mathrm{a} 15=109.21 \\
& \mathrm{~d} 15=106.50 \\
& \mathrm{r} 16=1.5054 \\
& \mathrm{a} 16=116.26 \\
& \mathrm{~d} 16=186.78 \\
& \mathrm{r} 22=17=1.5154 \\
& \mathrm{a} 17=127.75 \\
& \mathrm{~d} 17=107.99 \\
& \mathrm{r} 18=1.5095 \\
& \mathrm{a} 18=120.78 \\
& \mathrm{~d} 18=178.63 \\
& \mathrm{r} 19=1.5089 \\
& \mathrm{a} 19=124.82 \\
& \mathrm{~d} 19=359.27 \\
& \mathrm{a} 20=1.3512 \\
& \mathrm{~d} 2019
\end{aligned}
$$




$$
\begin{aligned}
& \mathrm{a} 22=118.23 \\
& \mathrm{~d} 22=357.33 \\
& \mathrm{r} 23=1.2361 \\
& \mathrm{a} 23=121.12 \\
& \mathrm{~d} 23=178.08 \\
& \text { r24= } 1.4481 \\
& \text { a24= } 123.36 \\
& \mathrm{~d} 24=177.80 \\
& \mathrm{r} 25=1.1024 \\
& \text { a } 25=107.55 \\
& \mathrm{~d} 25=224.79 \\
& \text { r26 }=1.0985 \\
& \mathrm{a} 26=112.84 \\
& \mathrm{~d} 26=140.65 \\
& \text { r27 }=1.0926 \\
& \mathrm{a} 27=121.01 \\
& \mathrm{~d} 27=180.59 \\
& \text { r28 }=1.0936 \\
& \mathrm{a} 28=120.54 \\
& \mathrm{~d} 28=179.99 \\
& \text { r29 }=1.0925 \\
& \mathrm{a} 29=119.99 \\
& \mathrm{~d} 29=179.51 \\
& \text { r30 }=1.0937 \\
& \text { a30 }=119.64 \\
& \mathrm{~d} 30=179.59 \\
& \text { r31 }=1.0126 \\
& \mathrm{a} 31=121.20 \\
& \mathrm{~d} 31=341.81 \\
& \text { r32 }=1.0986 \\
& \text { a32 }=109.05 \\
& \mathrm{~d} 32=269.02 \\
& \text { r33 }=1.0969 \\
& \mathrm{a} 33=110.94 \\
& \mathrm{~d} 33=149.49 \\
& \text { r34= } 1.0999 \\
& \text { a34 }=107.65
\end{aligned}
$$




$$
\begin{aligned}
& \mathrm{d} 34=311.83 \\
& \mathrm{r} 35=1.1042 \\
& \mathrm{a} 35=106.48 \\
& \mathrm{~d} 35=65.12 \\
& \mathrm{r} 36=1.0967 \\
& \mathrm{a} 36=115.60 \\
& \mathrm{~d} 36=286.83 \\
& \mathrm{r} 37=1.1046 \\
& \mathrm{a} 37=110.94 \\
& \mathrm{~d} 37=121.52 \\
& \mathrm{r} 38=1.1048 \\
& \mathrm{a} 38=110.85 \\
& \mathrm{~d} 38=239.21 \\
& \mathrm{r} 39=1.1001 \\
& \mathrm{a} 39=112.15 \\
& \mathrm{~d} 39= \\
& \mathrm{r} 40=1.1040 \\
& \mathrm{a} 40=110.32 \\
& \mathrm{~d} 46=112.65 \\
& \mathrm{~d} 40=235.30 \\
& \mathrm{r} 41=1.0979 \\
& \mathrm{a} 41=113.70 \\
& \mathrm{~d} 45=356.57 \\
& \mathrm{r} 42=1.1046 \\
& \mathrm{a} 42=110.49 \\
& \mathrm{~d} 42=118.28 \\
& \mathrm{r} 43=1.0230 \\
& \mathrm{a} 43=116.60 \\
& \mathrm{~d} 43= \\
& \mathrm{r} 44=1.0969 \\
& \mathrm{a} 44=107.96 \\
& \mathrm{~d} 44=170.73 \\
& \mathrm{~d} 4=1096
\end{aligned}
$$


$\mathrm{r} 47=1.1019$

$\mathrm{a} 47=110.90$

$\mathrm{d} 47=129.14$

$\mathrm{r} 48=1.1038$

$\mathrm{a} 48=111.63$

$\mathrm{d} 48=249.76$

$\mathrm{r} 49=1.0973$

a49= 108.69

$\mathrm{d} 49=9.36$ 


\section{SRR5}

\section{$\begin{array}{ll}0 & 1\end{array}$}

C

C 11 r2

C $2 \begin{array}{llll}\text { r } 3 & 1 & \text { a3 }\end{array}$

$\begin{array}{lllllll}\text { C } & 3 & \text { r4 } & 2 & \text { a } 4 & 1 & \mathrm{~d} 4\end{array}$

$\begin{array}{lllllll}\mathrm{C} & 4 & \mathrm{r} 5 & 3 & \mathrm{a} 5 & 2 & \mathrm{~d} 5\end{array}$

$\begin{array}{lllllll}\mathrm{C} & 5 & \mathrm{r} 6 & 4 & \mathrm{a} 6 & 3 & \mathrm{~d} 6\end{array}$

$\begin{array}{lllllll}\text { C } & 3 & \text { r7 } & 2 & \text { a7 } & 1 & \text { d7 }\end{array}$

$\begin{array}{lllllll}\mathrm{C} & 7 & \mathrm{r} 8 & 3 & \mathrm{a} 8 & 2 & \mathrm{~d} 8\end{array}$

$\begin{array}{lllllll}\mathrm{N} & 2 & \mathrm{r} 9 & 1 & \mathrm{a} 9 & 3 & \mathrm{~d} 9\end{array}$

$\begin{array}{lllllll}\mathrm{N} & 8 & \mathrm{r} 10 & 7 & \mathrm{a} 10 & 3 & \mathrm{~d} 10\end{array}$

$\begin{array}{lllllll}\text { C } & 10 & \text { r11 } & 8 & \text { a11 } & 7 & \text { d11 }\end{array}$

$\begin{array}{lllllll}\text { C } & 11 & \text { r12 } & 10 & \text { a12 } & 8 & \text { d12 }\end{array}$

$\begin{array}{lllllll}\text { C } & 11 & \text { r13 } & 10 & \text { a13 } & 8 & \text { d13 }\end{array}$

$\begin{array}{lllllll}\text { C } & 10 & \text { r14 } & 8 & \text { a14 } & 7 & \text { d14 }\end{array}$

$\begin{array}{lllllll}\text { C } & 7 & \text { r15 } & 3 & \text { a15 } & 2 & \text { d15 }\end{array}$

$\begin{array}{lllllll}\mathrm{C} & 15 & \mathrm{r} 16 & 7 & \mathrm{a} 16 & 3 & \mathrm{~d} 16\end{array}$

$\begin{array}{lllllll}\text { C } & 16 & \text { r17 } & 15 & \text { a17 } & 7 & \text { d17 }\end{array}$

$\begin{array}{lllllll}\text { C } & 17 & \text { r18 } & 16 & \text { a18 } & 15 & \text { d18 }\end{array}$

$\begin{array}{lllllll}\text { C } & 17 & \text { r19 } & 16 & \text { a19 } & 15 & \text { d19 }\end{array}$

$\begin{array}{lllllll}\mathrm{N} & 13 & \mathrm{r} 20 & 11 & \mathrm{a} 20 & 10 & \mathrm{~d} 20\end{array}$

$\begin{array}{lllllll}\mathrm{O} & 13 & \mathrm{r} 21 & 11 & \mathrm{a} 21 & 10 & \mathrm{~d} 21\end{array}$

$\begin{array}{lllllll}\text { C } & 14 & \text { r22 } & 10 & \text { a22 } & 8 & \text { d22 }\end{array}$

$\begin{array}{lllllll}\text { O } & 14 & \text { r23 } & 10 & \text { a23 } & 8 & \text { d23 }\end{array}$

$\begin{array}{lllllll}\text { C } & 20 & \text { r24 } & 13 & \text { a24 } & 11 & \text { d24 }\end{array}$

$\begin{array}{lllllll}\mathrm{H} & 11 & \mathrm{r} 25 & 10 & \mathrm{a} 25 & 8 & \mathrm{~d} 25\end{array}$

$\begin{array}{lllllll}\mathrm{H} & 8 & \mathrm{r} 26 & 7 & \mathrm{a} 26 & 3 & \mathrm{~d} 26\end{array}$

$\begin{array}{lllllll}\mathrm{H} & 1 & \mathrm{r} 27 & 2 & \mathrm{a} 27 & 3 & \mathrm{~d} 27\end{array}$

$\begin{array}{lllllll}\mathrm{H} & 4 & \mathrm{r} 28 & 3 & \mathrm{a} 28 & 2 & \mathrm{~d} 28\end{array}$

$\begin{array}{lllllll}\mathrm{H} & 5 & \mathrm{r} 29 & 4 & \mathrm{a} 29 & 3 & \mathrm{~d} 29\end{array}$

$\begin{array}{lllllll}\mathrm{H} & 6 & \mathrm{r} 30 & 5 & \text { a330 } & 4 & \mathrm{~d} 30\end{array}$

$\begin{array}{lllllll}\mathrm{H} & 9 & \text { r31 } & 2 & \text { a31 } & 1 & \text { d } 31\end{array}$

$\begin{array}{lllllll}\mathrm{H} & 12 & \text { r32 } & 11 & \text { a32 } & 10 & \text { d32 }\end{array}$

$\begin{array}{lllllll}\mathrm{H} & 12 & \text { r33 } & 11 & \text { a33 } & 10 & \text { d33 }\end{array}$

$\begin{array}{lllllll}\mathrm{H} & 15 & \text { r34 } & 7 & \text { a34 } & 3 & \text { d34 }\end{array}$ 


$\begin{array}{lllllll}\mathrm{H} & 15 & \mathrm{r} 35 & 7 & \mathrm{a} 35 & 3 & \mathrm{~d} 35 \\ \mathrm{H} & 16 & \mathrm{r} 36 & 15 & \mathrm{a} 36 & 7 & \mathrm{~d} 36 \\ \mathrm{H} & 18 & \mathrm{r} 37 & 17 & \mathrm{a} 37 & 16 & \mathrm{~d} 37 \\ \mathrm{H} & 18 & \mathrm{r} 38 & 17 & \mathrm{a} 38 & 16 & \mathrm{~d} 38 \\ \mathrm{H} & 18 & \mathrm{r} 39 & 17 & \mathrm{a} 39 & 16 & \mathrm{~d} 39 \\ \mathrm{H} & 19 & \mathrm{r} 40 & 17 & \mathrm{a} 40 & 16 & \mathrm{~d} 40 \\ \mathrm{H} & 19 & \mathrm{r} 41 & 17 & \mathrm{a} 41 & 16 & \mathrm{~d} 41 \\ \mathrm{H} & 19 & \mathrm{r} 42 & 17 & \mathrm{a} 42 & 16 & \mathrm{~d} 42 \\ \mathrm{H} & 20 & \mathrm{r} 43 & 13 & \mathrm{a} 43 & 11 & \mathrm{~d} 43 \\ \mathrm{H} & 22 & \mathrm{r} 44 & 14 & \mathrm{a} 44 & 10 & \mathrm{~d} 44 \\ \mathrm{H} & 22 & \mathrm{r} 45 & 14 & \mathrm{a} 45 & 10 & \mathrm{~d} 45 \\ \mathrm{H} & 22 & \mathrm{r} 46 & 14 & \mathrm{a} 46 & 10 & \mathrm{~d} 46 \\ \mathrm{H} & 24 & \mathrm{r} 47 & 20 & \mathrm{a} 47 & 13 & \mathrm{~d} 47 \\ \mathrm{H} & 24 & \mathrm{r} 48 & 20 & \mathrm{a} 48 & 13 & \mathrm{~d} 48 \\ \mathrm{H} & 24 & \mathrm{r} 49 & 20 & \mathrm{a} 49 & 13 & \mathrm{~d} 49 \\ \text { Variables: } & & & & \end{array}$

$\mathrm{r} 2=1.3991$

$\mathrm{r} 3=1.4096$

a3 $=121.32$

$\mathrm{r} 4=1.3909$

$\mathrm{a} 4=119.83$

$\mathrm{d} 4=0.29$

r $5=1.4059$

$\mathrm{a} 5=119.55$

$\mathrm{d} 5=359.54$

r6 $=1.3997$

a6 $=120.04$

$\mathrm{d} 6=0.24$

$\mathrm{r} 7=1.5292$

$\mathrm{a} 7=108.94$

$\mathrm{d} 7=181.80$

$\mathrm{r} 8=1.5669$

a $8=101.24$

$\mathrm{d} 8=346.70$

$\mathrm{r} 9=1.3883$

$\mathrm{a} 9=128.71$

$\mathrm{d} 9=181.70$ 


$$
\begin{aligned}
& \mathrm{r} 10=1.4831 \\
& \mathrm{a} 10=104.40 \\
& \mathrm{~d} 10=258.81 \\
& \mathrm{r} 11=1.4784 \\
& \mathrm{a} 11=113.00 \\
& \mathrm{~d} 11=358.39 \\
& \mathrm{r} 12=1.5306 \\
& \mathrm{a} 12=103.78 \\
& \mathrm{~d} 12=344.06 \\
& \mathrm{r} 13=1.5538 \\
& \mathrm{a} 13=115.00 \\
& \mathrm{~d} 13=108.46 \\
& \text { r14 }=1.3628 \\
& \text { a14 }=124.75 \\
& \text { d14= } 191.74 \\
& \mathrm{r} 15=1.5569 \\
& \mathrm{a} 15=112.09 \\
& \mathrm{~d} 15=104.25 \\
& \text { r16 }=1.5035 \\
& \text { a16 }=115.19 \\
& \mathrm{~d} 16=67.25 \\
& \text { r17 }=1.3479 \\
& \mathrm{a} 17=128.22 \\
& \mathrm{~d} 17=114.88 \\
& \mathrm{r} 18=1.5099 \\
& \text { a18 }=120.63 \\
& \mathrm{~d} 18=179.12 \\
& \mathrm{r} 19=1.5087 \\
& \mathrm{a} 19=125.12 \\
& \mathrm{~d} 19=359.50 \\
& \mathrm{r} 20=1.3511 \\
& \mathrm{a} 20=114.13 \\
& \mathrm{~d} 20=54.15 \\
& \mathrm{r} 21=1.2279 \\
& \mathrm{a} 21=121.74 \\
& \mathrm{~d} 21=231.45 \\
& \mathrm{r} 22=1.5155
\end{aligned}
$$




$$
\begin{aligned}
& \mathrm{a} 22=118.27 \\
& \mathrm{~d} 22=356.35 \\
& \mathrm{r} 23=1.2360 \\
& \mathrm{a} 23=121.14 \\
& \mathrm{~d} 23=177.33 \\
& \text { r24= } 1.4482 \\
& \text { a24= } 123.30 \\
& \mathrm{~d} 24=177.71 \\
& \mathrm{r} 25=1.1024 \\
& \text { a } 25=107.58 \\
& \mathrm{~d} 25=225.74 \\
& \text { r26 }=1.0996 \\
& \mathrm{a} 26=112.76 \\
& \mathrm{~d} 26=140.89 \\
& \text { r27 }=1.0927 \\
& \text { a27= } 120.94 \\
& \mathrm{~d} 27=180.41 \\
& \text { r28 }=1.0924 \\
& \mathrm{a} 28=120.55 \\
& \mathrm{~d} 28=178.53 \\
& \text { r29 }=1.0926 \\
& \mathrm{a} 29=119.91 \\
& \mathrm{~d} 29=179.74 \\
& \text { r30 }=1.0935 \\
& \mathrm{a} 30=119.70 \\
& \mathrm{~d} 30=179.65 \\
& \text { r31 }=1.0125 \\
& \mathrm{a} 31=121.21 \\
& \mathrm{~d} 31=342.71 \\
& \text { r32 }=1.1001 \\
& \text { a32 }=109.43 \\
& \mathrm{~d} 32=269.10 \\
& \text { r33 }=1.0962 \\
& \text { a33 }=110.82 \\
& \mathrm{~d} 33=149.64 \\
& \text { r34= } 1.1007 \\
& \text { a34 }=107.68
\end{aligned}
$$




$$
\begin{aligned}
& \mathrm{d} 34=191.50 \\
& \text { r35 }=1.1049 \\
& \text { a35 }=107.24 \\
& \text { d } 35=305.73 \\
& \text { r36 }=1.0960 \\
& \text { a36 }=114.97 \\
& \mathrm{~d} 36=293.80 \\
& \text { r37 }=1.1049 \\
& \text { a } 37=110.94 \\
& \mathrm{~d} 37=121.78 \\
& \text { r38 }=1.1049 \\
& \mathrm{a} 38=110.95 \\
& \mathrm{~d} 38=239.49 \\
& \text { r39 }=1.1002 \\
& \text { a39 }=112.15 \\
& \mathrm{~d} 39=0.63 \\
& \mathrm{r} 40=1.1045 \\
& \mathrm{a} 40=110.43 \\
& \mathrm{~d} 40=239.17 \\
& \mathrm{r} 41=1.0978 \\
& \mathrm{a} 41=113.72 \\
& \mathrm{~d} 41=\quad 0.61 \\
& \mathrm{r} 42=1.1045 \\
& \mathrm{a} 42=110.44 \\
& \mathrm{~d} 42=122.11 \\
& \mathrm{r} 43=1.0230 \\
& \mathrm{a} 43=116.61 \\
& \mathrm{~d} 43=4.22 \\
& \mathrm{r} 44=1.0966 \\
& \text { a44= } 107.95 \\
& \mathrm{~d} 44=169.51 \\
& \mathrm{r} 45=1.1016 \\
& \mathrm{a} 45=110.46 \\
& \mathrm{~d} 45=287.51 \\
& \mathrm{r} 46=1.0996 \\
& \mathrm{a} 46=112.87 \\
& \mathrm{~d} 46=48.38
\end{aligned}
$$


$\mathrm{r} 47=1.0974$

$\mathrm{a} 47=108.75$

$\mathrm{d} 47=11.78$

$\mathrm{r} 48=1.1017$

$\mathrm{a} 48=110.76$

$\mathrm{d} 48=131.57$

$\mathrm{r} 49=1.1040$

$\mathrm{a} 49=111.73$

$\mathrm{d} 49=252.16$ 


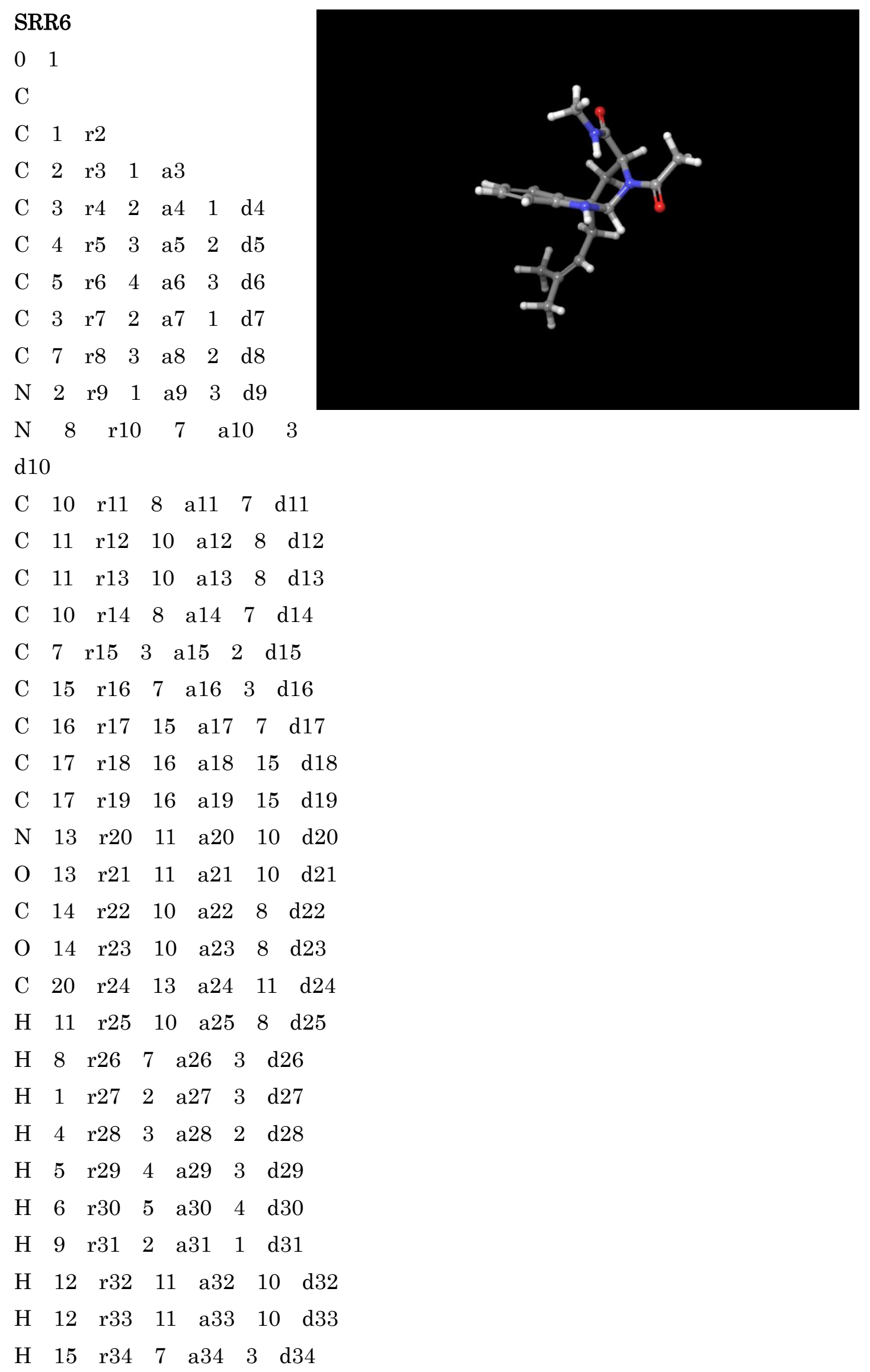




$\begin{array}{lllllll}\mathrm{H} & 15 & \mathrm{r} 35 & 7 & \mathrm{a} 35 & 3 & \mathrm{~d} 35 \\ \mathrm{H} & 16 & \mathrm{r} 36 & 15 & \mathrm{a} 36 & 7 & \mathrm{~d} 36 \\ \mathrm{H} & 18 & \mathrm{r} 37 & 17 & \mathrm{a} 37 & 16 & \mathrm{~d} 37 \\ \mathrm{H} & 18 & \mathrm{r} 38 & 17 & \mathrm{a} 38 & 16 & \mathrm{~d} 38 \\ \mathrm{H} & 18 & \mathrm{r} 39 & 17 & \mathrm{a} 39 & 16 & \mathrm{~d} 39 \\ \mathrm{H} & 19 & \mathrm{r} 40 & 17 & \mathrm{a} 40 & 16 & \mathrm{~d} 40 \\ \mathrm{H} & 19 & \mathrm{r} 41 & 17 & \mathrm{a} 41 & 16 & \mathrm{~d} 41 \\ \mathrm{H} & 19 & \mathrm{r} 42 & 17 & \mathrm{a} 42 & 16 & \mathrm{~d} 42 \\ \mathrm{H} & 20 & \mathrm{r} 43 & 13 & \mathrm{a} 43 & 11 & \mathrm{~d} 43 \\ \mathrm{H} & 22 & \mathrm{r} 44 & 14 & \mathrm{a} 44 & 10 & \mathrm{~d} 44 \\ \mathrm{H} & 22 & \mathrm{r} 45 & 14 & \mathrm{a} 45 & 10 & \mathrm{~d} 45 \\ \mathrm{H} & 22 & \mathrm{r} 46 & 14 & \mathrm{a} 46 & 10 & \mathrm{~d} 46 \\ \mathrm{H} & 24 & \mathrm{r} 47 & 20 & \mathrm{a} 47 & 13 & \mathrm{~d} 47 \\ \mathrm{H} & 24 & \mathrm{r} 48 & 20 & \mathrm{a} 48 & 13 & \mathrm{~d} 48 \\ \mathrm{H} & 24 & \mathrm{r} 49 & 20 & \mathrm{a} 49 & 13 & \mathrm{~d} 49 \\ \text { Variables: } & & & & \end{array}$

$\mathrm{r} 2=1.3999$

$\mathrm{r} 3=1.4071$

a3 $=121.03$

$\mathrm{r} 4=1.3917$

$\mathrm{a} 4=120.13$

$\mathrm{d} 4=1.31$

r $5=1.4050$

$\mathrm{a} 5=119.48$

$\mathrm{d} 5=359.22$

r6 $=1.4004$

a6 $=119.94$

$\mathrm{d} 6=359.66$

$\mathrm{r} 7=1.5229$

$\mathrm{a} 7=109.80$

$\mathrm{d} 7=184.00$

$\mathrm{r} 8=1.5733$

$\mathrm{a} 8=102.23$

$\mathrm{d} 8=354.15$

$\mathrm{r} 9=1.3928$

$\mathrm{a} 9=128.13$

$\mathrm{d} 9=177.32$ 


$$
\begin{aligned}
& \mathrm{r} 10=1.4662 \\
& \mathrm{a} 10=105.11 \\
& \mathrm{~d} 10=243.65 \\
& \mathrm{r} 11=1.4587 \\
& \mathrm{a} 11=112.63 \\
& \mathrm{~d} 11=15.89 \\
& \mathrm{r} 12=1.5421 \\
& \mathrm{a} 12=103.04 \\
& \mathrm{~d} 12=332.01 \\
& \mathrm{r} 13=1.5450 \\
& \mathrm{a} 13=116.00 \\
& \mathrm{~d} 13=95.17 \\
& \mathrm{r} 14=1.3722 \\
& \mathrm{a} 14=119.84 \\
& \mathrm{~d} 14=198.93 \\
& \mathrm{r} 15=1.5551 \\
& \mathrm{a} 15=111.97 \\
& \mathrm{~d} 15=115.33 \\
& \mathrm{r} 16=1.5037 \\
& \mathrm{a} 16=115.31 \\
& \mathrm{~d} 16=305.36 \\
& \mathrm{r} 17=1.3471 \\
& \mathrm{a} 17=128.46 \\
& \mathrm{~d} 17=122.37 \\
& \mathrm{r} 18=1.5098 \\
& \mathrm{a} 18=120.70 \\
& \mathrm{~d} 18=178.93 \\
& \mathrm{r} 19=1.5083 \\
& \mathrm{a} 19=124.97 \\
& \mathrm{~d} 19=359.26 \\
& \mathrm{r} 20=1.3510 \\
& \mathrm{~d} 20=116.66 \\
& \mathrm{~d} 21=34.97
\end{aligned}
$$


$\mathrm{a} 22=117.14$

$\mathrm{d} 22=177.44$

$\mathrm{r} 23=1.2271$

a23 $=120.71$

$\mathrm{d} 23=357.46$

r24= 1.4494

$\mathrm{a} 24=123.05$

$\mathrm{d} 24=181.77$

r25 $=1.0989$

$\mathrm{a} 25=111.04$

$\mathrm{d} 25=214.36$

r26 $=1.0980$

$\mathrm{a} 26=114.48$

$\mathrm{d} 26=125.61$

r27 $=1.0925$

a27 $=120.85$

$\mathrm{d} 27=179.63$

r28 $=1.0938$

$\mathrm{a} 28=120.50$

$\mathrm{d} 28=180.01$

r29= 1.0926

a29 $=120.00$

$\mathrm{d} 29=180.14$

r30 $=1.0935$

$\mathrm{a} 30=119.68$

$\mathrm{d} 30=180.59$

r31 $=1.0143$

a31 $=120.40$

$\mathrm{d} 31=33.79$

r32 $=1.1001$

a32 $=108.67$

$\mathrm{d} 32=270.63$

r33 $=1.0981$

$\mathrm{a} 33=111.80$

$\mathrm{d} 33=151.91$

r34 $=1.1005$

$\mathrm{a} 34=107.88$ 


$$
\begin{aligned}
& \mathrm{d} 34=70.38 \\
& \text { r35 }=1.1064 \\
& \text { a35 }=106.79 \\
& \mathrm{~d} 35=184.09 \\
& \text { r36 }=1.0975 \\
& \text { a36 }=114.92 \\
& \text { d36 }=301.16 \\
& \text { r37 }=1.1047 \\
& \text { a } 37=110.91 \\
& \mathrm{~d} 37=121.53 \\
& \text { r38 }=1.1049 \\
& \text { a38 }=110.96 \\
& \mathrm{~d} 38=239.25 \\
& \text { r39 }=1.1002 \\
& \text { a39 }=112.15 \\
& \mathrm{~d} 39=\quad 0.40 \\
& \mathrm{r} 40=1.1045 \\
& \mathrm{a} 40=110.46 \\
& \mathrm{~d} 40=120.66 \\
& \mathrm{r} 41=1.1042 \\
& \mathrm{a} 41=110.39 \\
& \mathrm{~d} 41=237.77 \\
& \mathrm{r} 42=1.0979 \\
& \mathrm{a} 42=113.68 \\
& \mathrm{~d} 42=359.18 \\
& \mathrm{r} 43=1.0181 \\
& \mathrm{a} 43=117.57 \\
& \mathrm{~d} 43=350.61 \\
& \mathrm{r} 44=1.1014 \\
& \mathrm{a} 44=111.18 \\
& \mathrm{~d} 44=297.28 \\
& \mathrm{r} 45=1.1007 \\
& \mathrm{a} 45=111.25 \\
& \mathrm{~d} 45=57.22 \\
& \mathrm{r} 46=1.0967 \\
& \mathrm{a} 46=108.36 \\
& \mathrm{~d} 46=177.43
\end{aligned}
$$


$\mathrm{r} 47=1.0972$

$\mathrm{a} 47=108.44$

$\mathrm{d} 47=347.35$

$\mathrm{r} 48=1.1031$

$\mathrm{a} 48=111.46$

$\mathrm{d} 48=106.90$

$\mathrm{r} 49=1.1011$

$\mathrm{a} 49=110.71$

$\mathrm{d} 49=227.63$ 


\section{SRR7}
$\begin{array}{ll}0 & 1\end{array}$
C
C 11 r2
C $2 \begin{array}{llll}\text { r } 3 & 1 & \text { a3 }\end{array}$
$\begin{array}{lllllll}\text { C } & 3 & \text { r4 } & 2 & \text { a } 4 & 1 & \mathrm{~d} 4\end{array}$
$\begin{array}{lllllll}\mathrm{C} & 4 & \text { r5 } & 3 & \text { a5 } & 2 & \text { d5 }\end{array}$
C $5 \begin{array}{llllll}5 & \mathrm{r} 6 & 4 & \mathrm{a} 6 & 3 & \mathrm{~d} 6\end{array}$
$\begin{array}{lllllll}\text { C } & 3 & \text { r7 } & 2 & \text { a7 } & 1 & \text { d7 }\end{array}$
$\begin{array}{lllllll}\mathrm{C} & 7 & \mathrm{r} 8 & 3 & \mathrm{a} 8 & 2 & \mathrm{~d} 8\end{array}$
$\begin{array}{lllllll}\mathrm{N} & 2 & \mathrm{r} 9 & 1 & \mathrm{a} 9 & 3 & \mathrm{~d} 9\end{array}$
$\begin{array}{lllllll}\mathrm{N} & 8 & \mathrm{r} 10 & 7 & \mathrm{a} 10 & 3 & \mathrm{~d} 10\end{array}$
$\begin{array}{lllllll}\text { C } & 10 & \text { r11 } & 8 & \text { a11 } & 7 & \text { d11 }\end{array}$
$\begin{array}{lllllll}\text { C } & 11 & \text { r12 } & 10 & \text { a12 } & 8 & \text { d12 }\end{array}$
$\begin{array}{lllllll}\text { C } & 11 & \text { r13 } & 10 & \text { a13 } & 8 & \text { d13 }\end{array}$
$\begin{array}{lllllll}\text { C } & 10 & \text { r14 } & 8 & \text { a14 } & 7 & \text { d14 }\end{array}$
$\begin{array}{lllllll}\text { C } & 7 & \text { r15 } & 3 & \text { a15 } & 2 & \text { d15 }\end{array}$
$\begin{array}{lllllll}\text { C } & 15 & \text { r16 } & 7 & \text { a16 } & 3 & \text { d16 }\end{array}$
$\begin{array}{lllllll}\mathrm{C} & 16 & \mathrm{r} 17 & 15 & \text { a17 } & 7 & \mathrm{~d} 17\end{array}$
$\begin{array}{lllllll}\text { C } & 17 & \text { r18 } & 16 & \text { a18 } & 15 & \text { d18 }\end{array}$
$\begin{array}{lllllll}\text { C } & 17 & \text { r19 } & 16 & \text { a19 } & 15 & \text { d19 }\end{array}$
$\begin{array}{lllllll}\mathrm{N} & 13 & \mathrm{r} 20 & 11 & \mathrm{a} 20 & 10 & \mathrm{~d} 20\end{array}$
$\begin{array}{lllllll}\mathrm{O} & 13 & \mathrm{r} 21 & 11 & \mathrm{a} 21 & 10 & \mathrm{~d} 21\end{array}$
$\begin{array}{lllllll}\text { C } & 14 & \text { r22 } & 10 & \text { a22 } & 8 & \text { d } 22\end{array}$
$\begin{array}{lllllll}\mathrm{O} & 14 & \text { r23 } & 10 & \text { a23 } & 8 & \text { d23 }\end{array}$
$\begin{array}{lllllll}\text { C } & 20 & \text { r24 } & 13 & \text { a24 } & 11 & \text { d24 }\end{array}$
$\begin{array}{lllllll}\mathrm{H} & 11 & \mathrm{r} 25 & 10 & \mathrm{a} 25 & 8 & \mathrm{~d} 25\end{array}$
$\begin{array}{lllllll}\mathrm{H} & 8 & \mathrm{r} 26 & 7 & \mathrm{a} 26 & 3 & \mathrm{~d} 26\end{array}$
$\begin{array}{lllllll}\mathrm{H} & 1 & \mathrm{r} 27 & 2 & \mathrm{a} 27 & 3 & \mathrm{~d} 27\end{array}$
$\begin{array}{lllllll}\mathrm{H} & 4 & \mathrm{r} 28 & 3 & \mathrm{a} 28 & 2 & \mathrm{~d} 28\end{array}$
$\begin{array}{lllllll}\mathrm{H} & 5 & \mathrm{r} 29 & 4 & \mathrm{a} 29 & 3 & \mathrm{~d} 29\end{array}$
$\begin{array}{lllllll}\mathrm{H} & 6 & \mathrm{r} 30 & 5 & \text { a330 } & 4 & \mathrm{~d} 30\end{array}$
$\begin{array}{lllllll}\mathrm{H} & 9 & \text { r31 } & 2 & \text { a31 } & 1 & \text { d } 31\end{array}$
$\begin{array}{lllllll}\mathrm{H} & 12 & \text { r32 } & 11 & \text { a32 } & 10 & \text { d32 }\end{array}$
$\begin{array}{lllllll}\mathrm{H} & 12 & \text { r33 } & 11 & \text { a33 } & 10 & \text { d33 }\end{array}$
$\begin{array}{lllllll}\mathrm{H} & 15 & \mathrm{r} 34 & 7 & \mathrm{a} 34 & 3 & \mathrm{~d} 34\end{array}$ 


$\begin{array}{lllllll}\mathrm{H} & 15 & \mathrm{r} 35 & 7 & \mathrm{a} 35 & 3 & \mathrm{~d} 35 \\ \mathrm{H} & 16 & \mathrm{r} 36 & 15 & \mathrm{a} 36 & 7 & \mathrm{~d} 36 \\ \mathrm{H} & 18 & \mathrm{r} 37 & 17 & \mathrm{a} 37 & 16 & \mathrm{~d} 37 \\ \mathrm{H} & 18 & \mathrm{r} 38 & 17 & \mathrm{a} 38 & 16 & \mathrm{~d} 38 \\ \mathrm{H} & 18 & \mathrm{r} 39 & 17 & \mathrm{a} 39 & 16 & \mathrm{~d} 39 \\ \mathrm{H} & 19 & \mathrm{r} 40 & 17 & \mathrm{a} 40 & 16 & \mathrm{~d} 40 \\ \mathrm{H} & 19 & \mathrm{r} 41 & 17 & \mathrm{a} 41 & 16 & \mathrm{~d} 41 \\ \mathrm{H} & 19 & \mathrm{r} 42 & 17 & \mathrm{a} 42 & 16 & \mathrm{~d} 42 \\ \mathrm{H} & 20 & \mathrm{r} 43 & 13 & \mathrm{a} 43 & 11 & \mathrm{~d} 43 \\ \mathrm{H} & 22 & \mathrm{r} 44 & 14 & \mathrm{a} 44 & 10 & \mathrm{~d} 44 \\ \mathrm{H} & 22 & \mathrm{r} 45 & 14 & \mathrm{a} 45 & 10 & \mathrm{~d} 45 \\ \mathrm{H} & 22 & \mathrm{r} 46 & 14 & \mathrm{a} 46 & 10 & \mathrm{~d} 46 \\ \mathrm{H} & 24 & \mathrm{r} 47 & 20 & \mathrm{a} 47 & 13 & \mathrm{~d} 47 \\ \mathrm{H} & 24 & \mathrm{r} 48 & 20 & \mathrm{a} 48 & 13 & \mathrm{~d} 48 \\ \mathrm{H} & 24 & \mathrm{r} 49 & 20 & \mathrm{a} 49 & 13 & \mathrm{~d} 49 \\ \text { Variables: } & & & & \end{array}$

$\mathrm{r} 2=1.3991$

$\mathrm{r} 3=1.4059$

$\mathrm{a} 3=121.08$

$\mathrm{r} 4=1.3925$

$\mathrm{a} 4=120.14$

$\mathrm{d} 4=\quad 1.16$

r $5=1.4044$

$\mathrm{a} 5=119.41$

$\mathrm{d} 5=359.28$

r6 $=1.4006$

$\mathrm{a} 6=119.99$

$\mathrm{d} 6=359.69$

$\mathrm{r} 7=1.5225$

$\mathrm{a} 7=109.89$

$\mathrm{d} 7=184.41$

$\mathrm{r} 8=1.5756$

$\mathrm{a} 8=102.14$

$\mathrm{d} 8=353.13$

$\mathrm{r} 9=1.3973$

$\mathrm{a} 9=127.91$

$\mathrm{d} 9=177.54$ 


$$
\begin{aligned}
& \mathrm{r} 10=1.4670 \\
& \mathrm{a} 10=104.73 \\
& \mathrm{~d} 10=244.85 \\
& \mathrm{r} 11=1.4592 \\
& \mathrm{a} 11=112.67 \\
& \mathrm{~d} 11=15.16 \\
& \mathrm{r} 12=1.5395 \\
& \mathrm{a} 12=103.32 \\
& \mathrm{~d} 12=332.22 \\
& \mathrm{r} 13=1.5424 \\
& \mathrm{a} 13=115.78 \\
& \mathrm{~d} 13=96.18 \\
& \mathrm{r} 14=1.3732 \\
& \mathrm{a} 14=126.37 \\
& \mathrm{~d} 14=200.79 \\
& \mathrm{r} 15=1.5598 \\
& \mathrm{a} 15=109.58 \\
& \mathrm{~d} 15=112.74 \\
& \mathrm{r} 16=1.5045 \\
& \mathrm{a} 16=115.77 \\
& \mathrm{~d} 16=188.97 \\
& \mathrm{r} 17=1.3488 \\
& \mathrm{a} 17=127.91 \\
& \mathrm{~d} 17=113.70 \\
& \mathrm{r} 18=1.5098 \\
& \mathrm{a} 18=120.65 \\
& \mathrm{~d} 18=179.09 \\
& \mathrm{r} 19=1.5086 \\
& \mathrm{a} 19=125.05 \\
& \mathrm{~d} 19=359.85
\end{aligned}
$$




$$
\begin{aligned}
& \mathrm{a} 22=116.96 \\
& \mathrm{~d} 22=357.58 \\
& \mathrm{r} 23=1.2261 \\
& \mathrm{a} 23=121.11 \\
& \mathrm{~d} 23=177.70 \\
& \text { r24 }=1.4486 \\
& \text { a24= } 123.29 \\
& \mathrm{~d} 24=181.24 \\
& \text { r25 }=1.0987 \\
& \mathrm{a} 25=109.07 \\
& \mathrm{~d} 25=213.53 \\
& \text { r26 }=1.0972 \\
& \mathrm{a} 26=112.50 \\
& \mathrm{~d} 26=126.14 \\
& \text { r27 }=1.0925 \\
& \mathrm{a} 27=120.85 \\
& \mathrm{~d} 27=179.78 \\
& \text { r28 }=1.0937 \\
& \mathrm{a} 28=120.56 \\
& \mathrm{~d} 28=179.93 \\
& \text { r29 }=1.0925 \\
& \mathrm{a} 29=119.97 \\
& \mathrm{~d} 29=180.08 \\
& \text { r30 }=1.0934 \\
& \mathrm{a} 30=119.68 \\
& \mathrm{~d} 30=180.50 \\
& \text { r31 }=1.0150 \\
& \mathrm{a} 31=118.43 \\
& \mathrm{~d} 31=38.55 \\
& \text { r32 }=1.0984 \\
& \text { a32 }=108.63 \\
& \mathrm{~d} 32=270.02 \\
& \text { r33 }=1.0982 \\
& \text { a33 }=111.93 \\
& \mathrm{~d} 33=151.45 \\
& \text { r34= } 1.0997 \\
& \text { a34 }=107.80
\end{aligned}
$$




$$
\begin{aligned}
& \mathrm{d} 34=313.75 \\
& \text { r35 }=1.1049 \\
& \text { a35 }=106.66 \\
& \mathrm{~d} 35=67.23 \\
& \text { r36 }=1.0966 \\
& \text { a36 }=115.58 \\
& \text { d } 36=292.76 \\
& \text { r37 }=1.1046 \\
& \text { a } 37=110.91 \\
& \mathrm{~d} 37=122.01 \\
& \text { r38 }=1.1048 \\
& \text { a38 }=110.86 \\
& \mathrm{~d} 38=239.69 \\
& \text { r39 }=1.1000 \\
& \text { a39 }=112.16 \\
& \mathrm{~d} 39=\quad 0.81 \\
& \mathrm{r} 40=1.1045 \\
& \mathrm{a} 40=110.38 \\
& \mathrm{~d} 40=120.30 \\
& \mathrm{r} 41=1.1042 \\
& \mathrm{a} 41=110.41 \\
& \mathrm{~d} 41=237.29 \\
& \mathrm{r} 42=1.0979 \\
& \mathrm{a} 42=113.76 \\
& \mathrm{~d} 42=358.83 \\
& \mathrm{r} 43=1.0171 \\
& \mathrm{a} 43=117.69 \\
& \mathrm{~d} 43=353.38 \\
& \text { r44 }=1.1005 \\
& \mathrm{a} 44=111.35 \\
& \mathrm{~d} 44=301.47 \\
& \mathrm{r} 45=1.1013 \\
& \mathrm{a} 45=111.38 \\
& \mathrm{~d} 45=61.68 \\
& \text { r46 }=1.0965 \\
& \mathrm{a} 46=108.25 \\
& \mathrm{~d} 46=181.51
\end{aligned}
$$


$\mathrm{r} 47=1.0973$

$\mathrm{a} 47=108.39$

$\mathrm{d} 47=352.61$

$\mathrm{r} 48=1.1030$

$\mathrm{a} 48=111.32$

$\mathrm{d} 48=112.14$

$\mathrm{r} 49=1.1018$

$\mathrm{a} 49=110.98$

$\mathrm{d} 49=232.89$ 


\section{SRR8}
$\begin{array}{ll}0 & 1\end{array}$
$\mathrm{C}$
C 11 r2
C $2 \begin{array}{llll}\text { r } 3 & 1 & \text { a3 }\end{array}$
$\begin{array}{lllllll}\text { C } & 3 & \text { r4 } & 2 & \text { a } 4 & 1 & \mathrm{~d} 4\end{array}$
$\begin{array}{lllllll}\mathrm{C} & 4 & \mathrm{r} 5 & 3 & \mathrm{a} 5 & 2 & \mathrm{~d} 5\end{array}$
$\begin{array}{lllllll}\mathrm{C} & 5 & \mathrm{r} 6 & 4 & \mathrm{a} 6 & 3 & \mathrm{~d} 6\end{array}$
$\begin{array}{lllllll}\mathrm{C} & 3 & \mathrm{r} 7 & 2 & \mathrm{a} 7 & 1 & \mathrm{~d} 7\end{array}$
$\begin{array}{lllllll}\mathrm{C} & 7 & \mathrm{r} 8 & 3 & \mathrm{a} 8 & 2 & \mathrm{~d} 8\end{array}$
$\begin{array}{lllllll}\mathrm{N} & 2 & \mathrm{r} 9 & 1 & \mathrm{a} 9 & 3 & \mathrm{~d} 9\end{array}$
$\begin{array}{lllllll}\mathrm{N} & 8 & \mathrm{r} 10 & 7 & \mathrm{a} 10 & 3 & \mathrm{~d} 10\end{array}$
$\begin{array}{lllllll}\text { C } & 10 & \text { r11 } & 8 & \text { a11 } & 7 & \text { d11 }\end{array}$
$\begin{array}{lllllll}\text { C } & 11 & \text { r12 } & 10 & \text { a12 } & 8 & \text { d12 }\end{array}$
$\begin{array}{lllllll}\text { C } & 11 & \text { r13 } & 10 & \text { a13 } & 8 & \text { d } 13\end{array}$
$\begin{array}{lllllll}\text { C } & 10 & \text { r14 } & 8 & \text { a14 } & 7 & \text { d14 }\end{array}$
$\begin{array}{lllllll}\text { C } & 7 & \text { r15 } & 3 & \text { a15 } & 2 & \text { d15 }\end{array}$
$\begin{array}{lllllll}\mathrm{C} & 15 & \mathrm{r} 16 & 7 & \mathrm{a} 16 & 3 & \mathrm{~d} 16\end{array}$
$\begin{array}{lllllll}\text { C } & 16 & \text { r17 } & 15 & \text { a17 } & 7 & \text { d17 }\end{array}$
$\begin{array}{lllllll}\text { C } & 17 & \text { r18 } & 16 & \text { a18 } & 15 & \text { d18 }\end{array}$
$\begin{array}{lllllll}\mathrm{C} & 17 & \mathrm{r} 19 & 16 & \mathrm{a} 19 & 15 & \mathrm{~d} 19\end{array}$
$\begin{array}{lllllll}\mathrm{N} & 13 & \mathrm{r} 20 & 11 & \mathrm{a} 20 & 10 & \mathrm{~d} 20\end{array}$
$\begin{array}{lllllll}\mathrm{O} & 13 & \mathrm{r} 21 & 11 & \mathrm{a} 21 & 10 & \mathrm{~d} 21\end{array}$
$\begin{array}{lllllll}\text { C } & 14 & \text { r22 } & 10 & \text { a22 } & 8 & \text { d22 }\end{array}$
$\begin{array}{lllllll}\mathrm{O} & 14 & \mathrm{r} 23 & 10 & \text { a23 } & 8 & \mathrm{~d} 23\end{array}$
$\begin{array}{lllllll}\text { C } & 20 & \text { r24 } & 13 & \text { a24 } & 11 & \text { d24 }\end{array}$
$\begin{array}{lllllll}\mathrm{H} & 11 & \mathrm{r} 25 & 10 & \mathrm{a} 25 & 8 & \mathrm{~d} 25\end{array}$
$\begin{array}{lllllll}\mathrm{H} & 8 & \mathrm{r} 26 & 7 & \mathrm{a} 26 & 3 & \mathrm{~d} 26\end{array}$
$\begin{array}{lllllll}\mathrm{H} & 1 & \mathrm{r} 27 & 2 & \mathrm{a} 27 & 3 & \mathrm{~d} 27\end{array}$
$\begin{array}{lllllll}\mathrm{H} & 4 & \mathrm{r} 28 & 3 & \mathrm{a} 28 & 2 & \mathrm{~d} 28\end{array}$
$\begin{array}{lllllll}\mathrm{H} & 5 & \mathrm{r} 29 & 4 & \mathrm{a} 29 & 3 & \mathrm{~d} 29\end{array}$
$\begin{array}{lllllll}\mathrm{H} & 6 & \mathrm{r} 30 & 5 & \mathrm{a} 30 & 4 & \mathrm{~d} 30\end{array}$
$\begin{array}{lllllll}\mathrm{H} & 9 & \text { r31 } & 2 & \text { a31 } & 1 & \mathrm{~d} 31\end{array}$
$\begin{array}{lllllll}\mathrm{H} & 12 & \text { r32 } & 11 & \text { a32 } & 10 & \text { d } 32\end{array}$
$\begin{array}{lllllll}\mathrm{H} & 12 & \text { r33 } & 11 & \text { a33 } & 10 & \text { d33 }\end{array}$
$\begin{array}{lllllll}\mathrm{H} & 15 & \text { r34 } & 7 & \text { a34 } & 3 & \text { d34 }\end{array}$ 

$\begin{array}{lllllll}\mathrm{H} & 15 & \mathrm{r} 35 & 7 & \mathrm{a} 35 & 3 & \mathrm{~d} 35\end{array}$
$\begin{array}{lllllll}\mathrm{H} & 16 & \mathrm{r} 36 & 15 & \text { a36 } & 7 & \mathrm{~d} 36\end{array}$
$\begin{array}{lllllll}\mathrm{H} & 18 & \mathrm{r} 37 & 17 & \mathrm{a} 37 & 16 & \mathrm{~d} 37\end{array}$
$\begin{array}{lllllll}\mathrm{H} & 18 & \mathrm{r} 38 & 17 & \mathrm{a} 38 & 16 & \mathrm{~d} 38\end{array}$
$\begin{array}{lllllll}\mathrm{H} & 18 & \mathrm{r} 39 & 17 & \mathrm{a} 39 & 16 & \mathrm{~d} 39\end{array}$
$\begin{array}{lllllll}\mathrm{H} & 19 & \mathrm{r} 40 & 17 & \mathrm{a} 40 & 16 & \mathrm{~d} 40\end{array}$
$\begin{array}{lllllll}\mathrm{H} & 19 & \mathrm{r} 41 & 17 & \mathrm{a} 41 & 16 & \mathrm{~d} 41\end{array}$
$\begin{array}{lllllll}\mathrm{H} & 19 & \mathrm{r} 42 & 17 & \mathrm{a} 42 & 16 & \mathrm{~d} 42\end{array}$
$\begin{array}{lllllll}\mathrm{H} & 20 & \mathrm{r} 43 & 13 & \mathrm{a} 43 & 11 & \mathrm{~d} 43\end{array}$
$\begin{array}{lllllll}\mathrm{H} & 22 & \mathrm{r} 44 & 14 & \mathrm{a} 44 & 10 & \mathrm{~d} 44\end{array}$
$\begin{array}{lllllll}\mathrm{H} & 22 & \mathrm{r} 45 & 14 & \mathrm{a} 45 & 10 & \mathrm{~d} 45\end{array}$
$\begin{array}{lllllll}\mathrm{H} & 22 & \mathrm{r} 46 & 14 & \mathrm{a} 46 & 10 & \mathrm{~d} 46\end{array}$
$\begin{array}{lllllll}\mathrm{H} & 24 & \mathrm{r} 47 & 20 & \mathrm{a} 47 & 13 & \mathrm{~d} 47\end{array}$
$\begin{array}{lllllll}\mathrm{H} & 24 & \mathrm{r} 48 & 20 & \mathrm{a} 48 & 13 & \mathrm{~d} 48\end{array}$
$\begin{array}{lllllll}\mathrm{H} & 24 & \mathrm{r} 49 & 20 & \mathrm{a} 49 & 13 & \mathrm{~d} 49\end{array}$
Variables:

$$
\begin{aligned}
& \mathrm{r} 2=1.3993 \\
& \mathrm{r} 3=1.4124 \\
& \mathrm{a} 3=121.07 \\
& \mathrm{r} 4=1.3910 \\
& \mathrm{a} 4=119.99 \\
& \mathrm{~d} 4=0.57 \\
& \mathrm{r} 5=1.4068 \\
& \mathrm{a} 5=119.52 \\
& \mathrm{~d} 5=359.48 \\
& \mathrm{r} 6=1.3993 \\
& \mathrm{a} 6=119.98 \\
& \mathrm{~d} 6=0.06 \\
& \mathrm{r} 7=1.5295 \\
& \mathrm{a} 7=108.84 \\
& \mathrm{~d} 7=181.54 \\
& \mathrm{r} 8=1.5618 \\
& \mathrm{a} 8=100.88 \\
& \mathrm{~d} 8=345.76 \\
& \mathrm{r} 9=1.3883 \\
& \mathrm{a} 9=128.86 \\
& \mathrm{~d} 9=181.04
\end{aligned}
$$




$$
\begin{aligned}
& \mathrm{r} 10=1.4820 \\
& \mathrm{a} 10=104.37 \\
& \mathrm{~d} 10=260.96 \\
& \mathrm{r} 11=1.4650 \\
& \mathrm{a} 11=113.06 \\
& \mathrm{~d} 11=358.13 \\
& \mathrm{r} 12=1.5407 \\
& \mathrm{a} 12=103.53 \\
& \mathrm{~d} 12=342.93 \\
& \mathrm{r} 13=1.5454 \\
& \mathrm{a} 13=116.16 \\
& \mathrm{~d} 13=106.10 \\
& \mathrm{r} 14=1.3740 \\
& \mathrm{a} 14=118.28 \\
& \mathrm{~d} 14=192.54 \\
& \mathrm{r} 15=1.5559 \\
& \mathrm{a} 15=112.40 \\
& \mathrm{~d} 15=103.76 \\
& \mathrm{r} 16=1.5036 \\
& \mathrm{a} 16=115.10 \\
& \mathrm{~d} 16=67.32 \\
& \mathrm{r} 17=1.3480 \\
& \mathrm{a} 17=128.21 \\
& \mathrm{~d} 17=114.79 \\
& \mathrm{r} 18=1.5100 \\
& \mathrm{a} 18=120.64 \\
& \mathrm{~d} 18=179.21 \\
& \mathrm{r} 19=1.5086 \\
& \mathrm{a} 19=125.11 \\
& \mathrm{~d} 19=359.59 \\
& \mathrm{a} 20=1.3507 \\
& \mathrm{~d} 20.36
\end{aligned}
$$




$$
\begin{aligned}
& \mathrm{a} 22=117.57 \\
& \mathrm{~d} 22=170.25 \\
& \mathrm{r} 23=1.2278 \\
& \mathrm{a} 23=120.20 \\
& \mathrm{~d} 23=350.19 \\
& \text { r24= } 1.4494 \\
& \mathrm{a} 24=123.47 \\
& \mathrm{~d} 24=180.68 \\
& \mathrm{r} 25=1.1003 \\
& \mathrm{a} 25=110.90 \\
& \mathrm{~d} 25=225.21 \\
& \text { r26 }=1.0994 \\
& \mathrm{a} 26=114.29 \\
& \mathrm{~d} 26=143.17 \\
& \text { r27 }=1.0926 \\
& \mathrm{a} 27=120.87 \\
& \mathrm{~d} 27=180.31 \\
& \text { r28 }=1.0929 \\
& \text { a28 }=120.61 \\
& \mathrm{~d} 28=178.78 \\
& \text { r29 }=1.0926 \\
& \mathrm{a} 29=119.92 \\
& \mathrm{~d} 29=179.79 \\
& \text { r30 }=1.0936 \\
& \text { a30 }=119.65 \\
& \mathrm{~d} 30=179.85 \\
& \text { r31 }=1.0139 \\
& \mathrm{a} 31=121.82 \\
& \mathrm{~d} 31=338.53 \\
& \text { r32 }=1.1006 \\
& \mathrm{a} 32=109.20 \\
& \mathrm{~d} 32=271.68 \\
& \text { r33 }=1.0972 \\
& \mathrm{a} 33=111.50 \\
& \mathrm{~d} 33=152.80 \\
& \text { r34= } 1.1006 \\
& \text { a34= } 107.64
\end{aligned}
$$




$$
\begin{aligned}
& \mathrm{d} 34=191.63 \\
& \mathrm{r} 35=1.1048 \\
& \mathrm{a} 35=107.21 \\
& \mathrm{~d} 35=305.78 \\
& \mathrm{r} 36=1.0962 \\
& \mathrm{a} 36=114.95 \\
& \mathrm{~d} 36=293.83 \\
& \mathrm{r} 37=1.1048 \\
& \mathrm{a} 37=110.94 \\
& \mathrm{~d} 37=121.75 \\
& \mathrm{r} 38=1.1049 \\
& \mathrm{a} 38=110.94 \\
& \mathrm{~d} 38=239.47 \\
& \mathrm{r} 39=1.1002 \\
& \mathrm{a} 39=112.15 \\
& \mathrm{~d} 39= \\
& \mathrm{r} 40=1.1043 \\
& \mathrm{a} 40=110.44 \\
& \mathrm{~d} 46=116=51.78 \\
& \mathrm{~d} 40=239.07 \\
& \mathrm{r} 41=1.0979 \\
& \mathrm{a} 41=113.72 \\
& \mathrm{~d} 41=0.52 \\
& \mathrm{r} 42=1.1044 \\
& \mathrm{a} 42=110.42 \\
& \mathrm{~d} 42=122.01 \\
& \mathrm{r} 43=1.0139 \\
& \mathrm{a} 43=117.46 \\
& \mathrm{~d} 43=352.57 \\
& \mathrm{r} 44=1.0966 \\
& \mathrm{~d} 44=108.43 \\
& \mathrm{~d} 45=172.73 \\
& \mathrm{a}=1020
\end{aligned}
$$


$\mathrm{r} 47=1.1011$

$\mathrm{a} 47=110.79$

$\mathrm{d} 47=230.31$

$\mathrm{r} 48=1.0971$

$\mathrm{a} 48=108.27$

$\mathrm{d} 48=349.96$

$\mathrm{r} 49=1.1025$

$\mathrm{a} 49=111.33$

$\mathrm{d} 49=109.46$ 


\section{SRR11}
$\begin{array}{ll}0 & 1\end{array}$
C
C 1 r 2
C $2 \begin{array}{llll}\text { r } 3 & 1 & \text { a3 }\end{array}$
$\begin{array}{lllllll}\text { C } & 3 & \text { r } 4 & 2 & \text { a } 4 & 1 & \text { d } 4\end{array}$
$\begin{array}{lllllll}\mathrm{C} & 4 & \mathrm{r} 5 & 3 & \mathrm{a} 5 & 2 & \mathrm{~d} 5\end{array}$
C $\quad 5 \quad$ r6 $64 \begin{array}{llll}\text { a6 } & 3 & \text { d6 }\end{array}$

$\begin{array}{lllllll}\mathrm{C} & 3 & \mathrm{r} 7 & 2 & \mathrm{a} 7 & 1 & \mathrm{~d} 7\end{array}$

$\begin{array}{lllllll}\mathrm{C} & 7 & \mathrm{r} 8 & 3 & \mathrm{a} 8 & 2 & \mathrm{~d} 8\end{array}$

$\begin{array}{lllllll}\mathrm{N} & 2 & \mathrm{r} 9 & 1 & \mathrm{a} 9 & 3 & \mathrm{~d} 9\end{array}$

$\begin{array}{lllllll}\mathrm{N} & 8 & \mathrm{r} 10 & 7 & \mathrm{a} 10 & 3 & \mathrm{~d} 10\end{array}$

$\begin{array}{lllllll}\text { C } & 10 & \text { r11 } & 8 & \text { a11 } & 7 & \text { d11 }\end{array}$

$\begin{array}{lllllll}\text { C } & 11 & \text { r12 } & 10 & \text { a12 } & 8 & \text { d12 }\end{array}$

$\begin{array}{lllllll}\text { C } & 11 & \text { r13 } & 10 & \text { a13 } & 8 & \text { d13 }\end{array}$

$\begin{array}{lllllll}\text { C } & 10 & \text { r14 } & 8 & \text { a14 } & 7 & \text { d14 }\end{array}$

$\begin{array}{lllllll}\text { C } & 7 & \text { r15 } & 3 & \text { a15 } & 2 & \text { d15 }\end{array}$

$\begin{array}{lllllll}\text { C } & 15 & \text { r16 } & 7 & \text { a16 } & 3 & \text { d16 }\end{array}$

$\begin{array}{lllllll}\text { C } & 16 & \text { r17 } & 15 & \text { a17 } & 7 & \text { d17 }\end{array}$

$\begin{array}{lllllll}\text { C } & 17 & \text { r18 } & 16 & \text { a18 } & 15 & \text { d18 }\end{array}$

$\begin{array}{lllllll}\text { C } & 17 & \text { r19 } & 16 & \text { a19 } & 15 & \text { d19 }\end{array}$

$\begin{array}{lllllll}\mathrm{N} & 13 & \mathrm{r} 20 & 11 & \mathrm{a} 20 & 10 & \mathrm{~d} 20\end{array}$

$\begin{array}{lllllll}\mathrm{O} & 13 & \mathrm{r} 21 & 11 & \mathrm{a} 21 & 10 & \mathrm{~d} 21\end{array}$

$\begin{array}{lllllll}\mathrm{C} & 14 & \mathrm{r} 22 & 10 & \mathrm{a} 22 & 8 & \mathrm{~d} 22\end{array}$

$\begin{array}{lllllll}\mathrm{O} & 14 & \mathrm{r} 23 & 10 & \mathrm{a} 23 & 8 & \mathrm{~d} 23\end{array}$

$\begin{array}{lllllll}\text { C } & 20 & \text { r24 } & 13 & \text { a24 } & 11 & \text { d } 24\end{array}$

$\begin{array}{lllllll}\mathrm{H} & 11 & \mathrm{r} 25 & 10 & \mathrm{a} 25 & 8 & \mathrm{~d} 25\end{array}$

$\begin{array}{lllllll}\mathrm{H} & 8 & \mathrm{r} 26 & 7 & \mathrm{a} 26 & 3 & \mathrm{~d} 26\end{array}$

$\begin{array}{lllllll}\mathrm{H} & 1 & \mathrm{r} 27 & 2 & \mathrm{a} 27 & 3 & \mathrm{~d} 27\end{array}$

$\begin{array}{lllllll}\mathrm{H} & 4 & \mathrm{r} 28 & 3 & \mathrm{a} 28 & 2 & \mathrm{~d} 28\end{array}$

$\begin{array}{lllllll}\mathrm{H} & 5 & \mathrm{r} 29 & 4 & \mathrm{a} 29 & 3 & \mathrm{~d} 29\end{array}$

$\begin{array}{lllllll}\mathrm{H} & 6 & \mathrm{r} 30 & 5 & \mathrm{a} 30 & 4 & \mathrm{~d} 30\end{array}$

$\begin{array}{lllllll}\mathrm{H} & 9 & \mathrm{r} 31 & 2 & \text { a31 } & 1 & \mathrm{~d} 31\end{array}$

$\begin{array}{lllllll}\mathrm{H} & 12 & \text { r32 } & 11 & \text { a32 } & 10 & \text { d } 32\end{array}$

$\begin{array}{lllllll}\mathrm{H} & 12 & \text { r33 } & 11 & \text { a33 } & 10 & \text { d } 33\end{array}$

$\begin{array}{lllllll}\mathrm{H} & 15 & \mathrm{r} 34 & 7 & \mathrm{a} 34 & 3 & \mathrm{~d} 34\end{array}$

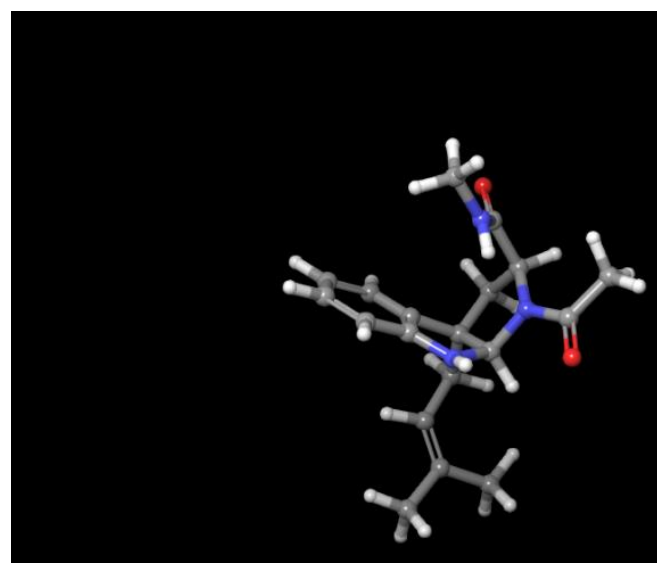




$\begin{array}{lllllll}\mathrm{H} & 15 & \mathrm{r} 35 & 7 & \mathrm{a} 35 & 3 & \mathrm{~d} 35 \\ \mathrm{H} & 16 & \mathrm{r} 36 & 15 & \mathrm{a} 36 & 7 & \mathrm{~d} 36 \\ \mathrm{H} & 18 & \mathrm{r} 37 & 17 & \mathrm{a} 37 & 16 & \mathrm{~d} 37 \\ \mathrm{H} & 18 & \mathrm{r} 38 & 17 & \mathrm{a} 38 & 16 & \mathrm{~d} 38 \\ \mathrm{H} & 18 & \mathrm{r} 39 & 17 & \mathrm{a} 39 & 16 & \mathrm{~d} 39 \\ \mathrm{H} & 19 & \mathrm{r} 40 & 17 & \mathrm{a} 40 & 16 & \mathrm{~d} 40 \\ \mathrm{H} & 19 & \mathrm{r} 41 & 17 & \mathrm{a} 41 & 16 & \mathrm{~d} 41 \\ \mathrm{H} & 19 & \mathrm{r} 42 & 17 & \mathrm{a} 42 & 16 & \mathrm{~d} 42 \\ \mathrm{H} & 20 & \mathrm{r} 43 & 13 & \mathrm{a} 43 & 11 & \mathrm{~d} 43 \\ \mathrm{H} & 22 & \mathrm{r} 44 & 14 & \mathrm{a} 44 & 10 & \mathrm{~d} 44 \\ \mathrm{H} & 22 & \mathrm{r} 45 & 14 & \mathrm{a} 45 & 10 & \mathrm{~d} 45 \\ \mathrm{H} & 22 & \mathrm{r} 46 & 14 & \mathrm{a} 46 & 10 & \mathrm{~d} 46 \\ \mathrm{H} & 24 & \mathrm{r} 47 & 20 & \mathrm{a} 47 & 13 & \mathrm{~d} 47 \\ \mathrm{H} & 24 & \mathrm{r} 48 & 20 & \mathrm{a} 48 & 13 & \mathrm{~d} 48 \\ \mathrm{H} & 24 & \mathrm{r} 49 & 20 & \mathrm{a} 49 & 13 & \mathrm{~d} 49 \\ \text { Variables: } & & & & \end{array}$

ariables:

$\mathrm{r} 2=1.4002$

$\mathrm{r} 3=1.4113$

a3 $=120.87$

$\mathrm{r} 4=1.3898$

$\mathrm{a} 4=120.29$

$\mathrm{d} 4=359.57$

$\mathrm{r} 5=1.4068$

$\mathrm{a} 5=119.44$

$\mathrm{d} 5=0.32$

r6 $=1.3998$

a6 $=119.86$

$\mathrm{d} 6=359.90$

$\mathrm{r} 7=1.5272$

$\mathrm{a} 7=109.22$

$\mathrm{d} 7=181.14$

$\mathrm{r} 8=1.5652$

$\mathrm{a} 8=101.44$

$\mathrm{d} 8=348.92$

$\mathrm{r} 9=1.3849$

$\mathrm{a} 9=129.13$

$\mathrm{d} 9=181.69$ 


$$
\begin{aligned}
& \mathrm{r} 10=1.4804 \\
& \mathrm{a} 10=104.64 \\
& \mathrm{~d} 10=257.03 \\
& \mathrm{r} 11=1.4632 \\
& \mathrm{a} 11=113.11 \\
& \mathrm{~d} 11=\quad 3.01 \\
& \mathrm{r} 12=1.5390 \\
& \mathrm{a} 12=103.40 \\
& \mathrm{~d} 12=339.66 \\
& \mathrm{r} 13=1.5456 \\
& \mathrm{a} 13=116.13 \\
& \mathrm{~d} 13=102.84 \\
& \text { r14 }=1.3738 \\
& \mathrm{a} 14=118.65 \\
& \mathrm{~d} 14=194.98 \\
& \mathrm{r} 15=1.5538 \\
& \mathrm{a} 15=112.43 \\
& \mathrm{~d} 15=110.26 \\
& \mathrm{r} 16=1.5035 \\
& \text { a16 }=115.40 \\
& \mathrm{~d} 16=305.42 \\
& \text { r17 }=1.3475 \\
& \text { a17 }=128.15 \\
& \mathrm{~d} 17=238.38 \\
& \mathrm{r} 18=1.5102 \\
& \text { a18 }=120.63 \\
& \mathrm{~d} 18=180.77 \\
& \mathrm{r} 19=1.5089 \\
& \mathrm{a} 19=125.12 \\
& \mathrm{~d} 19=\quad 0.42 \\
& \mathrm{r} 20=1.3508 \\
& \mathrm{a} 20=116.71 \\
& \mathrm{~d} 20=\quad 5.37 \\
& \text { r21 }=1.2273 \\
& \mathrm{a} 21=118.97 \\
& \mathrm{~d} 21=183.32 \\
& \mathrm{r} 22=1.5159
\end{aligned}
$$


$\mathrm{a} 22=117.47$

$\mathrm{d} 22=171.42$

r23 $=1.2276$

$\mathrm{a} 23=120.32$

$\mathrm{d} 23=351.31$

r24= 1.4495

$\mathrm{a} 24=123.49$

$\mathrm{d} 24=180.40$

r25 $=1.1000$

$\mathrm{a} 25=110.86$

$\mathrm{d} 25=221.97$

r26 $=1.0988$

a26= 114.41

$\mathrm{d} 26=139.06$

r27 $=1.0926$

a27 $=120.91$

$\mathrm{d} 27=180.33$

r28 $=1.0941$

$\mathrm{a} 28=120.55$

$\mathrm{d} 28=180.06$

r29 $=1.0924$

a29 $=120.02$

$\mathrm{d} 29=179.71$

r30 $=1.0937$

$\mathrm{a} 30=119.61$

$\mathrm{d} 30=179.83$

r31 $=1.0132$

a31 $=122.82$

$\mathrm{d} 31=341.58$

r32 $=1.1005$

a32 $=109.06$

$\mathrm{d} 32=271.70$

r33 $=1.0978$

$\mathrm{a} 33=111.55$

$\mathrm{d} 33=152.72$

r34= 1.1062

$\mathrm{a} 34=106.58$ 


$$
\begin{aligned}
& \mathrm{d} 34=66.72 \\
& \text { r35 }=1.1004 \\
& \text { a35 }=108.00 \\
& \text { d } 35=180.44 \\
& \text { r36 }=1.0967 \\
& \text { a36 }=114.81 \\
& \mathrm{~d} 36=59.13 \\
& \text { r37 }=1.1049 \\
& \text { a } 37=110.98 \\
& \mathrm{~d} 37=120.90 \\
& \text { r38 }=1.1048 \\
& \mathrm{a} 38=110.91 \\
& \mathrm{~d} 38=238.64 \\
& \text { r39 }=1.1002 \\
& \text { a39 }=112.12 \\
& \mathrm{~d} 39=359.75 \\
& \mathrm{r} 40=1.1046 \\
& \mathrm{a} 40=110.46 \\
& \mathrm{~d} 40=239.79 \\
& \mathrm{r} 41=1.0979 \\
& \mathrm{a} 41=113.71 \\
& \mathrm{~d} 41=\quad 1.23 \\
& \mathrm{r} 42=1.1043 \\
& \mathrm{a} 42=110.44 \\
& \mathrm{~d} 42=122.72 \\
& \mathrm{r} 43=1.0137 \\
& \mathrm{a} 43=117.52 \\
& \mathrm{~d} 43=352.82 \\
& \text { r44 }=1.0966 \\
& \mathrm{a} 44=108.41 \\
& \mathrm{~d} 44=173.28 \\
& \mathrm{r} 45=1.1020 \\
& \mathrm{a} 45=110.67 \\
& \mathrm{~d} 45=292.63 \\
& \mathrm{r} 46=1.1001 \\
& \mathrm{a} 46=111.75 \\
& \mathrm{~d} 46=52.53
\end{aligned}
$$


$\mathrm{r} 47=1.1025$

$\mathrm{a} 47=111.31$

$\mathrm{d} 47=110.46$

$\mathrm{r} 48=1.1012$

$\mathrm{a} 48=110.83$

$\mathrm{d} 48=231.31$

$\mathrm{r} 49=1.0972$

$\mathrm{a} 49=108.26$

$\mathrm{d} 49=350.96$ 


\section{SRR12}
$\begin{array}{ll}0 & 1\end{array}$
C
C $\quad 1 \quad$ r2
C $2 \begin{array}{llll}\text { r } 3 & 1 & \text { a3 }\end{array}$
$\begin{array}{lllllll}\mathrm{C} & 3 & \mathrm{r} 4 & 2 & \mathrm{a} 4 & 1 & \mathrm{~d} 4\end{array}$
$\begin{array}{lllllll}\mathrm{C} & 4 & \mathrm{r} 5 & 3 & \mathrm{a} 5 & 2 & \mathrm{~d} 5\end{array}$
C $\quad 5 \quad$ r6 $64 \begin{array}{llll}\text { a6 } & 3 & \text { d6 }\end{array}$
$\begin{array}{lllllll}\mathrm{C} & 3 & \mathrm{r} 7 & 2 & \mathrm{a} 7 & 1 & \mathrm{~d} 7\end{array}$
$\begin{array}{lllllll}\mathrm{C} & 7 & \mathrm{r} 8 & 3 & \mathrm{a} 8 & 2 & \mathrm{~d} 8\end{array}$
$\begin{array}{lllllll}\mathrm{N} & 2 & \mathrm{r} 9 & 1 & \mathrm{a} 9 & 3 & \mathrm{~d} 9\end{array}$
$\begin{array}{lllllll}\mathrm{N} & 8 & \mathrm{r} 10 & 7 & \mathrm{a} 10 & 3 & \mathrm{~d} 10\end{array}$
$\begin{array}{lllllll}\text { C } & 10 & \text { r11 } & 8 & \text { a11 } & 7 & \text { d11 }\end{array}$
$\begin{array}{lllllll}\text { C } & 11 & \text { r12 } & 10 & \text { a12 } & 8 & \text { d12 }\end{array}$
$\begin{array}{lllllll}\text { C } & 11 & \text { r13 } & 10 & \text { a13 } & 8 & \text { d } 13\end{array}$
$\begin{array}{lllllll}\text { C } & 10 & \text { r14 } & 8 & \text { a14 } & 7 & \text { d14 }\end{array}$
$\begin{array}{lllllll}\text { C } & 7 & \text { r15 } & 3 & \text { a15 } & 2 & \text { d15 }\end{array}$
$\begin{array}{lllllll}\text { C } & 15 & \text { r16 } & 7 & \text { a16 } & 3 & \text { d } 16\end{array}$
$\begin{array}{lllllll}\mathrm{C} & 16 & \mathrm{r} 17 & 15 & \text { a17 } & 7 & \mathrm{~d} 17\end{array}$
$\begin{array}{lllllll}\text { C } & 17 & \text { r18 } & 16 & \text { a18 } & 15 & \text { d18 }\end{array}$
$\begin{array}{lllllll}\mathrm{C} & 17 & \mathrm{r} 19 & 16 & \mathrm{a} 19 & 15 & \mathrm{~d} 19\end{array}$
$\begin{array}{lllllll}\mathrm{N} & 13 & \mathrm{r} 20 & 11 & \mathrm{a} 20 & 10 & \mathrm{~d} 20\end{array}$
$\begin{array}{lllllll}\mathrm{O} & 13 & \mathrm{r} 21 & 11 & \mathrm{a} 21 & 10 & \mathrm{~d} 21\end{array}$
$\begin{array}{lllllll}\mathrm{C} & 14 & \mathrm{r} 22 & 10 & \mathrm{a} 22 & 8 & \mathrm{~d} 22\end{array}$
$\begin{array}{lllllll}\mathrm{O} & 14 & \mathrm{r} 23 & 10 & \text { a23 } & 8 & \mathrm{~d} 23\end{array}$
$\begin{array}{lllllll}\text { C } & 20 & \text { r24 } & 13 & \text { a24 } & 11 & \text { d } 24\end{array}$
$\begin{array}{lllllll}\mathrm{H} & 11 & \mathrm{r} 25 & 10 & \mathrm{a} 25 & 8 & \mathrm{~d} 25\end{array}$
$\begin{array}{lllllll}\mathrm{H} & 8 & \mathrm{r} 26 & 7 & \mathrm{a} 26 & 3 & \mathrm{~d} 26\end{array}$
$\begin{array}{lllllll}\mathrm{H} & 1 & \mathrm{r} 27 & 2 & \mathrm{a} 27 & 3 & \mathrm{~d} 27\end{array}$
$\begin{array}{lllllll}\mathrm{H} & 4 & \mathrm{r} 28 & 3 & \mathrm{a} 28 & 2 & \mathrm{~d} 28\end{array}$
$\begin{array}{lllllll}\mathrm{H} & 5 & \mathrm{r} 29 & 4 & \mathrm{a} 29 & 3 & \mathrm{~d} 29\end{array}$
$\begin{array}{lllllll}\mathrm{H} & 6 & \mathrm{r} 30 & 5 & \mathrm{a} 30 & 4 & \mathrm{~d} 30\end{array}$
$\begin{array}{lllllll}\mathrm{H} & 9 & \text { r31 } & 2 & \text { a31 } & 1 & \mathrm{~d} 31\end{array}$
$\begin{array}{lllllll}\mathrm{H} & 12 & \text { r32 } & 11 & \text { a32 } & 10 & \text { d } 32\end{array}$
$\begin{array}{lllllll}\mathrm{H} & 12 & \text { r33 } & 11 & \text { a33 } & 10 & \text { d } 33\end{array}$
$\begin{array}{lllllll}\mathrm{H} & 15 & \text { r34 } & 7 & \text { a34 } & 3 & \text { d34 }\end{array}$

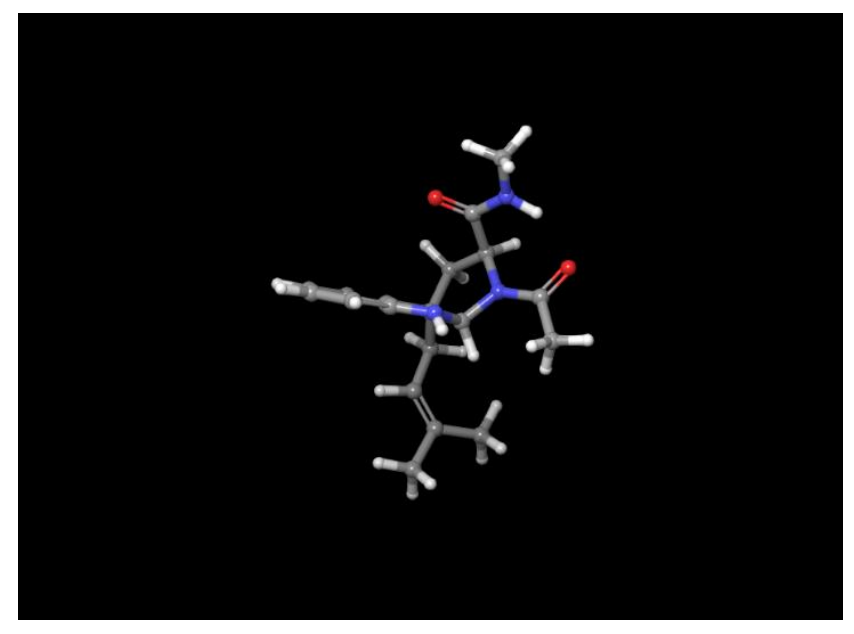




$$
\begin{aligned}
& \begin{array}{lllllll}
\mathrm{H} & 15 & \mathrm{r} 35 & 7 & \mathrm{a} 35 & 3 & \mathrm{~d} 35
\end{array} \\
& \begin{array}{lllllll}
\mathrm{H} & 16 & \mathrm{r} 36 & 15 & \mathrm{a} 36 & 7 & \mathrm{~d} 36
\end{array} \\
& \begin{array}{lllllll}
\mathrm{H} & 18 & \mathrm{r} 37 & 17 & \mathrm{a} 37 & 16 & \mathrm{~d} 37
\end{array} \\
& \begin{array}{lllllll}
\mathrm{H} & 18 & \mathrm{r} 38 & 17 & \mathrm{a} 38 & 16 & \mathrm{~d} 38
\end{array} \\
& \begin{array}{lllllll}
\mathrm{H} & 18 & \text { r39 } & 17 & \text { a39 } & 16 & \text { d } 39
\end{array} \\
& \begin{array}{lllllll}
\mathrm{H} & 19 & \mathrm{r} 40 & 17 & \mathrm{a} 40 & 16 & \mathrm{~d} 40
\end{array} \\
& \begin{array}{lllllll}
\mathrm{H} & 19 & \mathrm{r} 41 & 17 & \mathrm{a} 41 & 16 & \mathrm{~d} 41
\end{array} \\
& \begin{array}{lllllll}
\mathrm{H} & 19 & \mathrm{r} 42 & 17 & \mathrm{a} 42 & 16 & \mathrm{~d} 42
\end{array} \\
& \begin{array}{lllllll}
\mathrm{H} & 20 & \mathrm{r} 43 & 13 & \mathrm{a} 43 & 11 & \mathrm{~d} 43
\end{array} \\
& \begin{array}{lllllll}
\mathrm{H} & 22 & \mathrm{r} 44 & 14 & \mathrm{a} 44 & 10 & \mathrm{~d} 44
\end{array} \\
& \begin{array}{lllllll}
\mathrm{H} & 22 & \mathrm{r} 45 & 14 & \mathrm{a} 45 & 10 & \mathrm{~d} 45
\end{array} \\
& \begin{array}{lllllll}
\mathrm{H} & 22 & \mathrm{r} 46 & 14 & \mathrm{a} 46 & 10 & \mathrm{~d} 46
\end{array} \\
& \begin{array}{lllllll}
\mathrm{H} & 24 & \mathrm{r} 47 & 20 & \mathrm{a} 47 & 13 & \mathrm{~d} 47
\end{array} \\
& \begin{array}{lllllll}
\mathrm{H} & 24 & \mathrm{r} 48 & 20 & \mathrm{a} 48 & 13 & \mathrm{~d} 48
\end{array} \\
& \begin{array}{lllllll}
\mathrm{H} & 24 & \mathrm{r} 49 & 20 & \mathrm{a} 49 & 13 & \mathrm{~d} 49
\end{array} \\
& \text { Variables: } \\
& \mathrm{r} 2=1.3999 \\
& \text { r3 }=1.4075 \\
& \mathrm{a} 3=120.94 \\
& \mathrm{r} 4=1.3905 \\
& \mathrm{a} 4=120.21 \\
& \mathrm{~d} 4=\quad 1.07 \\
& \mathrm{r} 5=1.4061 \\
& \mathrm{a} 5=119.49 \\
& \mathrm{~d} 5=359.20 \\
& \text { r6= } 1.3999 \\
& \mathrm{a} 6=119.88 \\
& \mathrm{~d} 6=0.01 \\
& \mathrm{r} 7=1.5209 \\
& \mathrm{a} 7=109.57 \\
& \mathrm{~d} 7=181.20 \\
& \mathrm{r} 8=1.5734 \\
& \mathrm{a} 8=102.57 \\
& \mathrm{~d} 8=\quad 3.24 \\
& \mathrm{r} 9=1.3897 \\
& \mathrm{a} 9=128.28 \\
& \mathrm{~d} 9=178.65
\end{aligned}
$$




$$
\begin{aligned}
& \mathrm{r} 10=1.4612 \\
& \mathrm{a} 10=105.18 \\
& \mathrm{~d} 10=232.25 \\
& \mathrm{r} 11=1.4704 \\
& \mathrm{a} 11=111.65 \\
& \mathrm{~d} 11=24.06 \\
& \mathrm{r} 12=1.5333 \\
& \mathrm{a} 12=103.94 \\
& \mathrm{~d} 12=331.58 \\
& \mathrm{r} 13=1.5527 \\
& \mathrm{a} 13=112.61 \\
& \mathrm{~d} 13=94.85 \\
& \mathrm{r} 14=1.3620 \\
& \mathrm{a} 14=126.66 \\
& \mathrm{~d} 14=204.30 \\
& \mathrm{r} 15=1.5538 \\
& \mathrm{a} 15=113.36 \\
& \mathrm{~d} 15=124.43 \\
& \mathrm{r} 16=1.5034 \\
& \mathrm{a} 16=115.12 \\
& \mathrm{~d} 16=305.76 \\
& \mathrm{r} 17=1.3482 \\
& \mathrm{a} 17=128.18 \\
& \mathrm{~d} 17=241.63 \\
& \mathrm{r} 18=1.5100 \\
& \mathrm{a} 18=120.66 \\
& \mathrm{~d} 18=180.54 \\
& \mathrm{r} 19=1.5090 \\
& \mathrm{a} 19=125.06 \\
& \mathrm{~d} 19=
\end{aligned}
$$


$\mathrm{a} 22=117.91$

$\mathrm{d} 22=\quad 5.87$

r23 $=1.2353$

$\mathrm{a} 23=121.00$

$\mathrm{d} 23=185.63$

r24 $=1.4480$

$\mathrm{a} 24=123.61$

$\mathrm{d} 24=181.55$

r25 $=1.1004$

$\mathrm{a} 25=107.64$

$\mathrm{d} 25=212.40$

r26 $=1.0988$

a26 $=112.25$

$\mathrm{d} 26=113.55$

r27 $=1.0927$

a27 $=120.88$

$\mathrm{d} 27=179.61$

r28 $=1.0941$

a28 $=120.50$

$\mathrm{d} 28=179.80$

r29 $=1.0926$

$\mathrm{a} 29=120.01$

$\mathrm{d} 29=180.38$

r30 $=1.0935$

$\mathrm{a} 30=119.68$

$\mathrm{d} 30=180.43$

r31 $=1.0142$

a31 $=119.28$

$\mathrm{d} 31=29.87$

r32 $=1.0994$

a32 $=109.41$

$\mathrm{d} 32=261.64$

r33 $=1.0962$

a33 $=110.36$

$\mathrm{d} 33=142.52$

r34= 1.1059

a34= 106.85 


$$
\begin{aligned}
& \mathrm{d} 34=67.30 \\
& \text { r35 }=1.1006 \\
& \text { a35 }=107.90 \\
& \text { d } 35=180.88 \\
& \text { r36 }=1.0967 \\
& \text { a36 }=114.83 \\
& \text { d } 36=61.88 \\
& \text { r37 }=1.1049 \\
& \text { a } 37=110.90 \\
& \mathrm{~d} 37=120.31 \\
& \text { r38 }=1.1046 \\
& \text { a38 }=110.95 \\
& \mathrm{~d} 38=238.01 \\
& \text { r39 }=1.1000 \\
& \text { a39 }=112.14 \\
& \mathrm{~d} 39=359.21 \\
& \mathrm{r} 40=1.1045 \\
& \mathrm{a} 40=110.42 \\
& \mathrm{~d} 40=239.17 \\
& \mathrm{r} 41=1.0979 \\
& \mathrm{a} 41=113.74 \\
& \mathrm{~d} 41=0.58 \\
& \mathrm{r} 42=1.1044 \\
& \mathrm{a} 42=110.44 \\
& \mathrm{~d} 42=122.14 \\
& \mathrm{r} 43=1.0221 \\
& \mathrm{a} 43=116.67 \\
& \mathrm{~d} 43=358.09 \\
& \mathrm{r} 44=1.0965 \\
& \text { a44= } 108.02 \\
& \mathrm{~d} 44=178.10 \\
& \mathrm{r} 45=1.1006 \\
& \mathrm{a} 45=111.40 \\
& \mathrm{~d} 45=297.51 \\
& \mathrm{r} 46=1.1008 \\
& \mathrm{a} 46=111.64 \\
& \mathrm{~d} 46=58.02
\end{aligned}
$$


$\mathrm{r} 47=1.0973$

$\mathrm{a} 47=108.58$

$\mathrm{d} 47=356.01$

$\mathrm{r} 48=1.1032$

$\mathrm{a} 48=111.45$

$\mathrm{d} 48=115.72$

$\mathrm{r} 49=1.1025$

$\mathrm{a} 49=111.11$

$\mathrm{d} 49=236.40$ 


\section{SRR13}
$\begin{array}{ll}0 & 1\end{array}$
C
C 11 r2
C $2 \begin{array}{llll}\text { r } 3 & 1 & \text { a3 }\end{array}$
$\begin{array}{lllllll}\mathrm{C} & 3 & \mathrm{r} 4 & 2 & \mathrm{a} 4 & 1 & \mathrm{~d} 4\end{array}$
C $\quad 4 \quad$ r5 3 a5 2 d5

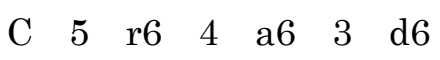
$\begin{array}{lllllll}\mathrm{C} & 3 & \mathrm{r} 7 & 2 & \text { a7 } & 1 & \mathrm{~d} 7\end{array}$
$\begin{array}{lllllll}\mathrm{C} & 7 & \mathrm{r} 8 & 3 & \mathrm{a} 8 & 2 & \mathrm{~d} 8\end{array}$
$\begin{array}{lllllll}\mathrm{N} & 2 & \mathrm{r} 9 & 1 & \mathrm{a} 9 & 3 & \mathrm{~d} 9\end{array}$
$\begin{array}{lllllll}\mathrm{N} & 8 & \mathrm{r} 10 & 7 & \mathrm{a} 10 & 3 & \mathrm{~d} 10\end{array}$
$\begin{array}{lllllll}\text { C } & 10 & \text { r11 } & 8 & \text { a11 } & 7 & \text { d11 }\end{array}$
$\begin{array}{lllllll}\text { C } & 11 & \text { r12 } & 10 & \text { a } 12 & 8 & \text { d12 }\end{array}$
$\begin{array}{lllllll}\text { C } & 11 & \text { r13 } & 10 & \text { a13 } & 8 & \text { d13 }\end{array}$
$\begin{array}{lllllll}\text { C } & 10 & \text { r14 } & 8 & \text { a14 } & 7 & \text { d14 }\end{array}$
$\begin{array}{lllllll}\text { C } & 7 & \text { r15 } & 3 & \text { a15 } & 2 & \text { d15 }\end{array}$
$\begin{array}{lllllll}\text { C } & 15 & \text { r16 } & 7 & \text { a16 } & 3 & \text { d } 16\end{array}$
$\begin{array}{lllllll}\mathrm{C} & 16 & \mathrm{r} 17 & 15 & \text { a17 } & 7 & \mathrm{~d} 17\end{array}$
$\begin{array}{lllllll}\text { C } & 17 & \text { r18 } & 16 & \text { a18 } & 15 & \text { d18 }\end{array}$
$\begin{array}{lllllll}\mathrm{C} & 17 & \mathrm{r} 19 & 16 & \mathrm{a} 19 & 15 & \mathrm{~d} 19\end{array}$
$\begin{array}{lllllll}\mathrm{N} & 13 & \mathrm{r} 20 & 11 & \mathrm{a} 20 & 10 & \mathrm{~d} 20\end{array}$
$\begin{array}{lllllll}\mathrm{O} & 13 & \mathrm{r} 21 & 11 & \mathrm{a} 21 & 10 & \mathrm{~d} 21\end{array}$
$\begin{array}{lllllll}\mathrm{C} & 14 & \mathrm{r} 22 & 10 & \mathrm{a} 22 & 8 & \mathrm{~d} 22\end{array}$
$\begin{array}{lllllll}\mathrm{O} & 14 & \mathrm{r} 23 & 10 & \mathrm{a} 23 & 8 & \mathrm{~d} 23\end{array}$
$\begin{array}{lllllll}\text { C } & 20 & \text { r24 } & 13 & \text { a24 } & 11 & \text { d } 24\end{array}$
$\begin{array}{lllllll}\mathrm{H} & 11 & \mathrm{r} 25 & 10 & \mathrm{a} 25 & 8 & \mathrm{~d} 25\end{array}$
$\begin{array}{lllllll}\mathrm{H} & 8 & \mathrm{r} 26 & 7 & \mathrm{a} 26 & 3 & \mathrm{~d} 26\end{array}$
$\begin{array}{lllllll}\mathrm{H} & 1 & \mathrm{r} 27 & 2 & \mathrm{a} 27 & 3 & \mathrm{~d} 27\end{array}$
$\begin{array}{lllllll}\mathrm{H} & 4 & \mathrm{r} 28 & 3 & \mathrm{a} 28 & 2 & \mathrm{~d} 28\end{array}$
$\begin{array}{lllllll}\mathrm{H} & 5 & \mathrm{r} 29 & 4 & \mathrm{a} 29 & 3 & \mathrm{~d} 29\end{array}$
$\begin{array}{lllllll}\mathrm{H} & 6 & \mathrm{r} 30 & 5 & \mathrm{a} 30 & 4 & \mathrm{~d} 30\end{array}$
$\begin{array}{lllllll}\mathrm{H} & 9 & \text { r31 } & 2 & \text { a31 } & 1 & \text { d } 31\end{array}$
$\begin{array}{lllllll}\mathrm{H} & 12 & \text { r32 } & 11 & \text { a32 } & 10 & \text { d } 32\end{array}$
$\begin{array}{lllllll}\mathrm{H} & 12 & \text { r33 } & 11 & \text { a33 } & 10 & \text { d } 33\end{array}$
$\begin{array}{lllllll}\mathrm{H} & 15 & \text { r34 } & 7 & \text { a34 } & 3 & \text { d34 }\end{array}$

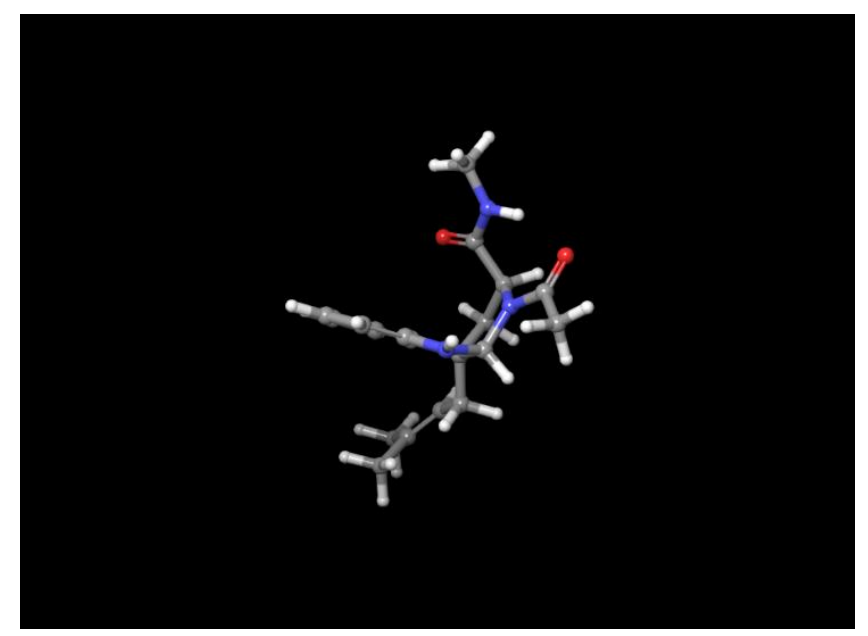




$\begin{array}{lllllll}\mathrm{H} & 15 & \mathrm{r} 35 & 7 & \mathrm{a} 35 & 3 & \mathrm{~d} 35 \\ \mathrm{H} & 16 & \mathrm{r} 36 & 15 & \mathrm{a} 36 & 7 & \mathrm{~d} 36 \\ \mathrm{H} & 18 & \mathrm{r} 37 & 17 & \mathrm{a} 37 & 16 & \mathrm{~d} 37 \\ \mathrm{H} & 18 & \mathrm{r} 38 & 17 & \mathrm{a} 38 & 16 & \mathrm{~d} 38 \\ \mathrm{H} & 18 & \mathrm{r} 39 & 17 & \mathrm{a} 39 & 16 & \mathrm{~d} 39 \\ \mathrm{H} & 19 & \mathrm{r} 40 & 17 & \mathrm{a} 40 & 16 & \mathrm{~d} 40 \\ \mathrm{H} & 19 & \mathrm{r} 41 & 17 & \mathrm{a} 41 & 16 & \mathrm{~d} 41 \\ \mathrm{H} & 19 & \mathrm{r} 42 & 17 & \mathrm{a} 42 & 16 & \mathrm{~d} 42 \\ \mathrm{H} & 20 & \mathrm{r} 43 & 13 & \mathrm{a} 43 & 11 & \mathrm{~d} 43 \\ \mathrm{H} & 22 & \mathrm{r} 44 & 14 & \mathrm{a} 44 & 10 & \mathrm{~d} 44 \\ \mathrm{H} & 22 & \mathrm{r} 45 & 14 & \mathrm{a} 45 & 10 & \mathrm{~d} 45 \\ \mathrm{H} & 22 & \mathrm{r} 46 & 14 & \mathrm{a} 46 & 10 & \mathrm{~d} 46 \\ \mathrm{H} & 24 & \mathrm{r} 47 & 20 & \mathrm{a} 47 & 13 & \mathrm{~d} 47 \\ \mathrm{H} & 24 & \mathrm{r} 48 & 20 & \mathrm{a} 48 & 13 & \mathrm{~d} 48 \\ \mathrm{H} & 24 & \mathrm{r} 49 & 20 & \mathrm{a} 49 & 13 & \mathrm{~d} 49 \\ \text { Variables: } & & & & \end{array}$

$\mathrm{r} 2=1.3995$

$\mathrm{r} 3=1.4086$

a3 $=121.25$

$\mathrm{r} 4=1.3902$

$\mathrm{a} 4=119.99$

$\mathrm{d} 4=359.72$

r5 $=1.4059$

$\mathrm{a} 5=119.48$

$\mathrm{d} 5=0.04$

r6 $=1.3999$

$\mathrm{a} 6=120.03$

$\mathrm{d} 6=0.11$

$\mathrm{r} 7=1.5270$

$\mathrm{a} 7=109.07$

$\mathrm{d} 7=181.62$

$\mathrm{r} 8=1.5685$

$\mathrm{a} 8=101.48$

d $8=347.84$

$\mathrm{r} 9=1.3888$

$\mathrm{a} 9=128.70$

$\mathrm{d} 9=182.13$ 


$$
\begin{aligned}
& \mathrm{r} 10=1.4810 \\
& \mathrm{a} 10=104.55 \\
& \mathrm{~d} 10=257.56 \\
& \mathrm{r} 11=1.4770 \\
& \mathrm{a} 11=113.03 \\
& \mathrm{~d} 11=0.24 \\
& \mathrm{r} 12=1.5303 \\
& \mathrm{a} 12=103.65 \\
& \mathrm{~d} 12=342.48 \\
& \mathrm{r} 13=1.5528 \\
& \mathrm{a} 13=115.05 \\
& \mathrm{~d} 13=107.00 \\
& \mathrm{r} 14=1.3634 \\
& \mathrm{a} 14=124.74 \\
& \mathrm{~d} 14=193.56 \\
& \mathrm{r} 15=1.5556 \\
& \mathrm{a} 15=111.67 \\
& \mathrm{~d} 15=105.77 \\
& \mathrm{r} 16=1.5031 \\
& \mathrm{a} 16=114.77 \\
& \mathrm{~d} 16=70.25 \\
& \mathrm{r} 17=1.3474 \\
& \mathrm{a} 17=128.37 \\
& \mathrm{~d} 17=239.17 \\
& \mathrm{r} 18=1.5098 \\
& \mathrm{a} 18=120.70 \\
& \mathrm{~d} 18=181.13 \\
& \mathrm{r} 19=1.5080 \\
& \mathrm{a} 19=124.92 \\
& \mathrm{~d} 19=
\end{aligned}
$$


$\mathrm{a} 22=118.12$

$\mathrm{d} 22=359.20$

r23 $=1.2355$

a23 $=121.07$

$\mathrm{d} 23=179.02$

r24= 1.4481

a24= 123.38

$\mathrm{d} 24=177.64$

r25 $=1.1023$

$\mathrm{a} 25=107.55$

$\mathrm{d} 25=224.26$

r26 $=1.0994$

$\mathrm{a} 26=112.80$

$\mathrm{d} 26=139.53$

r27 $=1.0928$

a27 $=120.96$

$\mathrm{d} 27=180.45$

r28 $=1.0921$

$\mathrm{a} 28=120.42$

$\mathrm{d} 28=179.46$

r29 $=1.0927$

a29 $=119.94$

$\mathrm{d} 29=179.68$

r30 $=1.0937$

$\mathrm{a} 30=119.69$

$\mathrm{d} 30=179.63$

r31 $=1.0128$

a31 $=120.87$

$\mathrm{d} 31=340.90$

r32 $=1.1007$

a32 $=109.20$

$\mathrm{d} 32=269.78$

r33 $=1.0963$

$\mathrm{a} 33=110.79$

$\mathrm{d} 33=150.33$

r34= 1.1067

$\mathrm{a} 34=106.83$ 


$$
\begin{aligned}
& \mathrm{d} 34=190.74 \\
& \mathrm{r} 35=1.0999 \\
& \mathrm{a} 35=108.31 \\
& \mathrm{~d} 35=305.10 \\
& \mathrm{r} 36=1.0975 \\
& \mathrm{a} 36=114.80 \\
& \mathrm{~d} 36=60.34 \\
& \mathrm{r} 37=1.1048 \\
& \mathrm{a} 37=110.96 \\
& \mathrm{~d} 37=120.99 \\
& \mathrm{r} 38=1.1048 \\
& \mathrm{a} 38=110.90 \\
& \mathrm{~d} 38=238.71 \\
& \mathrm{r} 39=1.1002 \\
& \mathrm{a} 39=112.15 \\
& \mathrm{~d} 39=359.84 \\
& \mathrm{r} 40=1.1045 \\
& \mathrm{a} 40=110.50 \\
& \mathrm{~d} 40=238.77 \\
& \mathrm{~d} 46=116=56.93 \\
& \mathrm{r} 41=1.0978 \\
& \mathrm{a} 41=113.60 \\
& \mathrm{~d} 41=0.32 \\
& \mathrm{r} 42=1.1044 \\
& \mathrm{a} 42=110.37 \\
& \mathrm{~d} 42=121.63 \\
& \mathrm{r} 43=1.0229 \\
& \mathrm{a} 43=116.59 \\
& \mathrm{~d} 43= \\
& \mathrm{r} 44=1.0965 \\
& \mathrm{a} 44=108.00 \\
& \mathrm{~d} 44=177.15 \\
& \mathrm{~d} 45
\end{aligned}
$$


$\mathrm{r} 47=1.1020$

$\mathrm{a} 47=110.90$

$\mathrm{d} 47=129.24$

$\mathrm{r} 48=1.1038$

$\mathrm{a} 48=111.62$

$\mathrm{d} 48=249.87$

$\mathrm{r} 49=1.0973$

$\mathrm{a} 49=108.69$

$\mathrm{d} 49=9.46$ 


\section{SRR14}
$\begin{array}{ll}0 & 1\end{array}$
C
C 11 r2
C $2 \begin{array}{llll}\text { r } 3 & 1 & \text { a3 }\end{array}$
$\begin{array}{lllllll}\mathrm{C} & 3 & \mathrm{r} 4 & 2 & \mathrm{a} 4 & 1 & \mathrm{~d} 4\end{array}$
C $\quad 4 \quad$ r5 $\quad 3 \quad$ a5 $\quad 2$ d5

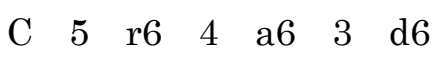
$\begin{array}{lllllll}\mathrm{C} & 3 & \mathrm{r} 7 & 2 & \mathrm{a} 7 & 1 & \mathrm{~d} 7\end{array}$
$\begin{array}{lllllll}\mathrm{C} & 7 & \mathrm{r} 8 & 3 & \mathrm{a} 8 & 2 & \mathrm{~d} 8\end{array}$
$\begin{array}{lllllll}\mathrm{N} & 2 & \mathrm{r} 9 & 1 & \mathrm{a} 9 & 3 & \mathrm{~d} 9\end{array}$
$\begin{array}{lllllll}\mathrm{N} & 8 & \mathrm{r} 10 & 7 & \mathrm{a} 10 & 3 & \mathrm{~d} 10\end{array}$
$\begin{array}{lllllll}\text { C } & 10 & \text { r11 } & 8 & \text { a11 } & 7 & \text { d11 }\end{array}$
$\begin{array}{lllllll}\text { C } & 11 & \text { r12 } & 10 & \text { a12 } & 8 & \text { d12 }\end{array}$
$\begin{array}{lllllll}\text { C } & 11 & \text { r13 } & 10 & \text { a13 } & 8 & \text { d13 }\end{array}$
$\begin{array}{lllllll}\text { C } & 10 & \text { r14 } & 8 & \text { a14 } & 7 & \text { d14 }\end{array}$
$\begin{array}{lllllll}\text { C } & 7 & \text { r15 } & 3 & \text { a15 } & 2 & \text { d } 15\end{array}$
$\begin{array}{lllllll}\text { C } & 15 & \text { r16 } & 7 & \text { a16 } & 3 & \text { d } 16\end{array}$
$\begin{array}{lllllll}\text { C } & 16 & \text { r17 } & 15 & \text { a17 } & 7 & \text { d17 }\end{array}$
$\begin{array}{lllllll}\text { C } & 17 & \text { r18 } & 16 & \text { a18 } & 15 & \text { d18 }\end{array}$
$\begin{array}{lllllll}\text { C } & 17 & \text { r19 } & 16 & \text { a } 19 & 15 & \text { d } 19\end{array}$
$\begin{array}{lllllll}\mathrm{N} & 13 & \mathrm{r} 20 & 11 & \mathrm{a} 20 & 10 & \mathrm{~d} 20\end{array}$
$\begin{array}{lllllll}\mathrm{O} & 13 & \mathrm{r} 21 & 11 & \mathrm{a} 21 & 10 & \mathrm{~d} 21\end{array}$
$\begin{array}{lllllll}\mathrm{C} & 14 & \mathrm{r} 22 & 10 & \mathrm{a} 22 & 8 & \mathrm{~d} 22\end{array}$
$\begin{array}{lllllll}\mathrm{O} & 14 & \mathrm{r} 23 & 10 & \mathrm{a} 23 & 8 & \mathrm{~d} 23\end{array}$
$\begin{array}{lllllll}\text { C } & 20 & \text { r24 } & 13 & \text { a24 } & 11 & \text { d } 24\end{array}$
$\begin{array}{lllllll}\mathrm{H} & 11 & \mathrm{r} 25 & 10 & \mathrm{a} 25 & 8 & \mathrm{~d} 25\end{array}$
$\begin{array}{lllllll}\mathrm{H} & 8 & \mathrm{r} 26 & 7 & \mathrm{a} 26 & 3 & \mathrm{~d} 26\end{array}$
$\begin{array}{lllllll}\mathrm{H} & 1 & \mathrm{r} 27 & 2 & \mathrm{a} 27 & 3 & \mathrm{~d} 27\end{array}$
$\begin{array}{lllllll}\mathrm{H} & 4 & \mathrm{r} 28 & 3 & \mathrm{a} 28 & 2 & \mathrm{~d} 28\end{array}$
$\begin{array}{lllllll}\mathrm{H} & 5 & \mathrm{r} 29 & 4 & \mathrm{a} 29 & 3 & \mathrm{~d} 29\end{array}$
$\begin{array}{lllllll}\mathrm{H} & 6 & \mathrm{r} 30 & 5 & \mathrm{a} 30 & 4 & \mathrm{~d} 30\end{array}$
$\begin{array}{lllllll}\mathrm{H} & 9 & \mathrm{r} 31 & 2 & \mathrm{a} 31 & 1 & \mathrm{~d} 31\end{array}$
$\begin{array}{lllllll}\mathrm{H} & 12 & \text { r32 } & 11 & \text { a32 } & 10 & \text { d } 32\end{array}$
$\begin{array}{lllllll}\mathrm{H} & 12 & \text { r33 } & 11 & \mathrm{a} 33 & 10 & \mathrm{~d} 33\end{array}$
$\begin{array}{lllllll}\mathrm{H} & 15 & \mathrm{r} 34 & 7 & \mathrm{a} 34 & 3 & \mathrm{~d} 34\end{array}$






$\begin{array}{lllllll}\mathrm{H} & 15 & \mathrm{r} 35 & 7 & \mathrm{a} 35 & 3 & \mathrm{~d} 35 \\ \mathrm{H} & 16 & \mathrm{r} 36 & 15 & \mathrm{a} 36 & 7 & \mathrm{~d} 36 \\ \mathrm{H} & 18 & \mathrm{r} 37 & 17 & \mathrm{a} 37 & 16 & \mathrm{~d} 37 \\ \mathrm{H} & 18 & \mathrm{r} 38 & 17 & \mathrm{a} 38 & 16 & \mathrm{~d} 38 \\ \mathrm{H} & 18 & \mathrm{r} 39 & 17 & \mathrm{a} 39 & 16 & \mathrm{~d} 39 \\ \mathrm{H} & 19 & \mathrm{r} 40 & 17 & \mathrm{a} 40 & 16 & \mathrm{~d} 40 \\ \mathrm{H} & 19 & \mathrm{r} 41 & 17 & \mathrm{a} 41 & 16 & \mathrm{~d} 41 \\ \mathrm{H} & 19 & \mathrm{r} 42 & 17 & \mathrm{a} 42 & 16 & \mathrm{~d} 42 \\ \mathrm{H} & 20 & \mathrm{r} 43 & 13 & \mathrm{a} 43 & 11 & \mathrm{~d} 43 \\ \mathrm{H} & 22 & \mathrm{r} 44 & 14 & \mathrm{a} 44 & 10 & \mathrm{~d} 44 \\ \mathrm{H} & 22 & \mathrm{r} 45 & 14 & \mathrm{a} 45 & 10 & \mathrm{~d} 45 \\ \mathrm{H} & 22 & \mathrm{r} 46 & 14 & \mathrm{a} 46 & 10 & \mathrm{~d} 46 \\ \mathrm{H} & 24 & \mathrm{r} 47 & 20 & \mathrm{a} 47 & 13 & \mathrm{~d} 47 \\ \mathrm{H} & 24 & \mathrm{r} 48 & 20 & \mathrm{a} 48 & 13 & \mathrm{~d} 48 \\ \mathrm{H} & 24 & \mathrm{r} 49 & 20 & \mathrm{a} 49 & 13 & \mathrm{~d} 49 \\ \text { Variables: } & & & & \end{array}$

$\mathrm{r} 2=1.3993$

$\mathrm{r} 3=1.4079$

a3 $=121.27$

$\mathrm{r} 4=1.3927$

$\mathrm{a} 4=119.79$

$\mathrm{d} 4=2.28$

r5 $=1.4045$

$\mathrm{a} 5=119.55$

$\mathrm{d} 5=358.24$

r6 $=1.4004$

$\mathrm{a} 6=120.10$

$\mathrm{d} 6=359.98$

$\mathrm{r} 7=1.5275$

$a 7=109.38$

$\mathrm{d} 7=185.18$

$\mathrm{r} 8=1.5695$

$\mathrm{a} 8=101.28$

$\mathrm{d} 8=347.70$

$\mathrm{r} 9=1.3961$

$\mathrm{a} 9=127.74$

$\mathrm{d} 9=177.80$ 


$$
\begin{aligned}
& \mathrm{r} 10=1.4777 \\
& \mathrm{a} 10=104.52 \\
& \mathrm{~d} 10=252.65 \\
& \mathrm{r} 11=1.4747 \\
& \mathrm{a} 11=113.24 \\
& \mathrm{~d} 11= \\
& \mathrm{r} 12=1.5301 \\
& \mathrm{a} 12=103.71 \\
& \mathrm{~d} 12=340.75 \\
& \mathrm{r} 13=1.5548 \\
& \mathrm{a} 13=114.35 \\
& \mathrm{~d} 13=104.54 \\
& \mathrm{r} 14=1.3609 \\
& \mathrm{a} 14=124.80 \\
& \mathrm{~d} 14=191.42 \\
& \mathrm{r} 15=1.5590 \\
& \mathrm{a} 15=111.74 \\
& \mathrm{~d} 15=104.87 \\
& \mathrm{r} 16=1.5035 \\
& \mathrm{a} 16=115.46 \\
& \mathrm{~d} 16=68.03 \\
& \mathrm{r} 17=1.3480 \\
& \mathrm{a} 17=128.18 \\
& \mathrm{~d} 17=114.00 \\
& \mathrm{r} 18=1.5101 \\
& \mathrm{a} 18=120.62 \\
& \mathrm{~d} 18=179.20 \\
& \mathrm{r} 19=1.5087 \\
& \mathrm{a} 19=125.14 \\
& \mathrm{~d} 19=359.57 \\
& \mathrm{r} 20=1.3512 \\
& \mathrm{~d} 20.51
\end{aligned}
$$




$$
\begin{aligned}
& \mathrm{a} 22=117.65 \\
& \mathrm{~d} 22=359.14 \\
& \mathrm{r} 23=1.2367 \\
& \mathrm{a} 23=121.31 \\
& \mathrm{~d} 23=179.78 \\
& \text { r24 }=1.4480 \\
& \text { a24= } 123.49 \\
& \mathrm{~d} 24=179.21 \\
& \mathrm{r} 25=1.1018 \\
& \text { a25 }=107.77 \\
& \mathrm{~d} 25=222.15 \\
& \text { r26 }=1.0987 \\
& \mathrm{a} 26=112.60 \\
& \mathrm{~d} 26=134.39 \\
& \text { r27 }=1.0925 \\
& \mathrm{a} 27=120.73 \\
& \mathrm{~d} 27=179.80 \\
& \text { r28 }=1.0923 \\
& \mathrm{a} 28=120.56 \\
& \mathrm{~d} 28=178.31 \\
& \text { r29 }=1.0926 \\
& \mathrm{a} 29=119.88 \\
& \mathrm{~d} 29=180.10 \\
& \text { r30 }=1.0935 \\
& \mathrm{a} 30=119.73 \\
& \mathrm{~d} 30=180.41 \\
& \text { r31 }=1.0139 \\
& \mathrm{a} 31=119.28 \\
& \mathrm{~d} 31=41.72 \\
& \text { r32 }=1.0998 \\
& \text { a32 }=109.30 \\
& \mathrm{~d} 32=268.59 \\
& \text { r33 }=1.0962 \\
& \mathrm{a} 33=110.87 \\
& \mathrm{~d} 33=149.29 \\
& \text { r34= } 1.1009 \\
& \text { a34 }=107.47
\end{aligned}
$$




$$
\begin{aligned}
& \mathrm{d} 34=192.22 \\
& \text { r35 }=1.1051 \\
& \text { a } 35=107.31 \\
& \mathrm{~d} 35=306.36 \\
& \text { r36 }=1.0959 \\
& \mathrm{a} 36=115.04 \\
& \mathrm{~d} 36=292.89 \\
& \text { r37 }=1.1047 \\
& \text { a } 37=110.92 \\
& \mathrm{~d} 37=121.78 \\
& \text { r38 }=1.1049 \\
& \text { a38 }=110.94 \\
& \mathrm{~d} 38=239.49 \\
& \text { r39 }=1.1001 \\
& \text { a39 }=112.15 \\
& \mathrm{~d} 39=0.64 \\
& \mathrm{r} 40=1.1043 \\
& \mathrm{a} 40=110.44 \\
& \mathrm{~d} 40=239.59 \\
& \mathrm{r} 41=1.0979 \\
& \mathrm{a} 41=113.72 \\
& \mathrm{~d} 41=\quad 1.03 \\
& \mathrm{r} 42=1.1044 \\
& \mathrm{a} 42=110.42 \\
& \mathrm{~d} 42=122.52 \\
& \mathrm{r} 43=1.0231 \\
& \mathrm{a} 43=116.63 \\
& \mathrm{~d} 43=1.35 \\
& \text { r44 }=1.0965 \\
& \text { a44= } 108.32 \\
& \mathrm{~d} 44=178.08 \\
& \mathrm{r} 45=1.1002 \\
& \mathrm{a} 45=110.39 \\
& \mathrm{~d} 45=297.64 \\
& \mathrm{r} 46=1.1007 \\
& \mathrm{a} 46=111.88 \\
& \mathrm{~d} 46=57.52
\end{aligned}
$$


$\mathrm{r} 47=1.0974$

$\mathrm{a} 47=108.68$

$\mathrm{d} 47=\quad 5.96$

$\mathrm{r} 48=1.1022$

$\mathrm{a} 48=111.01$

$\mathrm{d} 48=125.72$

$\mathrm{r} 49=1.1034$

$\mathrm{a} 49=111.52$

$\mathrm{d} 49=246.33$ 


\section{SRR19}
$\begin{array}{ll}0 & 1\end{array}$
C
C 11 r2
C $2 \begin{array}{llll}\text { r } 3 & 1 & \text { a3 }\end{array}$
$\begin{array}{lllllll}\text { C } & 3 & \text { r4 } & 2 & \text { a } 4 & 1 & \mathrm{~d} 4\end{array}$
$\begin{array}{lllllll}\mathrm{C} & 4 & \mathrm{r} 5 & 3 & \mathrm{a} 5 & 2 & \mathrm{~d} 5\end{array}$
$\begin{array}{lllllll}\mathrm{C} & 5 & \mathrm{r} 6 & 4 & \mathrm{a} 6 & 3 & \mathrm{~d} 6\end{array}$
$\begin{array}{lllllll}\mathrm{C} & 3 & \mathrm{r} 7 & 2 & \mathrm{a} 7 & 1 & \mathrm{~d} 7\end{array}$
$\begin{array}{lllllll}\mathrm{C} & 7 & \mathrm{r} 8 & 3 & \mathrm{a} 8 & 2 & \mathrm{~d} 8\end{array}$
$\begin{array}{lllllll}\mathrm{N} & 2 & \mathrm{r} 9 & 1 & \mathrm{a} 9 & 3 & \mathrm{~d} 9\end{array}$
$\begin{array}{lllllll}\mathrm{N} & 8 & \mathrm{r} 10 & 7 & \mathrm{a} 10 & 3 & \mathrm{~d} 10\end{array}$
$\begin{array}{lllllll}\text { C } & 10 & \text { r11 } & 8 & \text { a11 } & 7 & \text { d11 }\end{array}$
$\begin{array}{lllllll}\text { C } & 7 & \text { r12 } & 3 & \text { a12 } & 2 & \text { d12 }\end{array}$
$\begin{array}{lllllll}\text { C } & 11 & \text { r13 } & 10 & \text { a13 } & 8 & \text { d } 13\end{array}$
$\begin{array}{lllllll}\text { C } & 10 & \text { r14 } & 8 & \text { a14 } & 7 & \text { d14 }\end{array}$
$\begin{array}{lllllll}\text { C } & 7 & \text { r15 } & 3 & \text { a15 } & 2 & \text { d } 15\end{array}$
$\begin{array}{lllllll}\text { C } & 15 & \text { r16 } & 7 & \text { a16 } & 3 & \text { d16 }\end{array}$
$\begin{array}{lllllll}\text { C } & 16 & \text { r17 } & 15 & \text { a17 } & 7 & \text { d } 17\end{array}$
$\begin{array}{lllllll}\text { C } & 17 & \text { r18 } & 16 & \text { a18 } & 15 & \text { d18 }\end{array}$
$\begin{array}{lllllll}\text { C } & 17 & \text { r19 } & 16 & \text { a19 } & 15 & \text { d19 }\end{array}$
$\begin{array}{lllllll}\mathrm{N} & 13 & \mathrm{r} 20 & 11 & \mathrm{a} 20 & 10 & \mathrm{~d} 20\end{array}$
$\begin{array}{lllllll}\mathrm{O} & 13 & \mathrm{r} 21 & 11 & \mathrm{a} 21 & 10 & \mathrm{~d} 21\end{array}$
$\begin{array}{lllllll}\text { C } & 14 & \text { r22 } & 10 & \text { a22 } & 8 & \text { d } 22\end{array}$
$\begin{array}{lllllll}\mathrm{O} & 14 & \mathrm{r} 23 & 10 & \text { a23 } & 8 & \mathrm{~d} 23\end{array}$
$\begin{array}{lllllll}\text { C } & 20 & \text { r24 } & 13 & \text { a24 } & 11 & \text { d2 } 4\end{array}$
$\begin{array}{lllllll}\mathrm{H} & 11 & \mathrm{r} 25 & 10 & \mathrm{a} 25 & 8 & \mathrm{~d} 25\end{array}$
$\begin{array}{lllllll}\mathrm{H} & 8 & \mathrm{r} 26 & 7 & \mathrm{a} 26 & 3 & \mathrm{~d} 26\end{array}$
$\begin{array}{lllllll}\mathrm{H} & 1 & \mathrm{r} 27 & 2 & \mathrm{a} 27 & 3 & \mathrm{~d} 27\end{array}$
$\begin{array}{lllllll}\mathrm{H} & 4 & \mathrm{r} 28 & 3 & \mathrm{a} 28 & 2 & \mathrm{~d} 28\end{array}$
$\begin{array}{lllllll}\mathrm{H} & 5 & \mathrm{r} 29 & 4 & \mathrm{a} 29 & 3 & \mathrm{~d} 29\end{array}$
$\begin{array}{lllllll}\mathrm{H} & 6 & \text { r30 } & 5 & \text { a330 } & 4 & \text { d } 30\end{array}$
$\begin{array}{lllllll}\mathrm{H} & 9 & \text { r31 } & 2 & \text { a31 } & 1 & \text { d } 31\end{array}$
$\begin{array}{lllllll}\mathrm{H} & 12 & \text { r32 } & 7 & \text { a32 } & 3 & \text { d } 32\end{array}$
$\begin{array}{lllllll}\mathrm{H} & 12 & \text { r33 } & 7 & \text { a33 } & 3 & \text { d33 }\end{array}$
$\begin{array}{lllllll}\mathrm{H} & 15 & \mathrm{r} 34 & 7 & \mathrm{a} 34 & 3 & \mathrm{~d} 34\end{array}$

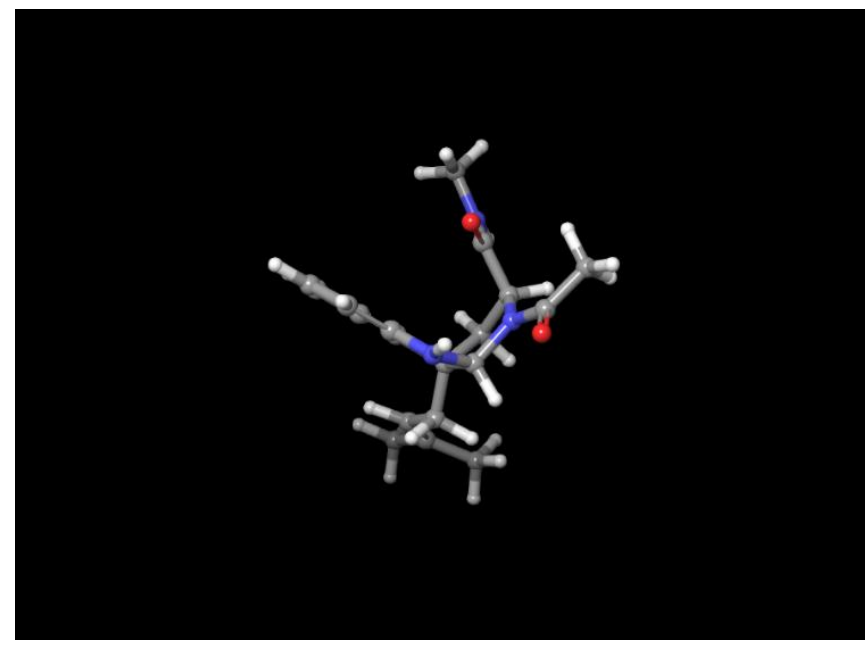



$\begin{array}{lllllll}\mathrm{H} & 15 & \mathrm{r} 35 & 7 & \mathrm{a} 35 & 3 & \mathrm{~d} 35\end{array}$
$\begin{array}{lllllll}\mathrm{H} & 16 & \mathrm{r} 36 & 15 & \text { a36 } & 7 & \mathrm{~d} 36\end{array}$
$\begin{array}{lllllll}\mathrm{H} & 18 & \mathrm{r} 37 & 17 & \mathrm{a} 37 & 16 & \mathrm{~d} 37\end{array}$
$\begin{array}{lllllll}\mathrm{H} & 18 & \mathrm{r} 38 & 17 & \mathrm{a} 38 & 16 & \mathrm{~d} 38\end{array}$
$\begin{array}{lllllll}\mathrm{H} & 18 & \mathrm{r} 39 & 17 & \mathrm{a} 39 & 16 & \mathrm{~d} 39\end{array}$
$\begin{array}{lllllll}\mathrm{H} & 19 & \mathrm{r} 40 & 17 & \mathrm{a} 40 & 16 & \mathrm{~d} 40\end{array}$
$\begin{array}{lllllll}\mathrm{H} & 19 & \mathrm{r} 41 & 17 & \mathrm{a} 41 & 16 & \mathrm{~d} 41\end{array}$
$\begin{array}{lllllll}\mathrm{H} & 19 & \mathrm{r} 42 & 17 & \mathrm{a} 42 & 16 & \mathrm{~d} 42\end{array}$
$\begin{array}{lllllll}\mathrm{H} & 20 & \mathrm{r} 43 & 13 & \mathrm{a} 43 & 11 & \mathrm{~d} 43\end{array}$
$\begin{array}{lllllll}\mathrm{H} & 22 & \mathrm{r} 44 & 14 & \mathrm{a} 44 & 10 & \mathrm{~d} 44\end{array}$
$\begin{array}{lllllll}\mathrm{H} & 22 & \mathrm{r} 45 & 14 & \mathrm{a} 45 & 10 & \mathrm{~d} 45\end{array}$
$\begin{array}{lllllll}\mathrm{H} & 22 & \mathrm{r} 46 & 14 & \mathrm{a} 46 & 10 & \mathrm{~d} 46\end{array}$
$\begin{array}{lllllll}\mathrm{H} & 24 & \mathrm{r} 47 & 20 & \mathrm{a} 47 & 13 & \mathrm{~d} 47\end{array}$
$\begin{array}{lllllll}\mathrm{H} & 24 & \mathrm{r} 48 & 20 & \mathrm{a} 48 & 13 & \mathrm{~d} 48\end{array}$
$\begin{array}{lllllll}\mathrm{H} & 24 & \mathrm{r} 49 & 20 & \mathrm{a} 49 & 13 & \mathrm{~d} 49\end{array}$
Variables:

$$
\begin{aligned}
& \mathrm{r} 2=1.3993 \\
& \mathrm{r} 3=1.4119 \\
& \mathrm{a} 3=120.97 \\
& \mathrm{r} 4=1.3911 \\
& \mathrm{a} 4=120.05 \\
& \mathrm{~d} 4=0.66 \\
& \mathrm{r} 5=1.4069 \\
& \mathrm{a} 5=119.53 \\
& \mathrm{~d} 5=359.47 \\
& \mathrm{r} 6=1.3995 \\
& \mathrm{a} 6=119.91 \\
& \mathrm{~d} 6=0.05 \\
& \mathrm{r} 7=1.5297 \\
& \mathrm{a} 7=108.86 \\
& \mathrm{~d} 7=181.00 \\
& \mathrm{r} 8=1.5620 \\
& \mathrm{a} 8=100.95 \\
& \mathrm{~d} 8=346.68 \\
& \mathrm{r} 9=1.3872 \\
& \mathrm{a} 9=128.78 \\
& \mathrm{~d} 9=181.59
\end{aligned}
$$




$$
\begin{aligned}
& \mathrm{r} 10=1.4763 \\
& \mathrm{a} 10=104.20 \\
& \mathrm{~d} 10=260.22 \\
& \mathrm{r} 11=1.4556 \\
& \mathrm{a} 11=113.59 \\
& \mathrm{~d} 11=0.83 \\
& \mathrm{r} 12=1.5419 \\
& \mathrm{a} 12=114.00 \\
& \mathrm{~d} 12=235.81 \\
& \mathrm{r} 13=1.5525 \\
& \mathrm{a} 13=112.01 \\
& \mathrm{~d} 13=104.58 \\
& \mathrm{r} 14=1.3654 \\
& \mathrm{a} 14=119.15 \\
& \mathrm{~d} 14=179.80 \\
& \mathrm{r} 15=1.5550 \\
& \mathrm{a} 15=112.76 \\
& \mathrm{~d} 15=105.14 \\
& \mathrm{r} 16=1.5038 \\
& \mathrm{a} 16=115.20 \\
& \mathrm{~d} 16=67.12 \\
& \mathrm{r} 21=1.5151 \\
& \mathrm{r} 17=1.3479 \\
& \mathrm{a} 17=128.23 \\
& \mathrm{~d} 17=115.18 \\
& \mathrm{r} 18=1.5100 \\
& \mathrm{a} 18=120.65 \\
& \mathrm{~d} 18=179.11 \\
& \mathrm{r} 19=1.5086 \\
& \mathrm{a} 19=125.09 \\
& \mathrm{~d} 19=359.58 \\
& \mathrm{r} 20=1.3547 \\
& \mathrm{~d} 20
\end{aligned}
$$




$$
\begin{aligned}
& \mathrm{a} 22=117.82 \\
& \mathrm{~d} 22=176.12 \\
& \mathrm{r} 23=1.2315 \\
& \mathrm{a} 23=120.25 \\
& \mathrm{~d} 23=356.91 \\
& \mathrm{r} 24=1.4505 \\
& \mathrm{a} 24=123.51 \\
& \mathrm{~d} 24=182.58 \\
& \mathrm{r} 25=1.1024 \\
& \mathrm{a} 25=110.66 \\
& \mathrm{~d} 25=222.87 \\
& \text { r26 }=1.1003 \\
& \mathrm{a} 26=114.11 \\
& \mathrm{~d} 26=142.27 \\
& \text { r27 }=1.0926 \\
& \mathrm{a} 27=120.81 \\
& \mathrm{~d} 27=180.35 \\
& \text { r28 }=1.0930 \\
& \text { a28 }=120.68 \\
& \mathrm{~d} 28=178.73 \\
& \text { r29 }=1.0925 \\
& \text { a29 }=119.95 \\
& \mathrm{~d} 29=179.77 \\
& \text { r30 }=1.0937 \\
& \mathrm{a} 30=119.63 \\
& \mathrm{~d} 30=179.77 \\
& \text { r31 }=1.0146 \\
& \text { a31 }=121.27 \\
& \mathrm{~d} 31=334.57 \\
& \text { r32 }=1.1008 \\
& \mathrm{a} 32=109.79 \\
& \mathrm{~d} 32=195.44 \\
& \text { r33 }=1.0981 \\
& \mathrm{a} 33=112.36 \\
& \mathrm{~d} 33=314.82 \\
& \text { r34= } 1.1007 \\
& \text { a34= } 107.69
\end{aligned}
$$




$$
\begin{aligned}
& \mathrm{d} 34=191.53 \\
& \text { r35 }=1.1049 \\
& \text { a } 35=107.11 \\
& \mathrm{~d} 35=305.62 \\
& \text { r36 }=1.0963 \\
& \text { a36 }=114.95 \\
& \mathrm{~d} 36=294.13 \\
& \text { r37 }=1.1048 \\
& \text { a37 }=110.95 \\
& \mathrm{~d} 37=121.89 \\
& \text { r38 }=1.1049 \\
& \mathrm{a} 38=110.93 \\
& \mathrm{~d} 38=239.59 \\
& \text { r39 }=1.1001 \\
& \text { a39 }=112.16 \\
& \mathrm{~d} 39=0.71 \\
& \mathrm{r} 40=1.1044 \\
& \mathrm{a} 40=110.45 \\
& \mathrm{~d} 40=238.95 \\
& \mathrm{r} 41=1.0980 \\
& \mathrm{a} 41=113.71 \\
& \mathrm{~d} 41=\quad 0.42 \\
& \mathrm{r} 42=1.1045 \\
& \mathrm{a} 42=110.43 \\
& \mathrm{~d} 42=121.89 \\
& \mathrm{r} 43=1.0137 \\
& \mathrm{a} 43=118.78 \\
& \mathrm{~d} 43=\quad 1.11 \\
& \mathrm{r} 44=1.0968 \\
& \text { a } 44=108.36 \\
& \mathrm{~d} 44=184.03 \\
& \mathrm{r} 45=1.1014 \\
& \mathrm{a} 45=111.54 \\
& \mathrm{~d} 45=304.26 \\
& \mathrm{r} 46=1.0997 \\
& \mathrm{a} 46=110.29 \\
& \mathrm{~d} 46=64.24
\end{aligned}
$$


$\mathrm{r} 47=1.1020$

$\mathrm{a} 47=111.08$

$\mathrm{d} 47=120.90$

$\mathrm{r} 48=1.1021$

$\mathrm{a} 48=111.27$

$\mathrm{d} 48=241.86$

$\mathrm{r} 49=1.0969$

$\mathrm{a} 49=108.17$

$\mathrm{d} 49=\quad 1.38$ 


\section{SRR20}
$\begin{array}{ll}0 & 1\end{array}$
C
C 11 r2
C $2 \begin{array}{llll}\text { r } 3 & 1 & \text { a3 }\end{array}$
$\begin{array}{lllllll}\text { C } & 3 & \text { r4 } & 2 & \text { a } 4 & 1 & \mathrm{~d} 4\end{array}$
$\begin{array}{lllllll}\mathrm{C} & 4 & \mathrm{r} 5 & 3 & \mathrm{a} 5 & 2 & \mathrm{~d} 5\end{array}$
C $5 \begin{array}{llllll}5 & \mathrm{r} 6 & 4 & \mathrm{a} 6 & 3 & \mathrm{~d} 6\end{array}$
$\begin{array}{lllllll}\text { C } & 3 & \text { r7 } & 2 & \text { a7 } & 1 & \text { d7 }\end{array}$
$\begin{array}{lllllll}\mathrm{C} & 7 & \mathrm{r} 8 & 3 & \mathrm{a} 8 & 2 & \mathrm{~d} 8\end{array}$
$\begin{array}{lllllll}\mathrm{N} & 2 & \mathrm{r} 9 & 1 & \mathrm{a} 9 & 3 & \mathrm{~d} 9\end{array}$
$\begin{array}{lllllll}\mathrm{N} & 8 & \mathrm{r} 10 & 7 & \mathrm{a} 10 & 3 & \mathrm{~d} 10\end{array}$
$\begin{array}{lllllll}\text { C } & 10 & \text { r11 } & 8 & \text { a11 } & 7 & \text { d11 }\end{array}$
$\begin{array}{lllllll}\text { C } & 11 & \text { r12 } & 10 & \text { a12 } & 8 & \text { d12 }\end{array}$
$\begin{array}{lllllll}\text { C } & 11 & \text { r13 } & 10 & \text { a13 } & 8 & \text { d } 13\end{array}$
$\begin{array}{lllllll}\text { C } & 10 & \text { r14 } & 8 & \text { a14 } & 7 & \text { d14 }\end{array}$
$\begin{array}{lllllll}\text { C } & 7 & \text { r15 } & 3 & \text { a15 } & 2 & \text { d } 15\end{array}$
$\begin{array}{lllllll}\mathrm{C} & 15 & \mathrm{r} 16 & 7 & \mathrm{a} 16 & 3 & \mathrm{~d} 16\end{array}$
$\begin{array}{lllllll}\text { C } & 16 & \text { r17 } & 15 & \text { a17 } & 7 & \text { d17 }\end{array}$
$\begin{array}{lllllll}\text { C } & 17 & \text { r18 } & 16 & \text { a18 } & 15 & \text { d18 }\end{array}$
$\begin{array}{lllllll}\text { C } & 17 & \text { r19 } & 16 & \text { a19 } & 15 & \text { d19 }\end{array}$
$\begin{array}{lllllll}\mathrm{N} & 13 & \mathrm{r} 20 & 11 & \mathrm{a} 20 & 10 & \mathrm{~d} 20\end{array}$
$\begin{array}{lllllll}\mathrm{O} & 13 & \mathrm{r} 21 & 11 & \mathrm{a} 21 & 10 & \mathrm{~d} 21\end{array}$
$\begin{array}{lllllll}\text { C } & 14 & \text { r22 } & 10 & \text { a22 } & 8 & \text { d } 22\end{array}$
$\begin{array}{lllllll}\text { O } & 14 & \text { r23 } & 10 & \text { a23 } & 8 & \text { d23 }\end{array}$
$\begin{array}{lllllll}\text { C } & 20 & \text { r24 } & 13 & \text { a24 } & 11 & \text { d24 }\end{array}$
$\begin{array}{lllllll}\mathrm{H} & 11 & \mathrm{r} 25 & 10 & \mathrm{a} 25 & 8 & \mathrm{~d} 25\end{array}$
$\begin{array}{lllllll}\mathrm{H} & 8 & \mathrm{r} 26 & 7 & \mathrm{a} 26 & 3 & \mathrm{~d} 26\end{array}$
$\begin{array}{lllllll}\mathrm{H} & 1 & \mathrm{r} 27 & 2 & \mathrm{a} 27 & 3 & \mathrm{~d} 27\end{array}$
$\begin{array}{lllllll}\mathrm{H} & 4 & \mathrm{r} 28 & 3 & \mathrm{a} 28 & 2 & \mathrm{~d} 28\end{array}$
$\begin{array}{lllllll}\mathrm{H} & 5 & \mathrm{r} 29 & 4 & \mathrm{a} 29 & 3 & \mathrm{~d} 29\end{array}$
$\begin{array}{lllllll}\mathrm{H} & 6 & \mathrm{r} 30 & 5 & \text { a330 } & 4 & \mathrm{~d} 30\end{array}$
$\begin{array}{lllllll}\mathrm{H} & 9 & \mathrm{r} 31 & 2 & \mathrm{a} 31 & 1 & \mathrm{~d} 31\end{array}$
$\begin{array}{lllllll}\mathrm{H} & 12 & \text { r32 } & 11 & \text { a32 } & 10 & \text { d } 32\end{array}$
$\begin{array}{lllllll}\mathrm{H} & 12 & \text { r33 } & 11 & \text { a33 } & 10 & \text { d } 33\end{array}$
$\begin{array}{lllllll}\mathrm{H} & 15 & \mathrm{r} 34 & 7 & \mathrm{a} 34 & 3 & \mathrm{~d} 34\end{array}$ 


$\begin{array}{lllllll}\mathrm{H} & 15 & \mathrm{r} 35 & 7 & \mathrm{a} 35 & 3 & \mathrm{~d} 35 \\ \mathrm{H} & 16 & \mathrm{r} 36 & 15 & \mathrm{a} 36 & 7 & \mathrm{~d} 36 \\ \mathrm{H} & 18 & \mathrm{r} 37 & 17 & \mathrm{a} 37 & 16 & \mathrm{~d} 37 \\ \mathrm{H} & 18 & \mathrm{r} 38 & 17 & \mathrm{a} 38 & 16 & \mathrm{~d} 38 \\ \mathrm{H} & 18 & \mathrm{r} 39 & 17 & \mathrm{a} 39 & 16 & \mathrm{~d} 39 \\ \mathrm{H} & 19 & \mathrm{r} 40 & 17 & \mathrm{a} 40 & 16 & \mathrm{~d} 40 \\ \mathrm{H} & 19 & \mathrm{r} 41 & 17 & \mathrm{a} 41 & 16 & \mathrm{~d} 41 \\ \mathrm{H} & 19 & \mathrm{r} 42 & 17 & \mathrm{a} 42 & 16 & \mathrm{~d} 42 \\ \mathrm{H} & 20 & \mathrm{r} 43 & 13 & \mathrm{a} 43 & 11 & \mathrm{~d} 43 \\ \mathrm{H} & 22 & \mathrm{r} 44 & 14 & \mathrm{a} 44 & 10 & \mathrm{~d} 44 \\ \mathrm{H} & 22 & \mathrm{r} 45 & 14 & \mathrm{a} 45 & 10 & \mathrm{~d} 45 \\ \mathrm{H} & 22 & \mathrm{r} 46 & 14 & \mathrm{a} 46 & 10 & \mathrm{~d} 46 \\ \mathrm{H} & 24 & \mathrm{r} 47 & 20 & \mathrm{a} 47 & 13 & \mathrm{~d} 47 \\ \mathrm{H} & 24 & \mathrm{r} 48 & 20 & \mathrm{a} 48 & 13 & \mathrm{~d} 48 \\ \mathrm{H} & 24 & \mathrm{r} 49 & 20 & \mathrm{a} 49 & 13 & \mathrm{~d} 49 \\ \text { Variables: } & & & & \end{array}$

$\mathrm{r} 2=1.3999$

$\mathrm{r} 3=1.4071$

$\mathrm{a} 3=121.10$

$\mathrm{r} 4=1.3893$

$\mathrm{a} 4=120.17$

$\mathrm{d} 4=359.13$

r5 $=1.4058$

$\mathrm{a} 5=119.48$

$\mathrm{d} 5=0.50$

r6 $=1.4001$

$\mathrm{a} 6=119.91$

$\mathrm{d} 6=0.07$

$\mathrm{r} 7=1.5248$

$\mathrm{a} 7=109.42$

$\mathrm{d} 7=180.41$

$\mathrm{r} 8=1.5724$

$\mathrm{a} 8=101.89$

$\mathrm{d} 8=351.24$

$\mathrm{r} 9=1.3881$

$\mathrm{a} 9=128.74$

$\mathrm{d} 9=182.29$ 


$$
\begin{aligned}
& \mathrm{r} 10=1.4777 \\
& \mathrm{a} 10=104.84 \\
& \mathrm{~d} 10=253.58 \\
& \mathrm{r} 11=1.4754 \\
& \mathrm{a} 11=113.12 \\
& \mathrm{~d} 11=4.92 \\
& \mathrm{r} 12=1.5293 \\
& \mathrm{a} 12=103.55 \\
& \mathrm{~d} 12=339.86 \\
& \mathrm{r} 13=1.5526 \\
& \mathrm{a} 13=114.61 \\
& \mathrm{~d} 13=104.53 \\
& \mathrm{r} 14=1.3634 \\
& \mathrm{a} 14=124.93 \\
& \mathrm{~d} 14=196.17 \\
& \mathrm{r} 15=1.5541 \\
& \mathrm{a} 15=112.12 \\
& \mathrm{~d} 15=112.65 \\
& \mathrm{r} 16=1.5038 \\
& \mathrm{a} 16=115.49 \\
& \mathrm{~d} 16=304.81 \\
& \mathrm{r} 17=1.3471 \\
& \mathrm{a} 17=128.35 \\
& \mathrm{~d} 17=120.83 \\
& \mathrm{r} 18=1.5099 \\
& \mathrm{a} 18=120.70 \\
& \mathrm{~d} 18=179.04 \\
& \mathrm{r} 19=1.5085 \\
& \mathrm{a} 19=124.97 \\
& \mathrm{~d} 19=359.29 \\
& \mathrm{r} 20=1.3516 \\
& \mathrm{~d} 20.76
\end{aligned}
$$




$$
\begin{aligned}
& \mathrm{a} 22=118.09 \\
& \mathrm{~d} 22=\quad 0.61 \\
& \mathrm{r} 23=1.2352 \\
& \mathrm{a} 23=121.03 \\
& \mathrm{~d} 23=180.19 \\
& \text { r24= } 1.4479 \\
& \text { a24= } 123.46 \\
& \mathrm{~d} 24=178.29 \\
& \text { r25 }=1.1019 \\
& \text { a25 }=107.46 \\
& \mathrm{~d} 25=221.68 \\
& \text { r26 }=1.0993 \\
& \mathrm{a} 26=112.99 \\
& \mathrm{~d} 26=135.64 \\
& \text { r27 }=1.0927 \\
& \mathrm{a} 27=120.97 \\
& \mathrm{~d} 27=180.52 \\
& \text { r28 }=1.0938 \\
& \mathrm{a} 28=120.51 \\
& \mathrm{~d} 28=179.88 \\
& \text { r29 }=1.0925 \\
& \text { a29= } 120.02 \\
& \mathrm{~d} 29=179.69 \\
& \text { r30 }=1.0936 \\
& \text { a30 }=119.67 \\
& \mathrm{~d} 30=179.59 \\
& \text { r31 }=1.0130 \\
& \text { a31 }=120.82 \\
& \mathrm{~d} 31=339.58 \\
& \text { r32 }=1.1003 \\
& \text { a32 }=109.16 \\
& \mathrm{~d} 32=268.99 \\
& \text { r33 }=1.0968 \\
& \text { a33 }=111.00 \\
& \mathrm{~d} 33=149.44 \\
& \text { r34 }=1.1004 \\
& \text { a34 }=107.56
\end{aligned}
$$




$$
\begin{aligned}
& \mathrm{d} 34=69.64 \\
& \text { r35 }=1.1064 \\
& \text { a35 }=106.98 \\
& \text { d } 35=183.28 \\
& \text { r36 }=1.0974 \\
& \text { a36 }=114.94 \\
& \text { d } 36=300.05 \\
& \text { r37 }=1.1048 \\
& \mathrm{a} 37=110.91 \\
& \mathrm{~d} 37=121.49 \\
& \text { r38 }=1.1049 \\
& \text { a38 }=110.98 \\
& \mathrm{~d} 38=239.22 \\
& \text { r39 }=1.1003 \\
& \mathrm{a} 39=112.15 \\
& \mathrm{~d} 39=\quad 0.37 \\
& \mathrm{r} 40=1.1044 \\
& \mathrm{a} 40=110.38 \\
& \mathrm{~d} 40=238.33 \\
& \mathrm{r} 41=1.0977 \\
& \mathrm{a} 41=113.67 \\
& \mathrm{~d} 41=359.67 \\
& \mathrm{r} 42=1.1046 \\
& \mathrm{a} 42=110.49 \\
& \mathrm{~d} 42=121.21 \\
& \mathrm{r} 43=1.0228 \\
& \mathrm{a} 43=116.66 \\
& \mathrm{~d} 43=\quad 3.04 \\
& \text { r44 }=1.0964 \\
& \mathrm{a} 44=108.02 \\
& \mathrm{~d} 44=180.43 \\
& \mathrm{r} 45=1.1002 \\
& \mathrm{a} 45=112.10 \\
& \mathrm{~d} 45=300.14 \\
& \mathrm{r} 46=1.1011 \\
& \mathrm{a} 46=111.30 \\
& \mathrm{~d} 46=60.63
\end{aligned}
$$


$\mathrm{r} 47=1.1035$

$\mathrm{a} 47=111.58$

$\mathrm{d} 47=247.93$

$\mathrm{r} 48=1.0973$

$\mathrm{a} 48=108.64$

$\mathrm{d} 48=7.52$

$\mathrm{r} 49=1.1022$

$\mathrm{a} 49=110.98$

$\mathrm{d} 49=127.28$ 


\section{SRR22}
$\begin{array}{ll}0 & 1\end{array}$
$\mathrm{C}$
C $\quad 1 \quad$ r2
C $2 \begin{array}{llll}\text { r } 3 & 1 & \text { a3 }\end{array}$
$\begin{array}{lllllll}\mathrm{C} & 3 & \mathrm{r} 4 & 2 & \mathrm{a} 4 & 1 & \mathrm{~d} 4\end{array}$
$\begin{array}{lllllll}\mathrm{C} & 4 & \mathrm{r} 5 & 3 & \mathrm{a} 5 & 2 & \mathrm{~d} 5\end{array}$
$\begin{array}{lllllll}\mathrm{C} & 5 & \mathrm{r} 6 & 4 & \mathrm{a} 6 & 3 & \mathrm{~d} 6\end{array}$
$\begin{array}{lllllll}\mathrm{C} & 3 & \mathrm{r} 7 & 2 & \mathrm{a} 7 & 1 & \mathrm{~d} 7\end{array}$
$\begin{array}{lllllll}\mathrm{C} & 7 & \mathrm{r} 8 & 3 & \mathrm{a} 8 & 2 & \mathrm{~d} 8\end{array}$
$\begin{array}{lllllll}\mathrm{N} & 2 & \mathrm{r} 9 & 1 & \mathrm{a} 9 & 3 & \mathrm{~d} 9\end{array}$
$\begin{array}{lllllll}\mathrm{N} & 8 & \mathrm{r} 10 & 7 & \mathrm{a} 10 & 3 & \mathrm{~d} 10\end{array}$
$\begin{array}{lllllll}\text { C } & 10 & \text { r11 } & 8 & \text { a11 } & 7 & \text { d11 }\end{array}$
$\begin{array}{lllllll}\text { C } & 7 & \text { r12 } & 3 & \text { a12 } & 2 & \text { d12 }\end{array}$
$\begin{array}{lllllll}\text { C } & 11 & \text { r13 } & 10 & \text { a13 } & 8 & \text { d13 }\end{array}$
$\begin{array}{lllllll}\text { C } & 10 & \text { r14 } & 8 & \text { a14 } & 7 & \text { d14 }\end{array}$
$\begin{array}{lllllll}\text { C } & 7 & \text { r15 } & 3 & \text { a15 } & 2 & \text { d15 }\end{array}$
$\begin{array}{lllllll}\text { C } & 15 & \text { r16 } & 7 & \text { a16 } & 3 & \text { d16 }\end{array}$
$\begin{array}{lllllll}\mathrm{C} & 16 & \mathrm{r} 17 & 15 & \text { a17 } & 7 & \mathrm{~d} 17\end{array}$
$\begin{array}{lllllll}\text { C } & 17 & \text { r18 } & 16 & \text { a18 } & 15 & \text { d18 }\end{array}$
$\begin{array}{lllllll}\mathrm{C} & 17 & \mathrm{r} 19 & 16 & \mathrm{a} 19 & 15 & \mathrm{~d} 19\end{array}$
$\begin{array}{lllllll}\mathrm{N} & 13 & \mathrm{r} 20 & 11 & \mathrm{a} 20 & 10 & \mathrm{~d} 20\end{array}$
$\begin{array}{lllllll}\mathrm{O} & 13 & \mathrm{r} 21 & 11 & \mathrm{a} 21 & 10 & \mathrm{~d} 21\end{array}$
$\begin{array}{lllllll}\mathrm{C} & 14 & \mathrm{r} 22 & 10 & \mathrm{a} 22 & 8 & \mathrm{~d} 22\end{array}$
$\begin{array}{lllllll}\mathrm{O} & 14 & \mathrm{r} 23 & 10 & \mathrm{a} 23 & 8 & \mathrm{~d} 23\end{array}$
$\begin{array}{lllllll}\text { C } & 20 & \text { r24 } & 13 & \text { a24 } & 11 & \text { d24 }\end{array}$
$\begin{array}{lllllll}\mathrm{H} & 11 & \mathrm{r} 25 & 10 & \mathrm{a} 25 & 8 & \mathrm{~d} 25\end{array}$
$\begin{array}{lllllll}\mathrm{H} & 8 & \mathrm{r} 26 & 7 & \mathrm{a} 26 & 3 & \mathrm{~d} 26\end{array}$
$\begin{array}{lllllll}\mathrm{H} & 1 & \mathrm{r} 27 & 2 & \mathrm{a} 27 & 3 & \mathrm{~d} 27\end{array}$
$\begin{array}{lllllll}\mathrm{H} & 4 & \mathrm{r} 28 & 3 & \mathrm{a} 28 & 2 & \mathrm{~d} 28\end{array}$
$\begin{array}{lllllll}\mathrm{H} & 5 & \mathrm{r} 29 & 4 & \mathrm{a} 29 & 3 & \mathrm{~d} 29\end{array}$
$\begin{array}{lllllll}\mathrm{H} & 6 & \mathrm{r} 30 & 5 & \mathrm{a} 30 & 4 & \mathrm{~d} 30\end{array}$
$\begin{array}{lllllll}\mathrm{H} & 9 & \mathrm{r} 31 & 2 & \mathrm{a} 31 & 1 & \mathrm{~d} 31\end{array}$
$\begin{array}{lllllll}\mathrm{H} & 12 & \text { r32 } & 7 & \text { a32 } & 3 & \text { d } 32\end{array}$
$\begin{array}{lllllll}\mathrm{H} & 12 & \text { r33 } & 7 & \text { a33 } & 3 & \text { d } 33\end{array}$
$\begin{array}{lllllll}\mathrm{H} & 15 & \mathrm{r} 34 & 7 & \mathrm{a} 34 & 3 & \mathrm{~d} 34\end{array}$

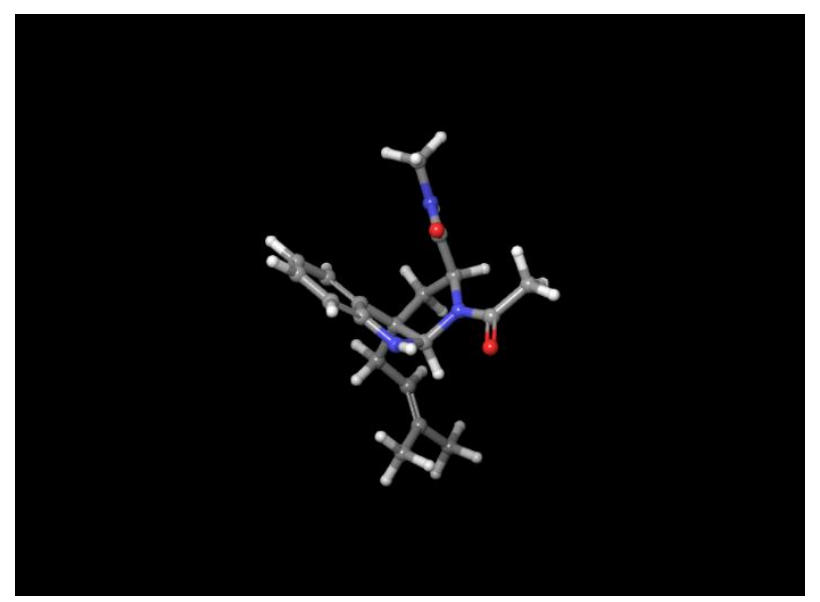




$\begin{array}{lllllll}\mathrm{H} & 15 & \mathrm{r} 35 & 7 & \mathrm{a} 35 & 3 & \mathrm{~d} 35 \\ \mathrm{H} & 16 & \mathrm{r} 36 & 15 & \mathrm{a} 36 & 7 & \mathrm{~d} 36 \\ \mathrm{H} & 18 & \mathrm{r} 37 & 17 & \mathrm{a} 37 & 16 & \mathrm{~d} 37 \\ \mathrm{H} & 18 & \mathrm{r} 38 & 17 & \mathrm{a} 38 & 16 & \mathrm{~d} 38 \\ \mathrm{H} & 18 & \mathrm{r} 39 & 17 & \mathrm{a} 39 & 16 & \mathrm{~d} 39 \\ \mathrm{H} & 19 & \mathrm{r} 40 & 17 & \mathrm{a} 40 & 16 & \mathrm{~d} 40 \\ \mathrm{H} & 19 & \mathrm{r} 41 & 17 & \mathrm{a} 41 & 16 & \mathrm{~d} 41 \\ \mathrm{H} & 19 & \mathrm{r} 42 & 17 & \mathrm{a} 42 & 16 & \mathrm{~d} 42 \\ \mathrm{H} & 20 & \mathrm{r} 43 & 13 & \mathrm{a} 43 & 11 & \mathrm{~d} 43 \\ \mathrm{H} & 22 & \mathrm{r} 44 & 14 & \mathrm{a} 44 & 10 & \mathrm{~d} 44 \\ \mathrm{H} & 22 & \mathrm{r} 45 & 14 & \mathrm{a} 45 & 10 & \mathrm{~d} 45 \\ \mathrm{H} & 22 & \mathrm{r} 46 & 14 & \mathrm{a} 46 & 10 & \mathrm{~d} 46 \\ \mathrm{H} & 24 & \mathrm{r} 47 & 20 & \mathrm{a} 47 & 13 & \mathrm{~d} 47 \\ \mathrm{H} & 24 & \mathrm{r} 48 & 20 & \mathrm{a} 48 & 13 & \mathrm{~d} 48 \\ \mathrm{H} & 24 & \mathrm{r} 49 & 20 & \mathrm{a} 49 & 13 & \mathrm{~d} 49 \\ \text { Variables: } & & & & \end{array}$

.

$\mathrm{r} 2=1.3996$

r3 $=1.4108$

$\mathrm{a} 3=120.79$

$\mathrm{r} 4=1.3900$

$\mathrm{a} 4=120.37$

$\mathrm{d} 4=359.29$

r $5=1.4067$

$\mathrm{a} 5=119.38$

$\mathrm{d} 5=0.69$

r6 $=1.4001$

$\mathrm{a} 6=119.86$

$\mathrm{d} 6=359.70$

$\mathrm{r} 7=1.5276$

$\mathrm{a} 7=108.98$

$\mathrm{d} 7=181.52$

$\mathrm{r} 8=1.5602$

$\mathrm{a} 8=101.07$

$\mathrm{d} 8=346.86$

$\mathrm{r} 9=1.3875$

$\mathrm{a} 9=129.07$

$\mathrm{d} 9=182.23$ 


$$
\begin{aligned}
& \mathrm{r} 10=1.4765 \\
& \mathrm{a} 10=104.14 \\
& \mathrm{~d} 10=260.16 \\
& \mathrm{r} 11=1.4557 \\
& \mathrm{a} 11=113.65 \\
& \mathrm{~d} 11=1.28 \\
& \mathrm{r} 12=1.5422 \\
& \mathrm{a} 12=113.76 \\
& \mathrm{~d} 12=235.91 \\
& \mathrm{r} 13=1.5525 \\
& \mathrm{a} 13=111.98 \\
& \mathrm{~d} 13=104.32 \\
& \mathrm{r} 14=1.3652 \\
& \mathrm{a} 14=119.11 \\
& \mathrm{~d} 14=180.14 \\
& \mathrm{r} 15=1.5576 \\
& \mathrm{a} 15=109.74 \\
& \mathrm{~d} 15=106.88 \\
& \mathrm{r} 16=1.5058 \\
& \mathrm{a} 16=116.22 \\
& \mathrm{~d} 16=185.71 \\
& \mathrm{r} 21=1.5151 \\
& \mathrm{r} 17=1.3484 \\
& \mathrm{a} 17=127.71 \\
& \mathrm{~d} 17=105.83 \\
& \mathrm{r} 18=1.5095 \\
& \mathrm{a} 18=120.81 \\
& \mathrm{~d} 18=178.72 \\
& \mathrm{r} 19=1.5088 \\
& \mathrm{a} 19=124.76 \\
& \mathrm{~d} 19=359.22 \\
& \mathrm{a} 20=1.3548 \\
& \mathrm{~d} 20.53
\end{aligned}
$$




$$
\begin{aligned}
& \mathrm{a} 22=117.80 \\
& \mathrm{~d} 22=176.09 \\
& \mathrm{r} 23=1.2316 \\
& \mathrm{a} 23=120.27 \\
& \mathrm{~d} 23=356.91 \\
& \mathrm{r} 24=1.4505 \\
& \mathrm{a} 24=123.55 \\
& \mathrm{~d} 24=182.69 \\
& \mathrm{r} 25=1.1027 \\
& \text { a } 25=110.65 \\
& \mathrm{~d} 25=222.49 \\
& \text { r26= } 1.0989 \\
& \mathrm{a} 26=114.28 \\
& \mathrm{~d} 26=142.28 \\
& \text { r27 }=1.0926 \\
& \mathrm{a} 27=120.86 \\
& \mathrm{~d} 27=180.55 \\
& \text { r28 }=1.0941 \\
& \text { a28 }=120.66 \\
& \mathrm{~d} 28=180.20 \\
& \text { r29 }=1.0925 \\
& \text { a29 }=120.03 \\
& \mathrm{~d} 29=179.54 \\
& \text { r30 }=1.0937 \\
& \mathrm{a} 30=119.58 \\
& \mathrm{~d} 30=179.71 \\
& \text { r31 }=1.0146 \\
& \mathrm{a} 31=121.38 \\
& \mathrm{~d} 31=334.04 \\
& \text { r32 }=1.0993 \\
& \mathrm{a} 32=110.19 \\
& \mathrm{~d} 32=195.22 \\
& \text { r33 }=1.0986 \\
& \mathrm{a} 33=112.04 \\
& \mathrm{~d} 33=314.92 \\
& \text { r34= } 1.0999 \\
& \text { a34= } 107.46
\end{aligned}
$$




$$
\begin{aligned}
& \mathrm{d} 34=310.61 \\
& \mathrm{r} 35=1.1041 \\
& \mathrm{a} 35=106.74 \\
& \mathrm{~d} 35=63.91 \\
& \mathrm{r} 36=1.0968 \\
& \mathrm{a} 36=115.58 \\
& \mathrm{~d} 36=284.76 \\
& \mathrm{r} 37=1.1046 \\
& \mathrm{a} 37=110.93 \\
& \mathrm{~d} 37=121.28 \\
& \mathrm{r} 38=1.1047 \\
& \mathrm{a} 38=110.87 \\
& \mathrm{~d} 38=238.98 \\
& \mathrm{r} 39=1.1001 \\
& \mathrm{a} 39=112.15 \\
& \mathrm{~d} 39= \\
& \mathrm{r} 40=1.1041 \\
& \mathrm{a} 40=110.29 \\
& \mathrm{~d} 46=110.30 \\
& \mathrm{~d} 40=235.51 \\
& \mathrm{r} 41=1.0979 \\
& \mathrm{a} 41=113.68 \\
& \mathrm{~d} 41=356.68 \\
& \mathrm{r} 42=1.1046 \\
& \mathrm{a} 42=110.52 \\
& \mathrm{~d} 42=118.47 \\
& \mathrm{r} 43=1.0138 \\
& \mathrm{a} 43=118.79 \\
& \mathrm{~d} 43= \\
& \mathrm{r} 44=1.0969 \\
& \mathrm{a} 44=108.37 \\
& \mathrm{~d} 44=183.88 \\
& \mathrm{a} 450
\end{aligned}
$$


$\mathrm{r} 47=1.1020$

$\mathrm{a} 47=111.18$

$\mathrm{d} 47=239.93$

$\mathrm{r} 48=1.0968$

$\mathrm{a} 48=108.17$

$\mathrm{d} 48=359.44$

$\mathrm{r} 49=1.1022$

$\mathrm{a} 49=111.18$

$\mathrm{d} 49=118.99$ 
High resolut on mass spectrum of motomabide (1)

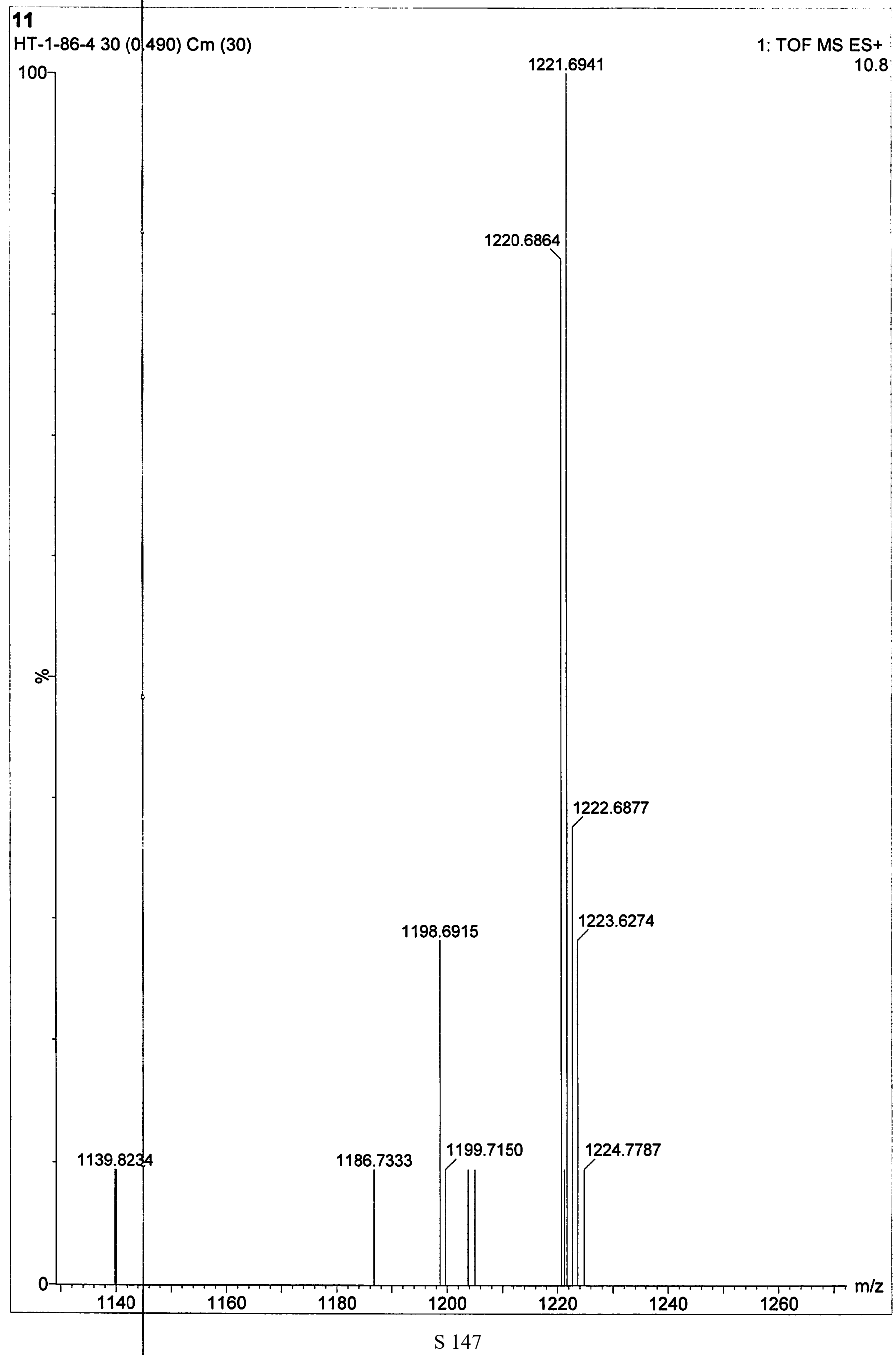

